\title{
15. UNDERWAY GEOPHYSICAL MEASUREMENTS OBTAINED ON THE GLOMAR CHALLENGER IN THE EASTERN NORTH ATLANTIC AND MEDITERRANEAN SEA
}

\author{
William B. F. Ryan, Lamont-Doherty Geological Observatory of Columbia University, Palisades, New York, \\ and \\ Ted B. Gustafson, Scripps Institution of Oceanography, University of California, La Jolla, California
}

\section{INTRODUCTION}

Routine geophysical measurements were obtained during Leg 13 of the Deep Sea Drilling Project while the Glomar Challenger steamed to and from the various drilling sites. The entire track from Lisbon, Portugal into the Mediterranean as far east as the Nile Cone, and then back again to Lisbon covered a distance of 4646 nautical miles. In fact, 38 per cent of the entire leg was spent underway, almost all of it in direct runs between the sites. Because of careful planning and excellent pre-site surveys, the drilling vessel was usually able to find the drilling targets with a minimum of time spent in surveys of her own.

The onboard instrumentation included a $12 \mathrm{kHz}$ precision echo-sounder, magnetometer, seismic reflection profiler, and a satellite navigation system. The instruments were maintained and operated by a technical staff of the Scripps Institution of Oceanography, in cooperation with the scientific party and the officers and crew of Global Marine, Inc.

\section{NAVIGATION}

The prime navigation system on the Glomar Challenger consisted of an I.T.T. Model 4007AB Satellite Navigation Receiver utilizing a PDP 8 digital computer and teletype display. Positions were obtained with this system through the entire 54-day leg, except for an occasional few hours of down time. Approximately 15 fixes were available each day when underway, and these were supplemented by celestial fixes and radar ranges and bearings when available or needed.

The ship's track ${ }^{1}$ was generated by interpolating deadreckoning readouts of course (gyro-compass headings) and speed (electromagnetic log) between the satellite "benchmarks" so as to position the location of all course and speed changes. The smoothed track was digitized, interval speeds and courses-made-good have been calculated, and the output is listed in Table 1. The track is displayed in a mosaic of sixteen mercator charts (Figure 1, A, B, C, etc.) at one half standard plotting sheet scale.

Not all sections of the track can be constructed with the same degree of accuracy. Whereas the positions of the satellite fixes themselves are generally good to \pm 0.2 mile, the intermediate positions between fixes can degrade to \pm 3 miles depending upon the length of time between fixes, and the amount of maneuvering of the vessel.

\footnotetext{
${ }^{1}$ The preliminary track was made by the bridge officers during the cruise. This track was subsequently edited and smoothed by the senior author, who assumes responsibility for any errors or omissions.
}

Details of the track in the vicinity of the individual sites are shown in maps appearing in the Site Chapters.

The dead-reckoning while locating the drilling target was facilitated with the deployment of surface buoys, and is presented in terms of relative positions without an implied absolute accuracy. The navigational control in the area of Site 121 in the Alboran Sea is particularly poor owing to strong and variable currents.

\section{Bathymetry}

Bathymetric profiles were obtained with a $12 \mathrm{kHz}$ echo-sounder using a Gifft Recorder and displaying on wet Mufax paper. Sweep rates were maintained at 1 second (400 tau) with a pulse width of $\approx 1$ millisecond. The echo-grams were digitized at all inflection points and the depth data have been corrected for the vessel's draft. Because the $12 \mathrm{kHz}$ system was inoperable from Lisbon to Site 121 , the depth data for this interval were obtained by digitizing the seismic reflection profiles with a consequent degradation in accuracy to \pm 5 tau.

\section{Magnetics}

Total intensity measurements of the earth's magnetic field were obtained with a Varian Proton Precession Magnetometer. The sensor unit was towed approximately 250 meters astern. The direct analog readings in gammas have been digitized and converted to residual anomalies using a spherical harmonic expression of the earth's regional magnetic field (Talwani, 1969).

\section{Topographic and Magnetic Profiles}

The digitized bathymetric, magnetic, and navigation data are graphically presented in Figure 2 . The navigation positions are annotated along the base of the plot (distance axis: one $\mathrm{cm}$ equal to twenty miles). Where the distance between fixes is large enough, interpolated headings and speeds are also shown. Date and time of day ${ }^{2}$ appear at the top of the plot along with annotations for even degree crossings of longitude and latitude. The depth units are in terms of two-way travel time ( 1 tau $=1 / 400$ th second $)$, and the topographic profile has a uniform exaggeration of 100 to 1 .

\section{Seismic Reflecting Profiling}

The reflection profiling system aboard the Challenger included: (1) a sound source of two Bolt $600 \mathrm{~A}$ pneumatic airguns (one 10 cubic inches, the other 30); (2) a listening array of twenty EVP 23 hydrophones, evenly spaced over

\footnotetext{
${ }^{2}$ Time-zone-1 was used throughout the leg.
} 
TABLE 1

Navigation Leg $13^{\text {a }}$

\begin{tabular}{|c|c|c|c|c|c|}
\hline Time & Latitude & Longitude & $\begin{array}{l}\text { Distance } \\
\text { (miles) }\end{array}$ & $\begin{array}{c}\text { Speed } \\
\text { (knots) }\end{array}$ & $\begin{array}{c}\text { Course } \\
\text { (degrees) }\end{array}$ \\
\hline
\end{tabular}

DEPART LISBON (August 14, 1970)

TABLE 1 - Continued

$\begin{array}{rllrlrrr}300 & 38^{\circ} 43.1^{\prime} \mathrm{N} & -9^{\circ} & 28.2^{\prime} \mathrm{W} & 0.0 & 10.6 & 216 \\ 800 & 38 & 00.0 & -10 & 07.5 & 53.0 & 11.1 & 215 \\ 1250 & 37 & 16.1 & -10 & 46.1 & 106.5 & 10.0 & 220 \\ 1330 & 37 & 11.0 & -10 & 51.5 & 113.2 & 11.5 & 228 \\ 1358 & 37 & 07.4 & -10 & 56.5 & 118.5 & 10.9 & 224 \\ 1635 & 36 & 46.8 & -11 & 21.3 & 147.1 & 7.4 & 261 \\ 1646 & 36 & 46.6 & -11 & 23.0 & 148.5 & 8.1 & 257 \\ 1705 & 36 & 46.0 & -11 & 26.1 & 151.0 & 7.4 & 171 \\ 1738 & 36 & 42.0 & -11 & 25.3 & 155.1 & 4.5 & 175 \\ 1750 & 36 & 41.1 & -11 & 25.2 & 156.0 & 2.8 & 329 \\ 1800 & 36 & 41.5 & -11 & 25.5 & 156.5 & 1.1 & 253 \\ 1820 & 36 & 41.4 & -11 & 25.9 & 156.8 & 0.0 & 270\end{array}$

\begin{tabular}{lcccc}
\hline Time Latitude & Longitude & $\begin{array}{c}\text { Distance } \\
\text { (miles) }\end{array}$ & $\begin{array}{c}\text { Speed } \\
\text { (knots) }\end{array}$ & $\begin{array}{c}\text { Course } \\
\text { (degrees) }\end{array}$ \\
\hline
\end{tabular}

(August 22, 1970)

$\begin{array}{rrrrllll}014 & 36 & 52.7 & -1 & 36.3 & 674.1 & 10.2 & 53 \\ 243 & 37 & 08.1 & -1 & 11.0 & 699.5 & 11.1 & 52 \\ 612 & 37 & 31.8 & -0 & 32.6 & 738.2 & 11.1 & 51 \\ 623 & 37 & 33.1 & -0 & 30.6 & 740.2 & 11.5 & 38 \\ 940 & 38 & 02.7 & -0 & 00.9 & 778.0 & 10.9 & 41 \\ 1304 & 38 & 30.4 & 0 & 30.2 \mathrm{E} & 814.9 & 10.7 & 43 \\ 1450 & 38 & 44.2 & 0 & 46.8 & 833.8 & 10.3 & 41 \\ 1724 & 39 & 04.3 & 1 & 08.9 & 860.4 & 10.2 & 43 \\ 1746 & 39 & 07.1 & 1 & 12.2 & 864.1 & 10.5 & 39 \\ 2254 & 39 & 49.3 & 1 & 56.0 & 918.2 & 10.3 & 39\end{array}$

SITE 120 (August 17, 1970)

$\begin{array}{rrrrrrrr}312 & 36 & 41.4 & -11 & 25.9 & 156.8 & 3.5 & 286 \\ 338 & 36 & 41.8 & -11 & 27.7 & 158.3 & 6.6 & 122 \\ 344 & 36 & 41.4 & -11 & 27.0 & 159.0 & 5.9 & 94 \\ 353 & 36 & 41.4 & -11 & 25.9 & 159.9 & 10.5 & 102 \\ 714 & 36 & 33.9 & -10 & 43.2 & 195.0 & 10.4 & 101 \\ 1004 & 36 & 28.5 & -10 & 07.2 & 224.5 & 10.1 & 100 \\ 1152 & 36 & 25.4 & -9 & 44.9 & 242.7 & 10.1 & 100 \\ 1406 & 36 & 21.5 & -9 & 17.4 & 265.1 & 10.4 & 101 \\ 1552 & 36 & 18.0 & -8 & 55.0 & 283.5 & 10.8 & 103 \\ 1756 & 36 & 13.0 & -8 & 28.0 & 305.8 & 10.8 & 101 \\ 2202 & 36 & 04.6 & -7 & 34.3 & 350.0 & 10.7 & 101\end{array}$

(August 23, 1970)

$\begin{array}{rrrrrrrr}248 & 40 & 20.6 & 2 & 28.6 & 958.2 & 9.2 & 39 \\ 309 & 40 & 23.1 & 2 & 31.2 & 961.4 & 9.8 & 46 \\ 336 & 40 & 26.2 & 2 & 35.4 & 965.8 & 9.7 & 59 \\ 340 & 40 & 26.5 & 2 & 36.1 & 966.5 & 9.7 & 62 \\ 357 & 40 & 27.8 & 2 & 39.3 & 969.2 & 3.9 & 64 \\ 412 & 40 & 28.2 & 2 & 40.4 & 970.2 & 3.0 & 171 \\ 417 & 40 & 28.0 & 2 & 40.5 & 970.4 & 9.0 & 244 \\ 433 & 40 & 26.9 & 2 & 37.6 & 972.8 & 1.0 & 235 \\ 510 & 40 & 26.6 & 2 & 37.0 & 973.5 & 1.4 & 105 \\ 532 & 40 & 26.5 & 2 & 37.6 & 974.0 & 0.4 & 341 \\ 630 & 40 & 26.9 & 2 & 37.5 & 974.4 & 0.0 & 46\end{array}$

SITE 122 (August 24, 1970)

(August 18, 1970)

$\begin{array}{rrrrrrrr}102 & 35 & 58.7 & -6 & 55.4 & 382.0 & 10.5 & 101 \\ 202 & 35 & 56.7 & -6 & 42.6 & 392.6 & 11.1 & 97 \\ 348 & 35 & 54.2 & -6 & 18.6 & 412.1 & 10.7 & 87 \\ 436 & 35 & 54.7 & -6 & 08.1 & 420.7 & 11.7 & 95 \\ 521 & 35 & 53.9 & -5 & 57.3 & 429.5 & 12.4 & 86 \\ 541 & 35 & 54.2 & -5 & 52.2 & 433.6 & 12.9 & 90 \\ 601 & 35 & 54.2 & -5 & 46.9 & 437.9 & 13.3 & 89 \\ 627 & 35 & 54.3 & -5 & 39.8 & 443.6 & 13.1 & 76 \\ 655 & 35 & 55.8 & -5 & 32.5 & 449.7 & 12.7 & 73 \\ 729 & 35 & 57.9 & -5 & 24.0 & 456.9 & 13.4 & 76 \\ 800 & 35 & 59.6 & -5 & 15.7 & 463.9 & 13.8 & 85 \\ 914 & 36 & 01.1 & -4 & 54.7 & 480.9 & 12.5 & 79 \\ 1100 & 36 & 05.4 & -4 & 27.9 & 503.0 & 12.6 & 85 \\ 1136 & 36 & 06.0 & -4 & 18.6 & 510.5 & 6.9 & 346 \\ 1228 & 36 & 11.8 & -4 & 20.4 & 516.5 & 6.4 & 347 \\ 1252 & 36 & 14.3 & -4 & 21.1 & 519.1 & 5.9 & 155 \\ 1310 & 36 & 12.7 & -4 & 20.2 & 520.8 & 6.1 & 152 \\ 1320 & 36 & 11.8 & -4 & 19.6 & 521.9 & 2.6 & 252 \\ 1420 & 36 & 11.0 & -4 & 22.7 & 524.5 & 2.9 & 102 \\ 1456 & 36 & 10.6 & -4 & 20.6 & 526.2 & 2.6 & 101 \\ 1520 & 36 & 10.4 & -4 & 19.3 & 527.3 & 3.7 & 254 \\ 1610 & 36 & 09.6 & -4 & 23.0 & 530.3 & 1.4 & 86 \\ 1630 & 36 & 09.6 & -4 & 22.4 & 530.8 & 0.0 & 90\end{array}$

SITE 121 (August 21, 1970)

\begin{tabular}{rrrrrrrr}
1106 & 36 & 09.6 & -4 & 22.4 & 530.8 & 8.2 & 66 \\
1200 & 36 & 12.6 & -4 & 14.0 & 538.2 & 12.0 & 78 \\
1402 & 36 & 17.6 & -3 & 44.4 & 562.6 & 11.2 & 76 \\
1548 & 36 & 22.4 & -3 & 20.6 & 582.4 & 10.5 & 79 \\
1816 & 36 & 27.3 & -2 & 48.9 & 608.3 & 11.3 & 80 \\
2128 & 36 & 33.9 & -2 & 04.7 & 644.5 & 11.4 & 55 \\
2136 & 36 & 34.8 & -2 & 03.2 & 646.0 & 11.8 & 57 \\
2222 & 36 & 39.7 & -1 & 53.8 & 655.0 & 10.7 & 50 \\
2322 & 36 & 46.6 & -1 & 43.6 & 665.7 & 9.7 & 44 \\
\hline
\end{tabular}

a West longitudes are negative, speed in knots, cumulative distance in

$\begin{array}{llll}620 & 40 & 26.9\end{array}$

$\begin{array}{lll}625 & 40 & 27.3\end{array}$

$\begin{array}{lll}715 & 40 & 34.1\end{array}$

$\begin{array}{llll}751 & 40 & 38.5\end{array}$

$\begin{array}{lll}754 & 40 & 38.5\end{array}$

$\begin{array}{lll}800 & 40 & 38.1\end{array}$

$\begin{array}{lll}813 & 40 & 37.8\end{array}$

$\begin{array}{lll}825 & 40 & 37.8\end{array}$

$\begin{array}{lll}840 & 40 & 37.8\end{array}$
974.4

974.9

983.7

989.8

989.9

990.4

991.1

991.3

991.5

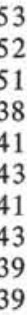

SITE 123 (August 25, 1970)

$\begin{array}{rrrrrrrr}2230 & 40 & 37.8 & 2 & 50.3 & 991.6 & 4.0 & 320 \\ 2249 & 40 & 38.8 & 2 & 49.2 & 992.8 & 4.6 & 30 \\ 2252 & 40 & 39.0 & 2 & 49.3 & 993.0 & 7.9 & 140 \\ 2256 & 40 & 38.6 & 2 & 49.8 & 993.6 & 7.3 & 155 \\ 2303 & 40 & 37.8 & 2 & 50.3 & 994.4 & 11.3 & 132\end{array}$

(August 26, 1970)

\begin{tabular}{rrrrrrrr}
010 & 40 & 29.3 & 3 & 02.6 & 1007.1 & 9.5 & 146 \\
150 & 40 & 16.1 & 3 & 14.2 & 1022.9 & 10.8 & 137 \\
302 & 40 & 06.6 & 3 & 25.8 & 1036.0 & 10.2 & 139 \\
448 & 39 & 53.0 & 3 & 41.1 & 1053.9 & 10.0 & 135 \\
802 & 39 & 30.2 & 4 & 10.7 & 1086.1 & 9.3 & 136 \\
851 & 39 & 24.8 & 4 & 17.6 & 1093.7 & 9.1 & 143 \\
946 & 39 & 18.1 & 4 & 24.1 & 1102.1 & 10.2 & 139 \\
1116 & 39 & 06.6 & 4 & 37.0 & 1117.3 & 8.1 & 142 \\
1132 & 39 & 04.9 & 4 & 38.7 & 1119.5 & 10.9 & 137 \\
1138 & 39 & 04.1 & 4 & 39.7 & 1120.6 & 9.4 & 111 \\
1300 & 38 & 59.6 & 4 & 55.1 & 1133.4 & 10.6 & 109 \\
1310 & 38 & 59.0 & 4 & 57.3 & 1135.2 & 3.9 & 111 \\
1349 & 38 & 58.1 & 5 & 00.3 & 1137.7 & 5.7 & 156 \\
1410 & 38 & 56.3 & 5 & 01.4 & 1139.7 & 6.1 & 159 \\
1424 & 38 & 54.9 & 5 & 02.0 & 1141.1 & 5.2 & 151 \\
1436 & 38 & 54.0 & 5 & 02.7 & 1142.2 & 5.7 & 142 \\
1446 & 38 & 53.3 & 5 & 03.4 & 1143.1 & 5.5 & 143 \\
1505 & 38 & 51.9 & 5 & 04.8 & 1144.9 & 5.3 & 257 \\
1510 & 38 & 51.8 & 5 & 04.2 & 1145.3 & 8.6 & 276 \\
1524 & 38 & 52.0 & 5 & 01.7 & 1147.3 & 8.1 & 284 \\
\hline
\end{tabular}


TABLE 1 - Continued

\begin{tabular}{|c|c|c|c|c|c|}
\hline Time & Latitude & Longitude & $\begin{array}{c}\text { Distance } \\
\text { (miles) }\end{array}$ & $\begin{array}{c}\text { Speed } \\
\text { (knots) }\end{array}$ & $\begin{array}{c}\text { Course } \\
\text { (degrees) }\end{array}$ \\
\hline 1530 & $\begin{array}{ll}38 & 52.2\end{array}$ & $\begin{array}{ll}5 & 00.7\end{array}$ & 1148.1 & 3.8 & 279 \\
\hline 1545 & $\begin{array}{ll}38 & 52.3\end{array}$ & 459.5 & 1149.1 & 0.9 & 237 \\
\hline 1551 & $\begin{array}{ll}38 & 52.3\end{array}$ & $\begin{array}{ll}4 & 59.4\end{array}$ & 1149.2 & 3.0 & 98 \\
\hline 1605 & $\begin{array}{ll}38 & 52.2\end{array}$ & $\begin{array}{ll}5 & 00.3\end{array}$ & 1149.9 & 0.6 & 291 \\
\hline 1700 & $38 \quad 52.4$ & $\begin{array}{ll}4 & 59.7\end{array}$ & 1150.4 & 0.0 & 21 \\
\hline
\end{tabular}

SITE 124 (August 29, 1970)

$\begin{array}{rrrrrrrr}1820 & 38 & 52.4 & 4 & 59.7 & 1150.4 & 4.0 & 90 \\ 1835 & 38 & 52.4 & 5 & 01.0 & 1151.4 & 10.4 & 93 \\ 1926 & 38 & 51.9 & 5 & 12.3 & 1160.2 & 10.4 & 98 \\ 2112 & 38 & 49.5 & 5 & 35.7 & 1178.6 & 10.9 & 100 \\ 2154 & 38 & 48.2 & 5 & 45.3 & 1186.2 & 9.2 & 96 \\ 2218 & 38 & 47.8 & 5 & 50.0 & 1189.9 & 10.5 & 90\end{array}$

(August 30, 1970)

$\begin{array}{rrr}136 & 38 & 47.5 \\ 200 & 38 & 47.5 \\ 508 & 38 & 47.3 \\ 804 & 38 & 46.6 \\ 918 & 38 & 46.3 \\ 1117 & 38 & 45.5 \\ 1620 & 38 & 25.9 \\ 2100 & 38 & 11.5 \\ 2208 & 38 & 07.6\end{array}$

$\begin{array}{rrr}6 & 34.5 & 1224.6 \\ 6 & 41.0 & 1229.6 \\ 7 & 27.0 & 1265.5 \\ 8 & 07.6 & 1297.1 \\ 8 & 24.8 & 1310.6 \\ 8 & 50.1 & 1330.3 \\ 9 & 53.9 & 1383.9 \\ 10 & 54.8 & 1433.8 \\ 11 & 09.8 & 1446.2\end{array}$

$\begin{array}{rr}12.6 & 90 \\ 11.4 & 90 \\ 10.8 & 91 \\ 10.9 & 91 \\ 10.0 & 92 \\ 10.6 & 111 \\ 10.7 & 107 \\ 11.0 & 109 \\ 11.2 & 113\end{array}$

\section{(August 31, 1970)}

$\begin{array}{rrrrrrrr}048 & 37 & 56.1 & 11 & 44.6 & 1476.0 & 11.1 & 123 \\ 230 & 37 & 45.9 & 12 & 04.8 & 1494.9 & 11.0 & 125 \\ 416 & 37 & 34.6 & 12 & 24.8 & 1514.3 & 9.9 & 121 \\ 554 & 37 & 26.4 & 12 & 42.5 & 1530.6 & 9.9 & 122 \\ 730 & 37 & 18.1 & 12 & 59.4 & 1546.4 & 10.3 & 123 \\ 858 & 37 & 09.9 & 13 & 15.3 & 1561.5 & 10.4 & 123 \\ 1156 & 36 & 53.2 & 13 & 47.9 & 1592.4 & 10.1 & 116 \\ 1217 & 36 & 51.7 & 13 & 51.9 & 1595.9 & 12.8 & 116 \\ 1342 & 36 & 43.7 & 14 & 12.2 & 1614.0 & 11.5 & 117 \\ 1530 & 36 & 34.2 & 14 & 35.0 & 1634.7 & 10.9 & 115 \\ 1715 & 36 & 26.0 & 14 & 56.5 & 1653.8 & 10.7 & 115 \\ 2118 & 36 & 07.3 & 15 & 45.2 & 1697.2 & 10.0 & 115 \\ 2352 & 35 & 56.4 & 16 & 14.0 & 1723.0 & 9.7 & 113\end{array}$

(September 1, 1970)

\begin{tabular}{rrrrrrrr}
138 & 35 & 49.8 & 16 & 33.5 & 1740.1 & 11.9 & 129 \\
146 & 35 & 48.8 & 16 & 35.0 & 1741.6 & 10.8 & 112 \\
326 & 35 & 41.9 & 16 & 55.6 & 1759.7 & 10.6 & 111 \\
806 & 35 & 24.4 & 17 & 52.4 & 1809.1 & 11.3 & 110 \\
956 & 35 & 17.4 & 18 & 16.2 & 1829.8 & 11.1 & 115 \\
1102 & 35 & 12.2 & 18 & 29.8 & 1842.1 & 11.4 & 115 \\
1250 & 35 & 03.6 & 18 & 52.5 & 1862.5 & 11.3 & 112 \\
1442 & 34 & 55.8 & 19 & 16.4 & 1883.6 & 11.0 & 113 \\
1525 & 34 & 52.7 & 19 & 25.3 & 1891.5 & 10.1 & 105 \\
1700 & 34 & 48.7 & 19 & 44.1 & 1907.5 & 10.4 & 105 \\
1848 & 34 & 43.9 & 20 & 06.2 & 1926.2 & 11.8 & 102 \\
1920 & 34 & 42.6 & 20 & 13.7 & 1932.5 & 10.8 & 103 \\
1959 & 34 & 41.1 & 20 & 22.0 & 1939.5 & 10.9 & 137 \\
2012 & 34 & 39.4 & 20 & 23.9 & 1941.9 & 4.1 & 137 \\
2028 & 34 & 38.6 & 20 & 24.9 & 1943.0 & 6.8 & 137 \\
2040 & 34 & 37.6 & 20 & 26.0 & 1944.3 & 2.6 & 220 \\
2043 & 34 & 37.5 & 20 & 25.9 & 1944.4 & 2.5 & 307 \\
2053 & 34 & 37.7 & 20 & 25.5 & 1944.9 & 1.6 & 141 \\
2058 & 34 & 37.6 & 20 & 25.6 & 1945.0 & 1.0 & 141 \\
2102 & 34 & 37.6 & 20 & 25.6 & 1945.1 & 0.1 & 140 \\
2200 & 34 & 37.5 & 20 & 25.8 & 1945.2 & 0.0 & 321 \\
& & & & & & & \\
SITE & 125 & (September 4, 1970 ) & & & \\
445 & 34 & 37.5 & 20 & 25.7 & 1945.2 & 6.2 & 84 \\
455 & 34 & 37.6 & 20 & 27.0 & 1946.2 & 10.6 & 89 \\
\hline
\end{tabular}

TABLE 1 - Continued

\begin{tabular}{|c|c|c|c|c|c|}
\hline Time & Latitude & Longitude & $\begin{array}{l}\text { Distance } \\
\text { (miles }\end{array}$ & $\begin{array}{c}\text { Speed } \\
\text { (knots) }\end{array}$ & $\begin{array}{c}\text { Course } \\
\text { (degrees) }\end{array}$ \\
\hline 550 & $34 \quad 37.8$ & $20 \quad 38.8$ & 1956.0 & 10.8 & 56 \\
\hline 602 & $34 \quad 39.0$ & $20 \quad 41.0$ & 1958.1 & 9.7 & 50 \\
\hline 718 & $34 \quad 46.9$ & $20 \quad 52.4$ & 1970.4 & 10.1 & 47 \\
\hline 750 & $34 \quad 50.6$ & $\begin{array}{ll}20 & 57.2\end{array}$ & 1975.8 & 10.6 & 48 \\
\hline 910 & $34 \quad 60.0$ & $21 \quad 10.2$ & 1990.0 & 10.2 & 51 \\
\hline 949 & $\begin{array}{ll}35 & 04.1\end{array}$ & $21 \quad 16.5$ & 1996.6 & 10.4 & 55 \\
\hline 953 & $35 \quad 04.5$ & $21 \quad 17.2$ & 1997.3 & 10.3 & 53 \\
\hline 1043 & $\begin{array}{ll}35 & 09.7\end{array}$ & 2125.6 & 2005.9 & 5.0 & 56 \\
\hline 1057 & $\begin{array}{ll}35 & 10.3\end{array}$ & $21 \quad 26.8$ & 2007.1 & 1.9 & 121 \\
\hline 1100 & $\begin{array}{ll}35 & 10.3\end{array}$ & $\begin{array}{ll}21 & 26.9\end{array}$ & 2007.2 & 4.9 & 235 \\
\hline 1117 & $\begin{array}{ll}35 & 09.5\end{array}$ & $21 \quad 25.5$ & 2008.6 & 1.2 & 22 \\
\hline 1128 & $\begin{array}{ll}35 & 09.7\end{array}$ & 2125.6 & 2008.8 & 0.0 & 39 \\
\hline 1200 & $\begin{array}{ll}35 & 09.7\end{array}$ & 2125.6 & 2008.8 & 0.0 & 219 \\
\hline
\end{tabular}

SITE 126 (September 6, 1972)

$\begin{array}{rrrrrrrr}445 & 35 & 09.5 & 21 & 25.4 & 2009.1 & 4.2 & 90 \\ 452 & 35 & 09.5 & 21 & 26.0 & 2009.6 & 9.7 & 87 \\ 553 & 35 & 10.0 & 21 & 38.0 & 2019.4 & 10.6 & 55 \\ 612 & 35 & 11.9 & 21 & 41.4 & 2022.8 & 9.9 & 49 \\ 724 & 35 & 19.8 & 21 & 52.4 & 2034.7 & 9.6 & 51 \\ 758 & 35 & 23.2 & 21 & 57.6 & 2040.2 & 10.4 & 48 \\ 836 & 35 & 27.6 & 22 & 03.6 & 2046.7 & 10.1 & 51 \\ 910 & 35 & 31.2 & 22 & 09.1 & 2052.5 & 10.1 & 54 \\ 1002 & 35 & 36.3 & 22 & 17.8 & 2061.2 & 10.3 & 54 \\ 1050 & 35 & 41.1 & 22 & 26.0 & 2069.4 & 9.3 & 48 \\ 1117 & 35 & 43.9 & 22 & 29.8 & 2073.6 & 5.2 & 50 \\ 1133 & 35 & 44.8 & 22 & 31.1 & 2075.0 & 6.9 & 152 \\ 1136 & 35 & 44.5 & 22 & 31.3 & 2075.3 & 8.0 & 244 \\ 1146 & 35 & 43.9 & 22 & 29.8 & 2076.6 & 0.0 & 240 \\ 1209 & 35 & 43.9 & 22 & 29.8 & 2076.7 & 0.0 & 227\end{array}$

SITES 127 AND 128 (September 12, 1970)

$\begin{array}{llllllrl}1327 & 35 & 42.6 & 22 & 28.1 & 2078.6 & 5.9 & 121 \\ 1353 & 35 & 41.3 & 22 & 30.8 & 2081.1 & 11.0 & 121 \\ 1411 & 35 & 39.6 & 22 & 34.3 & 2084.4 & 7.0 & 119 \\ 1440 & 35 & 38.0 & 22 & 38.0 & 2087.8 & 6.2 & 127 \\ 1500 & 35 & 36.7 & 22 & 40.0 & 2089.9 & 8.6 & 122 \\ 1710 & 35 & 26.7 & 22 & 59.4 & 2108.6 & 10.6 & 126 \\ 1848 & 35 & 16.6 & 23 & 16.7 & 2125.9 & 9.6 & 115 \\ 2018 & 35 & 10.5 & 23 & 32.6 & 2140.3 & 9.6 & 110 \\ 2152 & 35 & 05.4 & 23 & 49.8 & 2155.3 & 10.8 & 110 \\ 2215 & 35 & 04.0 & 23 & 54.6 & 2159.4 & 10.1 & 108 \\ 2334 & 35 & 00.0 & 24 & 10.0 & 2172.7 & 10.4 & 105\end{array}$

(September 13, 1970)

\begin{tabular}{rrrrrrrr}
052 & 34 & 56.4 & 24 & 26.0 & 2186.3 & 11.2 & 109 \\
105 & 34 & 55.6 & 24 & 28.8 & 2188.7 & 11.4 & 112 \\
119 & 34 & 54.6 & 24 & 31.8 & 2191.3 & 10.2 & 117 \\
240 & 34 & 48.4 & 24 & 46.8 & 2205.1 & 10.1 & 117 \\
314 & 34 & 45.8 & 24 & 53.0 & 2210.8 & 10.1 & 103 \\
550 & 34 & 39.9 & 25 & 24.0 & 2237.0 & 9.8 & 101 \\
644 & 34 & 38.2 & 25 & 34.6 & 2245.8 & 11.2 & 91 \\
738 & 34 & 38.1 & 25 & 46.8 & 2255.9 & 10.1 & 90 \\
832 & 34 & 38.1 & 25 & 57.9 & 2265.0 & 10.3 & 87 \\
858 & 34 & 38.4 & 26 & 03.3 & 2269.5 & 11.0 & 92 \\
1044 & 34 & 37.8 & 26 & 27.0 & 2289.0 & 11.2 & 88 \\
1115 & 34 & 38.0 & 26 & 34.0 & 2294.8 & 11.8 & 123 \\
1204 & 34 & 32.7 & 26 & 43.8 & 2304.4 & 11.7 & 124 \\
1350 & 34 & 21.1 & 27 & 04.6 & 2325.1 & 11.1 & 131 \\
1352 & 34 & 20.9 & 27 & 04.9 & 2325.4 & 8.8 & 145 \\
1355 & 34 & 20.5 & 27 & 05.2 & 2325.9 & 6.7 & 144 \\
1403 & 34 & 19.8 & 27 & 05.8 & 2326.8 & 4.7 & 190 \\
1406 & 34 & 19.6 & 27 & 05.8 & 2327.0 & 5.6 & 278 \\
1415 & 34 & 19.7 & 27 & 04.8 & 2327.8 & 2.4 & 21 \\
1419 & 34 & 19.8 & 27 & 04.9 & 2328.0 & 5.3 & 44 \\
1437 & 34 & 21.0 & 27 & 06.2 & 2329.6 & 4.3 & 357 \\
1447 & 34 & 21.7 & 27 & 06.2 & 2330.3 & 1.6 & 348 \\
1502 & 34 & 22.1 & 27 & 06.1 & 2330.7 & 3.0 & 300 \\
\hline
\end{tabular}


TABLE 1 - Continued

\begin{tabular}{|c|c|c|c|c|c|}
\hline Time & Latitude & Longitude & $\begin{array}{c}\text { Distance } \\
\text { (miles) }\end{array}$ & $\begin{array}{l}\text { Speed } \\
\text { (knots) }\end{array}$ & $\begin{array}{c}\text { Course } \\
\text { (degrees) }\end{array}$ \\
\hline 1506 & $34 \quad 22.2$ & $27 \quad 05.9$ & 2330.9 & 3.3 & 216 \\
\hline 1510 & $\begin{array}{ll}34 & 22.0\end{array}$ & $\begin{array}{ll}27 & 05.7\end{array}$ & 2331.2 & 3.9 & 187 \\
\hline 1520 & $\begin{array}{ll}34 & 21.4\end{array}$ & $27 \quad 05.6$ & 2331.8 & 2.9 & 205 \\
\hline 1528 & 3421.0 & $27 \quad 05.4$ & 2332.2 & 3.1 & 278 \\
\hline 1532 & $34 \quad 21.1$ & 2705.1 & 2332.4 & 1.0 & 260 \\
\hline 1543 & $34 \quad 21.0$ & $27 \quad 04.9$ & 2332.6 & 0.3 & 190 \\
\hline 1600 & 3421.0 & $27 \quad 04.9$ & 2332.7 & 0.0 & 245 \\
\hline
\end{tabular}

SITE 129 (September 16, 1970)

$\begin{array}{rrrrrrrr}345 & 34 & 20.8 & 27 & 04.5 & 2333.0 & 5.4 & 153 \\ 355 & 34 & 20.0 & 27 & 05.0 & 2333.9 & 11.2 & 131 \\ 556 & 34 & 05.1 & 27 & 25.4 & 2356.5 & 11.0 & 132 \\ 633 & 34 & 00.6 & 27 & 31.5 & 2363.2 & 10.8 & 99 \\ 640 & 34 & 00.4 & 27 & 33.0 & 2364.5 & 10.9 & 137 \\ 717 & 33 & 55.5 & 27 & 38.6 & 2371.2 & 11.0 & 136 \\ 742 & 33 & 52.2 & 27 & 42.4 & 2375.8 & 11.2 & 140 \\ 821 & 33 & 46.6 & 27 & 48.1 & 2383.1 & 11.8 & 166 \\ 912 & 33 & 36.9 & 27 & 51.1 & 2393.1 & 6.2 & 167 \\ 926 & 33 & 35.5 & 27 & 51.5 & 2394.6 & 4.1 & 68 \\ 930 & 33 & 35.6 & 27 & 51.8 & 2394.9 & 2.8 & 0 \\ 945 & 33 & 36.3 & 27 & 51.8 & 2395.6 & 0.6 & 86 \\ 1000 & 33 & 36.3 & 27 & 52.0 & 2395.7 & 0.0 & 90\end{array}$

SITE 130 (September 18, 1970)

$\begin{array}{rrrrrrrr}710 & 33 & 36.3 & 27 & 52.0 & 2395.7 & 4.2 & 152 \\ 728 & 33 & 35.2 & 27 & 52.7 & 2397.0 & 10.7 & 131 \\ 748 & 33 & 32.8 & 27 & 55.9 & 2400.6 & 11.0 & 131 \\ 946 & 33 & 18.8 & 28 & 15.6 & 2422.2 & 11.2 & 132 \\ 1016 & 33 & 15.0 & 28 & 20.6 & 2427.8 & 10.5 & 138 \\ 1128 & 33 & 05.6 & 28 & 30.6 & 2440.3 & 10.7 & 142 \\ 1129 & 33 & 05.5 & 28 & 30.7 & 2440.5 & 4.2 & 140 \\ 1142 & 33 & 04.8 & 28 & 31.4 & 2441.4 & 4.8 & 141 \\ 1147 & 33 & 04.5 & 28 & 31.7 & 2441.8 & 3.1 & 40 \\ 1152 & 33 & 04.7 & 28 & 31.9 & 2442.1 & 5.7 & 328 \\ 1210 & 33 & 06.1 & 28 & 30.8 & 2443.8 & 0.8 & 339 \\ 1230 & 33 & 06.4 & 28 & 30.7 & 2444.1 & 0.0 & 40\end{array}$

SITE 131 (September 20, 1970)

\begin{tabular}{rrlrrrrr}
001 & 33 & 06.4 & 28 & 30.7 & 2444.1 & 5.8 & 294 \\
032 & 33 & 07.6 & 28 & 27.4 & 2447.1 & 10.0 & 293 \\
344 & 33 & 20.0 & 27 & 52.2 & 2479.0 & 8.4 & 282 \\
424 & 33 & 21.1 & 27 & 45.6 & 2484.6 & 8.9 & 291 \\
440 & 33 & 22.0 & 27 & 43.0 & 2487.0 & 10.8 & 285 \\
526 & 33 & 24.1 & 27 & 33.4 & 2495.3 & 8.8 & 290 \\
604 & 33 & 26.0 & 27 & 27.2 & 2500.8 & 9.2 & 289 \\
714 & 33 & 29.5 & 27 & 15.0 & 2511.6 & 10.0 & 281 \\
738 & 33 & 30.3 & 27 & 10.3 & 2515.5 & 9.8 & 301 \\
752 & 33 & 31.5 & 27 & 07.9 & 2517.8 & 9.4 & 282 \\
838 & 33 & 33.0 & 26 & 59.5 & 2525.0 & 9.5 & 297 \\
938 & 33 & 37.3 & 26 & 49.3 & 2534.6 & 8.6 & 291 \\
944 & 33 & 37.6 & 26 & 48.3 & 2535.4 & 4.7 & 301 \\
949 & 33 & 37.8 & 26 & 47.9 & 2535.8 & 8.5 & 296 \\
1126 & 33 & 43.9 & 26 & 33.0 & 2549.6 & 9.5 & 297 \\
1146 & 33 & 45.3 & 26 & 29.6 & 2552.8 & 9.7 & 294 \\
1334 & 33 & 52.4 & 26 & 10.3 & 2570.3 & 9.7 & 294 \\
1400 & 33 & 54.1 & 26 & 05.7 & 2574.5 & 10.9 & 286 \\
1718 & 34 & 04.3 & 25 & 24.0 & 2610.6 & 10.4 & 286 \\
1804 & 34 & 06.5 & 25 & 14.8 & 2618.5 & 10.2 & 292 \\
1908 & 34 & 10.7 & 25 & 02.6 & 2629.4 & 10.8 & 289 \\
2134 & 34 & 19.2 & 24 & 32.4 & 2655.8 & 11.4 & 290 \\
2320 & 34 & 26.1 & 24 & 09.5 & 2675.9 & 12.1 & 291 \\
2348 & 34 & 28.1 & 24 & 03.1 & 2681.5 & 9.0 & 288 \\
& & & & & & & \\
& (September 21,1970$)$ & & & \\
001 & 34 & 28.7 & 24 & 00.9 & 2683.5 & 11.3 & 299 \\
128 & 34 & 36.8 & 23 & 43.5 & 2699.9 & 10.6 & 302 \\
\hline
\end{tabular}

TABLE 1 - Continued

\begin{tabular}{|c|c|c|c|c|c|}
\hline Time & Latitude & Longitude & $\begin{array}{c}\text { Distance } \\
\text { (miles) }\end{array}$ & $\begin{array}{c}\text { Speed } \\
\text { (knots) }\end{array}$ & $\begin{array}{c}\text { Course } \\
\text { (degrees) }\end{array}$ \\
\hline 215 & $34 \quad 41.2$ & $23 \quad 35.0$ & 2708.2 & 6.1 & 295 \\
\hline 414 & $34 \quad 46.4$ & 2321.6 & 2720.4 & 10.0 & 282 \\
\hline 438 & $\begin{array}{ll}34 & 47.2\end{array}$ & $23 \quad 16.8$ & 2724.4 & 9.6 & 290 \\
\hline 516 & $34 \quad 49.3$ & $23 \quad 09.9$ & 2730.5 & 9.3 & 288 \\
\hline 624 & $34 \quad 52.6$ & $22 \quad 57.6$ & 2741.0 & 11.4 & 284 \\
\hline 700 & $34 \quad 54.2$ & $22 \quad 49.5$ & 2747.8 & 9.3 & 283 \\
\hline 730 & $34 \quad 55.2$ & $22 \quad 44.0$ & 2752.5 & 10.5 & 291 \\
\hline 844 & $34 \quad 59.8$ & $22 \quad 29.2$ & 2765.5 & 10.5 & 288 \\
\hline 1024 & $\begin{array}{ll}35 & 05.1\end{array}$ & $22 \quad 08.9$ & 2782.9 & 9.2 & 300 \\
\hline 1058 & $\begin{array}{ll}35 & 07.7\end{array}$ & $22 \quad 03.3$ & 2788.2 & 10.1 & 297 \\
\hline 1143 & $35 \quad 11.1$ & 2155.1 & 2795.7 & 9.6 & 300 \\
\hline 1242 & $\begin{array}{ll}35 & 15.9\end{array}$ & $21 \quad 45.1$ & 2805.2 & 9.7 & 292 \\
\hline 1430 & $35 \quad 22.5$ & $21 \quad 25.4$ & 2822.6 & 9.9 & 291 \\
\hline 1510 & $\begin{array}{ll}35 & 24.9\end{array}$ & 2117.8 & 2829.2 & 8.9 & 288 \\
\hline 1816 & $\begin{array}{ll}35 & 33.5\end{array}$ & $20 \quad 45.5$ & 2856.9 & 10.2 & 283 \\
\hline 2014 & $\begin{array}{ll}35 & 37.9\end{array}$ & $20 \quad 21.4$ & 2877.0 & 8.7 & 293 \\
\hline 2258 & $\begin{array}{ll}35 & 47.3\end{array}$ & 1954.6 & 2900.7 & 9.1 & 290 \\
\hline
\end{tabular}

(September 22, 1970)

$\begin{array}{rrrrrrrr}042 & 35 & 52.8 & 19 & 36.4 & 2916.4 & 9.0 & 290 \\ 125 & 35 & 55.0 & 19 & 28.9 & 2922.9 & 10.0 & 296 \\ 224 & 35 & 59.3 & 19 & 18.0 & 2932.7 & 9.4 & 293 \\ 303 & 36 & 01.7 & 19 & 11.1 & 2938.8 & 9.1 & 297 \\ 418 & 36 & 06.9 & 18 & 58.5 & 2950.2 & 9.3 & 287 \\ 438 & 36 & 07.8 & 18 & 54.9 & 2953.2 & 8.7 & 283 \\ 538 & 36 & 09.8 & 18 & 44.5 & 2961.9 & 6.9 & 288 \\ 602 & 36 & 10.6 & 18 & 41.2 & 2964.7 & 9.5 & 288 \\ 610 & 36 & 11.0 & 18 & 39.7 & 2965.9 & 9.8 & 286 \\ 700 & 36 & 13.3 & 18 & 30.0 & 2974.1 & 9.3 & 292 \\ 722 & 36 & 14.6 & 18 & 26.1 & 2977.5 & 9.0 & 299 \\ 750 & 36 & 16.6 & 18 & 21.5 & 2981.7 & 7.1 & 308 \\ 950 & 36 & 25.4 & 18 & 07.7 & 2995.9 & 4.1 & 314 \\ 1021 & 36 & 26.9 & 18 & 05.8 & 2998.0 & 0.2 & 305 \\ 1120 & 36 & 27.0 & 18 & 05.5 & 2998.3 & 2.2 & 290 \\ 1156 & 36 & 27.5 & 18 & 04.0 & 2999.6 & 1.5 & 5 \\ 1310 & 36 & 29.3 & 18 & 04.2 & 3001.4 & 2.7 & 346 \\ 1333 & 36 & 30.3 & 18 & 03.9 & 3002.5 & 7.5 & 300 \\ 1340 & 36 & 30.7 & 18 & 03.0 & 3003.3 & 7.2 & 308 \\ 1608 & 36 & 41.7 & 17 & 45.7 & 3021.0 & 8.4 & 305 \\ 1926 & 36 & 57.5 & 17 & 17.2 & 3048.7 & 9.4 & 305 \\ 2130 & 37 & 08.5 & 16 & 57.3 & 3068.1 & 9.4 & 306 \\ 2318 & 37 & 18.5 & 16 & 40.2 & 3085.0 & 9.4 & 308 \\ 2354 & 37 & 21.9 & 16 & 34.6 & 3090.6 & 9.8 & 303\end{array}$

(September 23, 1970)

\begin{tabular}{rrrrrrrr}
030 & 37 & 25.1 & 16 & 28.4 & 3096.4 & 9.5 & 312 \\
140 & 37 & 32.4 & 16 & 18.0 & 3107.5 & 9.7 & 309 \\
209 & 37 & 35.4 & 16 & 13.4 & 3112.2 & 11.1 & 298 \\
244 & 37 & 38.4 & 16 & 06.2 & 3118.6 & 10.0 & 292 \\
322 & 37 & 40.7 & 15 & 58.8 & 3124.9 & 11.1 & 292 \\
346 & 37 & 42.4 & 15 & 53.6 & 3129.4 & 9.4 & 302 \\
415 & 37 & 44.8 & 15 & 48.7 & 3133.9 & 9.5 & 299 \\
436 & 37 & 46.4 & 15 & 45.0 & 3137.3 & 10.9 & 300 \\
447 & 37 & 47.4 & 15 & 42.8 & 3139.3 & 10.4 & 319 \\
507 & 37 & 50.0 & 15 & 39.9 & 3142.7 & 10.5 & 329 \\
537 & 37 & 54.5 & 15 & 36.5 & 3148.0 & 9.2 & 349 \\
610 & 37 & 59.5 & 15 & 35.3 & 3153.1 & 10.4 & 2 \\
621 & 38 & 01.4 & 15 & 35.4 & 3155.0 & 11.7 & 2 \\
640 & 38 & 05.1 & 15 & 35.6 & 3158.7 & 5.4 & 5 \\
700 & 38 & 06.9 & 15 & 35.8 & 3160.5 & 6.4 & 4 \\
735 & 38 & 10.6 & 15 & 36.1 & 3164.2 & 5.5 & 12 \\
805 & 38 & 13.3 & 15 & 36.8 & 3166.9 & 6.9 & 38 \\
815 & 38 & 14.2 & 15 & 37.7 & 3168.1 & 10.5 & 83 \\
824 & 38 & 14.4 & 15 & 39.7 & 3169.7 & 9.7 & 47 \\
834 & 38 & 15.5 & 15 & 41.2 & 3171.3 & 6.4 & 21 \\
842 & 38 & 16.3 & 15 & 41.6 & 3172.1 & 9.1 & 351 \\
852 & 38 & 17.8 & 15 & 41.3 & 3173.6 & 10.0 & 318 \\
942 & 38 & 24.0 & 15 & 34.2 & 3182.0 & 9.3 & 306 \\
\hline
\end{tabular}


TABLE 1 - Continued

\begin{tabular}{|c|c|c|c|c|c|c|c|}
\hline \multirow{2}{*}{$\frac{\text { Time }}{1026}$} & \multicolumn{2}{|c|}{ Latitude } & \multicolumn{2}{|c|}{ Longitude } & \multirow{2}{*}{$\begin{array}{c}\begin{array}{c}\text { Distance } \\
\text { (miles) }\end{array} \\
3188.8\end{array}$} & \multirow{2}{*}{$\begin{array}{c}\begin{array}{c}\text { Speed } \\
\text { (knots) }\end{array} \\
9.5\end{array}$} & \multirow{2}{*}{$\begin{array}{c}\begin{array}{c}\text { Course } \\
\text { (degrees) }\end{array} \\
319\end{array}$} \\
\hline & 38 & 28.0 & 15 & 27.2 & & & \\
\hline 1250 & 38 & 45.3 & 15 & 08.3 & 3211.5 & 10.1 & 296 \\
\hline 1442 & 38 & 53.5 & 14 & 46.6 & 3230.3 & 10.6 & 298 \\
\hline 1528 & 38 & 57.4 & 14 & 37.4 & 3238.5 & 10.0 & 299 \\
\hline 1648 & 39 & 03.9 & 14 & 22.4 & 3251.8 & 12.4 & 293 \\
\hline 1712 & 39 & 05.8 & 14 & 16.5 & 3256.7 & 10.2 & 296 \\
\hline 1818 & 39 & 10.7 & 14 & 03.5 & 3268.0 & 10.3 & 299 \\
\hline 2008 & 39 & 20.0 & 13 & 42.3 & 3286.8 & 11.3 & 302 \\
\hline 2042 & 39 & 23.4 & 13 & 35.3 & 3293.2 & 10.3 & 301 \\
\hline 2226 & 39 & 32.5 & 13 & 15.4 & 3311.0 & 10.5 & 304 \\
\hline 2248 & 39 & 34.6 & 13 & 11.3 & 3314.9 & 10.5 & 297 \\
\hline
\end{tabular}

(September 24, 1970)

$\begin{array}{rrrrrrrr}010 & 39 & 41.1 & 12 & 54.7 & 3329.2 & 10.4 & 298 \\ 052 & 39 & 44.5 & 12 & 46.3 & 3336.5 & 9.1 & 296 \\ 225 & 39 & 50.8 & 12 & 29.9 & 3350.6 & 4.7 & 303 \\ 232 & 39 & 51.1 & 12 & 29.3 & 3351.1 & 9.2 & 303 \\ 244 & 39 & 52.1 & 12 & 27.3 & 3353.0 & 8.8 & 296 \\ 410 & 39 & 57.6 & 12 & 12.5 & 3365.6 & 9.7 & 299 \\ 544 & 40 & 05.0 & 11 & 55.1 & 3380.8 & 9.0 & 300 \\ 616 & 40 & 07.4 & 11 & 49.7 & 3385.6 & 9.6 & 296 \\ 730 & 40 & 12.7 & 11 & 35.8 & 3397.5 & 10.4 & 293 \\ 800 & 40 & 14.7 & 11 & 29.5 & 3402.7 & 10.3 & 293 \\ 816 & 40 & 15.8 & 11 & 26.2 & 3405.4 & 5.3 & 294 \\ 837 & 40 & 16.5 & 11 & 23.9 & 3407.3 & 2.8 & 14 \\ 841 & 40 & 16.7 & 11 & 24.0 & 3407.5 & 8.8 & 119 \\ 855 & 40 & 15.7 & 11 & 26.3 & 3409.5 & 0.2 & 102 \\ 930 & 40 & 15.7 & 11 & 26.5 & 3409.6 & 0.0 & 90\end{array}$

SITE 132 (September 26, 1970)

$\begin{array}{rrrrrrrr}1015 & 40 & 15.7 & 11 & 26.5 & 3409.6 & 6.0 & 230 \\ 1040 & 40 & 14.1 & 11 & 24.0 & 3412.1 & 10.0 & 227 \\ 1112 & 40 & 10.5 & 11 & 18.9 & 3417.4 & 10.3 & 231 \\ 1149 & 40 & 06.5 & 11 & 12.4 & 3423.8 & 4.8 & 224 \\ 1208 & 40 & 05.4 & 11 & 11.0 & 3425.4 & 10.7 & 230 \\ 1400 & 39 & 52.5 & 10 & 51.2 & 3445.3 & 10.9 & 230 \\ 1730 & 39 & 27.9 & 10 & 13.0 & 3483.6 & 10.8 & 225 \\ 1754 & 39 & 24.9 & 10 & 09.1 & 3487.9 & 12.4 & 231 \\ 1825 & 39 & 20.9 & 10 & 02.6 & 3494.3 & 11.6 & 231 \\ 1916 & 39 & 14.7 & 9 & 52.6 & 3504.2 & 10.3 & 221 \\ 2053 & 39 & 02.2 & 9 & 38.5 & 3520.8 & 10.9 & 247 \\ 2128 & 38 & 59.8 & 9 & 31.0 & 3527.1 & 10.9 & 246 \\ 2312 & 38 & 52.0 & 9 & 08.8 & 3546.1 & 11.4 & 252\end{array}$

(September 27, 1970)

\begin{tabular}{|c|c|c|c|c|c|c|c|}
\hline 014 & 38 & 48.4 & 8 & 54.4 & 3557.8 & 10.8 & 241 \\
\hline 021 & 38 & 47.8 & 8 & 53.0 & 3559.1 & 11.2 & 264 \\
\hline 037 & 38 & 47.5 & 8 & 49.2 & 3562.1 & 11.3 & 267 \\
\hline 225 & 38 & 46.6 & 8 & 23.1 & 3582.4 & 10.6 & 300 \\
\hline 315 & 38 & 51.0 & 8 & 13.3 & 3591.2 & 11.4 & 298 \\
\hline 400 & 38 & 55.0 & 8 & 03.6 & 3599.8 & 10.8 & 299 \\
\hline 504 & 39 & 00.5 & 7 & 50.6 & 3611.3 & 10.4 & 297 \\
\hline 650 & 39 & 08.9 & 7 & 29.5 & 3629.7 & 10.4 & 297 \\
\hline 734 & 39 & 12.4 & 7 & 20.7 & 3637.4 & 5.2 & 297 \\
\hline 802 & 39 & 13.5 & 7 & 17.9 & 3639.8 & 3.7 & 216 \\
\hline 806 & 39 & 13.3 & 7 & 17.7 & 3640.0 & 7.1 & 121 \\
\hline 822 & 39 & 12.3 & 7 & 19.8 & 3641.9 & 5.4 & 121 \\
\hline 827 & 39 & 12.1 & 7 & 20.3 & 3642.4 & 0.3 & 230 \\
\hline 900 & 39 & 12.0 & 7 & 20.1 & 3642.6 & 0.0 & 257 \\
\hline \multicolumn{8}{|c|}{ SITES 133 AND 134 (October 1, 1970) } \\
\hline 1800 & 39 & 11.6 & 7 & 17.9 & 3644.3 & 2.5 & 146 \\
\hline 2000 & 39 & 07.5 & 7 & 21.5 & 3649.3 & 4.6 & 153 \\
\hline 2040 & 39 & 04.8 & 7 & 23.3 & 3652.3 & 8.7 & 257 \\
\hline 2212 & 39 & 01.8 & 7 & 06.6 & 3665.7 & 8.4 & 257 \\
\hline 2348 & 38 & 58.7 & 6 & 49.7 & 3679.1 & 8.8 & 257 \\
\hline
\end{tabular}

TABLE 1 - Continued

\begin{tabular}{|c|c|c|c|c|c|c|c|}
\hline Time & \multicolumn{2}{|c|}{ Latitude } & \multicolumn{2}{|c|}{ Longitude } & $\begin{array}{c}\text { Distance } \\
\text { (miles) }\end{array}$ & $\begin{array}{l}\text { Speed } \\
\text { (knots) }\end{array}$ & $\begin{array}{c}\text { Course } \\
\text { (degrees) }\end{array}$ \\
\hline \multicolumn{8}{|c|}{ (October 2,1970$)$} \\
\hline 114 & 38 & 55.8 & 6 & 34.0 & 3691.7 & 10.0 & 176 \\
\hline 120 & 38 & 54.8 & 6 & 34.1 & 3692.7 & 7.4 & 246 \\
\hline 130 & 38 & 54.3 & 6 & 32.6 & 3693.9 & 6.5 & 247 \\
\hline 307 & 38 & 50.2 & 6 & 20.2 & 3704.4 & 3.2 & 171 \\
\hline 316 & 38 & 49.7 & 6 & 20.3 & 3704.9 & 5.7 & 175 \\
\hline 347 & 38 & 46.8 & 6 & 20.6 & 3707.9 & 8.1 & 256 \\
\hline 350 & 38 & 46.7 & 6 & 20.1 & 3708.3 & 10.1 & 146 \\
\hline 355 & 38 & 46.0 & 6 & 20.7 & 3709.1 & 9.1 & 255 \\
\hline 434 & 38 & 44.4 & 6 & 13.4 & 3715.0 & 6.7 & 256 \\
\hline 632 & 38 & 41.1 & 5 & 57.1 & 3728.2 & 10.1 & 169 \\
\hline 645 & 38 & 39.0 & 5 & 57.6 & 3730.4 & 8.6 & 263 \\
\hline 822 & 38 & 37.3 & 5 & 39.9 & 3744.3 & 9.4 & 264 \\
\hline 918 & 38 & 36.4 & 5 & 28.7 & 3753.1 & 8.9 & 264 \\
\hline 1106 & 38 & 34.6 & 5 & 08.3 & 3769.1 & 10.0 & 257 \\
\hline 1117 & 38 & 34.2 & 5 & 06.0 & 3771.0 & 9.2 & 250 \\
\hline 1244 & 38 & 29.6 & 4 & 49.9 & 3784.4 & 9.2 & 250 \\
\hline 1434 & 38 & 23.8 & 4 & 29.7 & 3801.2 & 9.2 & 253 \\
\hline 1702 & 38 & 17.3 & 4 & 02.2 & 3823.8 & 10.5 & 257 \\
\hline 1746 & 38 & 15.5 & 3 & 52.7 & 3831.5 & 10.0 & 244 \\
\hline 1822 & 38 & 12.9 & 3 & 45.8 & 3837.5 & 9.9 & 252 \\
\hline 1855 & 38 & 11.2 & 3 & 39.2 & 3842.9 & 9.7 & 241 \\
\hline 1928 & 38 & 08.6 & 3 & 33.2 & 3848.3 & 10.8 & 248 \\
\hline 2006 & 38 & 06.1 & 3 & 25.2 & 3855.1 & 10.3 & 246 \\
\hline 2118 & 38 & 01.2 & 3 & 10.9 & 3867.4 & 10.3 & 248 \\
\hline
\end{tabular}

(October 3, 1970)

$\begin{array}{rrrrrrrr}042 & 37 & 47.8 & 2 & 29.9 & 3902.4 & 10.6 & 247 \\ 116 & 37 & 45.5 & 2 & 22.9 & 3908.4 & 10.5 & 242 \\ 234 & 37 & 39.0 & 2 & 07.8 & 3922.0 & 10.2 & 247 \\ 305 & 37 & 37.0 & 2 & 01.6 & 3927.3 & 9.9 & 255 \\ 449 & 37 & 32.5 & 1 & 40.8 & 3944.3 & 9.4 & 247 \\ 530 & 37 & 30.0 & 1 & 33.3 & 3950.8 & 10.3 & 252 \\ 608 & 37 & 28.0 & 1 & 25.5 & 3957.3 & 11.1 & 254 \\ 718 & 37 & 24.4 & 1 & 9.9 & 3970.2 & 10.2 & 251 \\ 911 & 37 & 18.0 & 0 & 47.0 & 3989.5 & 11.5 & 259 \\ 922 & 37 & 17.6 & 0 & 44.4 & 3991.6 & 11.1 & 249 \\ 940 & 37 & 16.4 & 0 & 40.5 & 3994.9 & 10.8 & 250 \\ 1200 & 37 & 07.7 & 0 & 10.9 & 4020.1 & 10.5 & 247 \\ 1342 & 37 & 00.8 & -0 & 9.8 \mathrm{~W} & 4038.0 & 9.7 & 253 \\ 1528 & 36 & 55.8 & -0 & 30.5 & 4055.2 & 10.9 & 254 \\ 1738 & 36 & 49.2 & -0 & 58.7 & 4078.7 & 10.8 & 250 \\ 1840 & 36 & 45.4 & -1 & 11.8 & 4089.9 & 10.7 & 250 \\ 1920 & 36 & 43.0 & -1 & 20.2 & 4097.0 & 11.1 & 246 \\ 2026 & 36 & 38.1 & -1 & 34.1 & 4109.2 & 10.7 & 248 \\ 2118 & 36 & 34.6 & -1 & 44.8 & 4118.5 & 10.2 & 226 \\ 2208 & 36 & 28.7 & -1 & 52.4 & 4127.0 & 9.8 & 225 \\ 2240 & 36 & 25.0 & -1 & 57.0 & 4132.2 & 10.2 & 260 \\ 2354 & 36 & 22.8 & -2 & 12.4 & 4144.8 & 10.6 & 264\end{array}$

(October 4, 1970)

\begin{tabular}{rrrrrrrr}
138 & 36 & 20.7 & -2 & 35.1 & 4163.2 & 10.1 & 264 \\
330 & 36 & 18.8 & -2 & 58.5 & 4182.1 & 11.4 & 263 \\
444 & 36 & 17.2 & -3 & 15.8 & 4196.2 & 11.2 & 259 \\
533 & 36 & 15.4 & -3 & 27.0 & 4205.4 & 10.6 & 228 \\
549 & 36 & 13.5 & -3 & 29.6 & 4208.2 & 10.7 & 250 \\
632 & 36 & 10.9 & -3 & 38.5 & 4215.9 & 9.8 & 263 \\
816 & 36 & 08.9 & -3 & 59.4 & 4232.9 & 11.4 & 256 \\
855 & 36 & 07.1 & -4 & 08.3 & 4240.3 & 11.3 & 248 \\
918 & 36 & 05.5 & -4 & 13.3 & 4244.6 & 10.7 & 344 \\
920 & 36 & 05.8 & -4 & 13.4 & 4245.0 & 5.3 & 347 \\
1058 & 36 & 14.3 & -4 & 15.8 & 4253.6 & 3.0 & 258 \\
1144 & 36 & 13.8 & -4 & 18.6 & 4255.9 & 10.0 & 257 \\
1254 & 36 & 11.1 & -4 & 32.7 & 4267.6 & 10.5 & 257 \\
1330 & 36 & 09.7 & -4 & 40.3 & 4273.9 & 11.1 & 258 \\
1442 & 36 & 07.0 & -4 & 56.4 & 4287.2 & 10.5 & 259 \\
1535 & 36 & 05.2 & -5 & 07.7 & 4296.5 & 11.2 & 249 \\
\hline
\end{tabular}


TABLE 1 - Continued

\begin{tabular}{|c|c|c|c|c|c|c|c|}
\hline \multirow{2}{*}{$\frac{\text { Time }}{1550}$} & \multicolumn{2}{|c|}{ Latitude } & \multicolumn{2}{|c|}{ Longitude } & \multirow{2}{*}{$\begin{array}{c}\begin{array}{c}\text { Distance } \\
\text { (miles) }\end{array} \\
4299.4\end{array}$} & \multirow{2}{*}{$\begin{array}{c}\begin{array}{c}\text { Speed } \\
\text { (knots) }\end{array} \\
9.7\end{array}$} & \multirow{2}{*}{$\begin{array}{c}\begin{array}{c}\text { Course } \\
\text { (degrees) }\end{array} \\
251\end{array}$} \\
\hline & 36 & 04.2 & -5 & 11.0 & & & \\
\hline 1627 & 36 & 02.2 & -5 & 18.0 & 4305.4 & 10.4 & 250 \\
\hline 1720 & 35 & 59.1 & -5 & 28.7 & 4314.6 & 9.2 & 259 \\
\hline 1812 & 35 & 57.6 & -5 & 38.4 & 4322.5 & 9.5 & 285 \\
\hline 1855 & 35 & 59.4 & -5 & 46.5 & 4329.3 & 9.9 & 291 \\
\hline 1937 & 36 & 01.9 & -5 & 54.5 & 4336.3 & 9.3 & 289 \\
\hline 2035 & 36 & 04.9 & -6 & 05.0 & 4345.3 & 9.8 & 282 \\
\hline 2108 & 36 & 06.0 & -6 & 11.5 & 4350.6 & 10.6 & 296 \\
\hline 2122 & 36 & 07.1 & -6 & 14.2 & 4353.1 & 8.9 & 298 \\
\hline 2144 & 36 & 08.6 & -6 & 17.8 & 4356.4 & 10.7 & 292 \\
\hline 2300 & 36 & 13.6 & -6 & 33.4 & 4369.9 & 9.7 & 291 \\
\hline
\end{tabular}

(October 5, 1970)

\begin{tabular}{rrrrrrrr}
044 & 36 & 19.5 & -6 & 52.8 & 4386.7 & 10.2 & 288 \\
054 & 36 & 20.1 & -6 & 54.9 & 4388.4 & 10.0 & 290 \\
236 & 36 & 25.9 & -7 & 14.7 & 4405.4 & 10.7 & 289 \\
426 & 36 & 32.3 & -7 & 37.7 & 4425.0 & 9.3 & 290 \\
524 & 36 & 35.4 & -7 & 48.2 & 4433.0 & 9.7 & 327 \\
535 & 36 & 36.9 & -7 & 49.4 & 4435.7 & 8.9 & 295 \\
542 & 36 & 37.3 & -7 & 50.6 & 4436.8 & 10.1 & 290 \\
726 & 36 & 43.3 & -8 & 11.0 & 4454.2 & 9.5 & 288 \\
922 & 36 & 48.8 & -8 & 33.0 & 4472.6 & 9.6 & 292 \\
1042 & 36 & 53.7 & -8 & 47.8 & 4485.5 & 9.4 & 291 \\
1132 & 36 & 56.5 & -8 & 57.0 & 4493.3 & 9.9 & 310 \\
1158 & 36 & 59.3 & -9 & 01.1 & 4497.6 & 8.7 & 325 \\
1215 & 37 & 01.3 & -9 & 02.9 & 4500.1 & 9.5 & 354 \\
1234 & 37 & 04.3 & -9 & 03.3 & 4503.1 & 9.6 & 345 \\
1354 & 37 & 16.7 & -9 & 07.4 & 4515.9 & 9.1 & 348 \\
1436 & 37 & 22.9 & -9 & 09.0 & 4522.3 & 8.8 & 346 \\
1536 & 37 & 31.5 & -9 & 11.6 & 4531.1 & 9.1 & 349 \\
1628 & 37 & 39.3 & -9 & 13.5 & 4539.0 & 8.8 & 351 \\
1744 & 37 & 50.3 & -9 & 15.6 & 4550.2 & 10.0 & 343 \\
1814 & 37 & 55.1 & -9 & 17.4 & 4555.2 & 9.0 & 345 \\
1928 & 38 & 05.8 & -9 & 21.0 & 4566.3 & 8.7 & 349 \\
2028 & 38 & 14.4 & -9 & 23.0 & 4575.0 & 9.2 & 350 \\
2045 & 38 & 17.0 & -9 & 23.6 & 4577.6 & 9.5 & 321 \\
2142 & 38 & 24.0 & -9 & 30.9 & 4586.7 & 9.7 & 236 \\
2206 & 38 & 21.8 & -9 & 35.0 & 4590.6 & 9.8 & 225 \\
2216 & 38 & 20.7 & -9 & 36.5 & 4592.2 & 8.9 & 140 \\
2304 & 38 & 15.2 & -9 & 30.7 & 4599.3 & 9.8 & 145 \\
2328 & 38 & 12.0 & -9 & 27.8 & 4603.3 & 11.3 & 225 \\
2348 & 38 & 09.3 & -9 & 31.2 & 4607.0 & 9.5 & 233 \\
\hline
\end{tabular}

TABLE 1 - Continued

\begin{tabular}{|c|c|c|c|c|c|c|c|}
\hline \multirow[t]{2}{*}{ Time } & \multicolumn{2}{|c|}{ Latitude } & \multicolumn{2}{|c|}{ Longitude } & $\begin{array}{c}\text { Distance } \\
\text { (miles) }\end{array}$ & $\begin{array}{c}\text { Speed } \\
\text { (knots) }\end{array}$ & $\begin{array}{c}\text { Course } \\
\text { (degrees) }\end{array}$ \\
\hline & & & \multicolumn{5}{|c|}{ (October 6, 1970) } \\
\hline 003 & 38 & 07.9 & -9 & 33.6 & 4609.4 & 8.9 & 63 \\
\hline 006 & 38 & 08.1 & -9 & 33.1 & 4609.8 & 6.4 & 339 \\
\hline 010 & 38 & 08.5 & -9 & 33.3 & 4610.3 & 9.0 & 312 \\
\hline 015 & 38 & 09.0 & -9 & 34.0 & 4611.0 & 9.3 & 321 \\
\hline 103 & 38 & 14.8 & -9 & 39.9 & 4618.4 & 7.3 & 21 \\
\hline 150 & 38 & 20.2 & -9 & 37.2 & 4624.2 & 12.0 & 22 \\
\hline 250 & 38 & 31.3 & -9 & 31.5 & 4636.2 & 11.4 & 33 \\
\hline 345 & 38 & 40.0 & -9 & 24.2 & 4646.6 & & \\
\hline
\end{tabular}

\section{ARRIVE LISBON}

50 feet, towed $\approx 300$ meters behind the vessel; (3) a Bolt PA6 amplifier and filter module; (4) two Edo Western Model 333 Precision Bathymetric Recorders displaying on dry Timefax paper. One recorder was set on a 10-second sweep, the other on a 5-second sweep. The 10-second sweep records are annotated and shown in Figures 3, A, B, C, etc.

\section{ACKNOWLEDGMENTS}

The scientific party of Leg 13 is extremely grateful to Captain Joseph A. Clarke, his officers and crew for their excellence in navigation and cooperative spirit. Thanks goes also to the Scripps group for the many hours of watch standing and their patience with the scientific team during the high-tension moments of arriving on site. The digital processing of the navigation, bathymetry, and magnetic data was undertaken at the Lamont-Doherty Geological Observatory of Columbia University with the help of Cheryl McDonald and Edward Haff. L.D.G.O. Contribution No. 1855 .

\section{REFERENCE}

Talwani, M., 1969. A computer system for the reduction, storage and display of underway data acquired at sea. Technical Report 1, Contract CU-1-69 N0014-67-A0108-0004, Lamont-Doherty Geological Observatory of Columbia University, Palisades, New York, 1. 


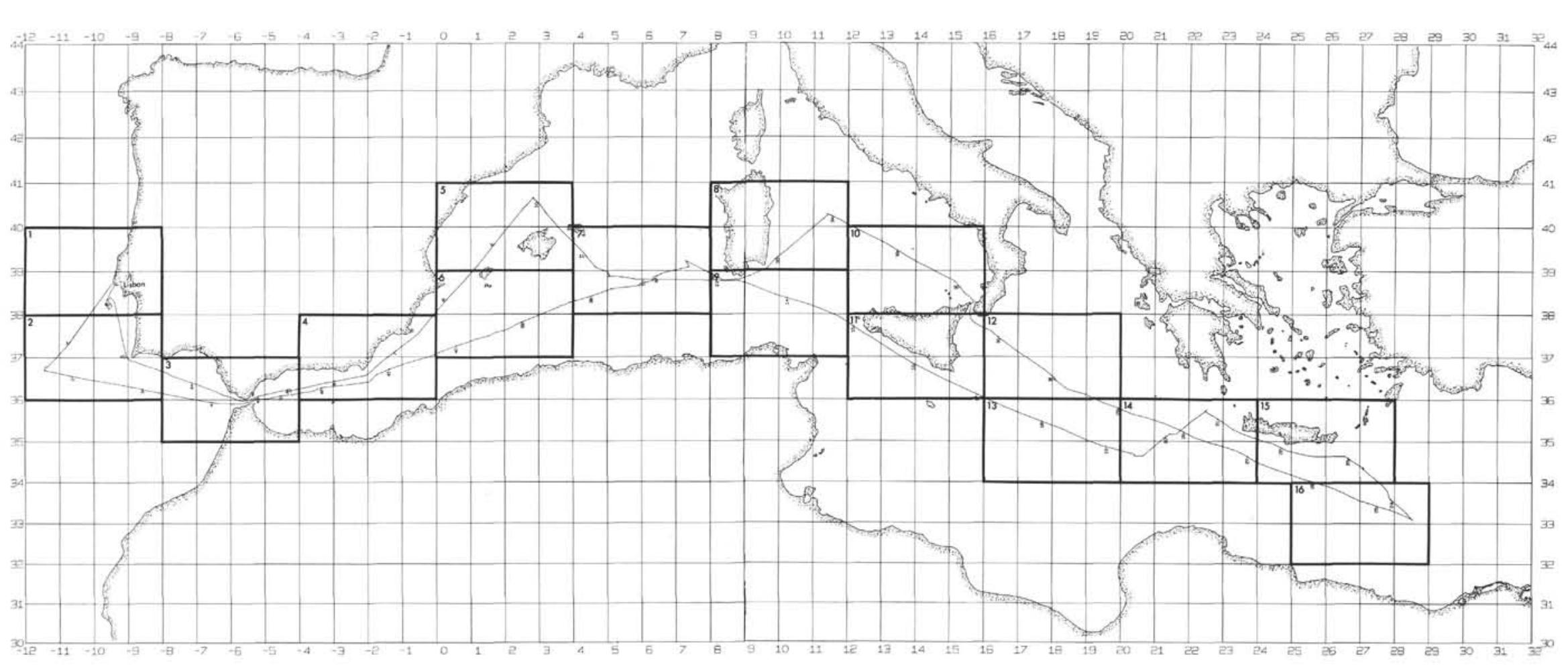

Figure 1A. Navigation and bathymetry, mercator projection. 


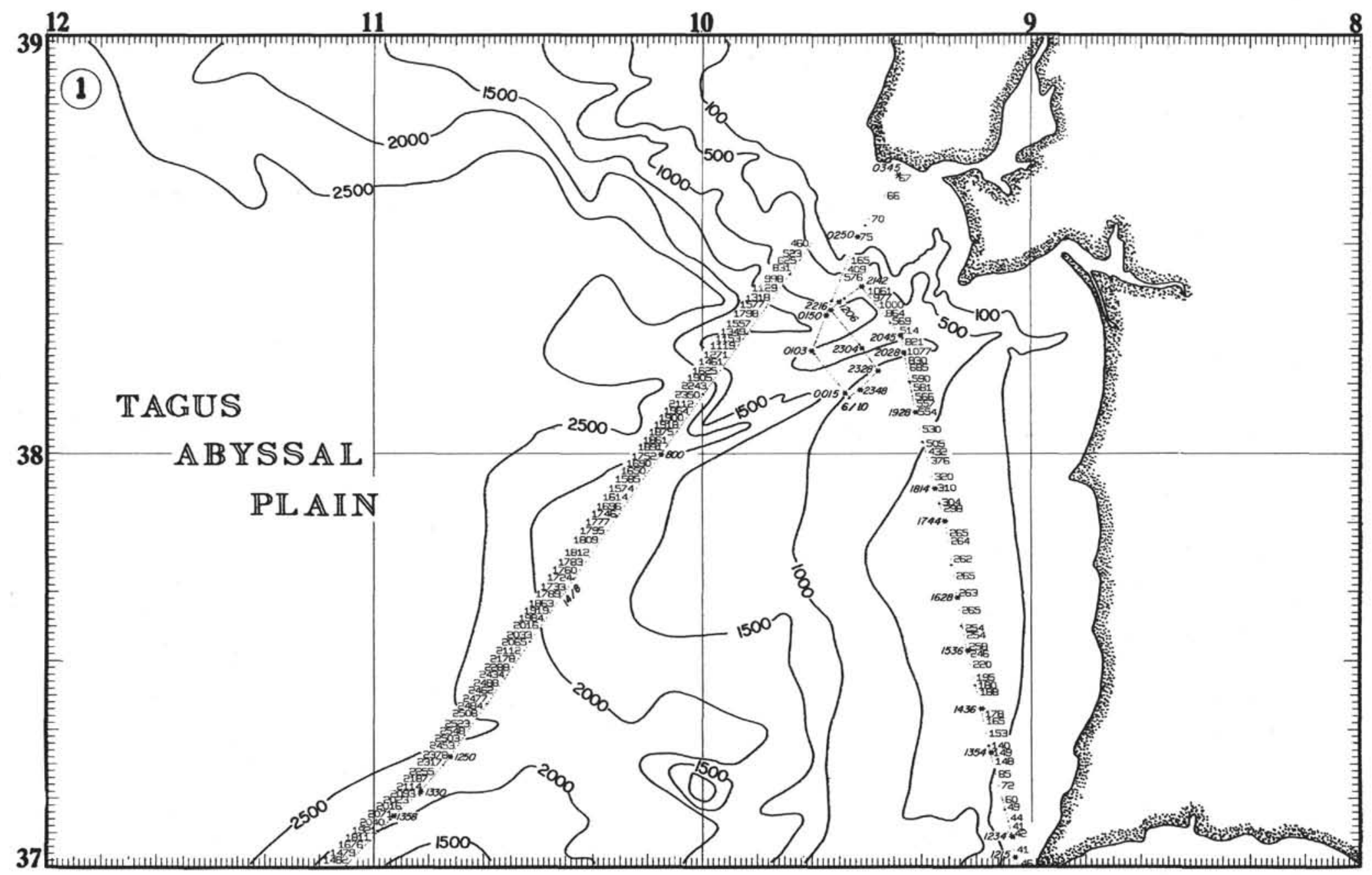

Figure 1B. 


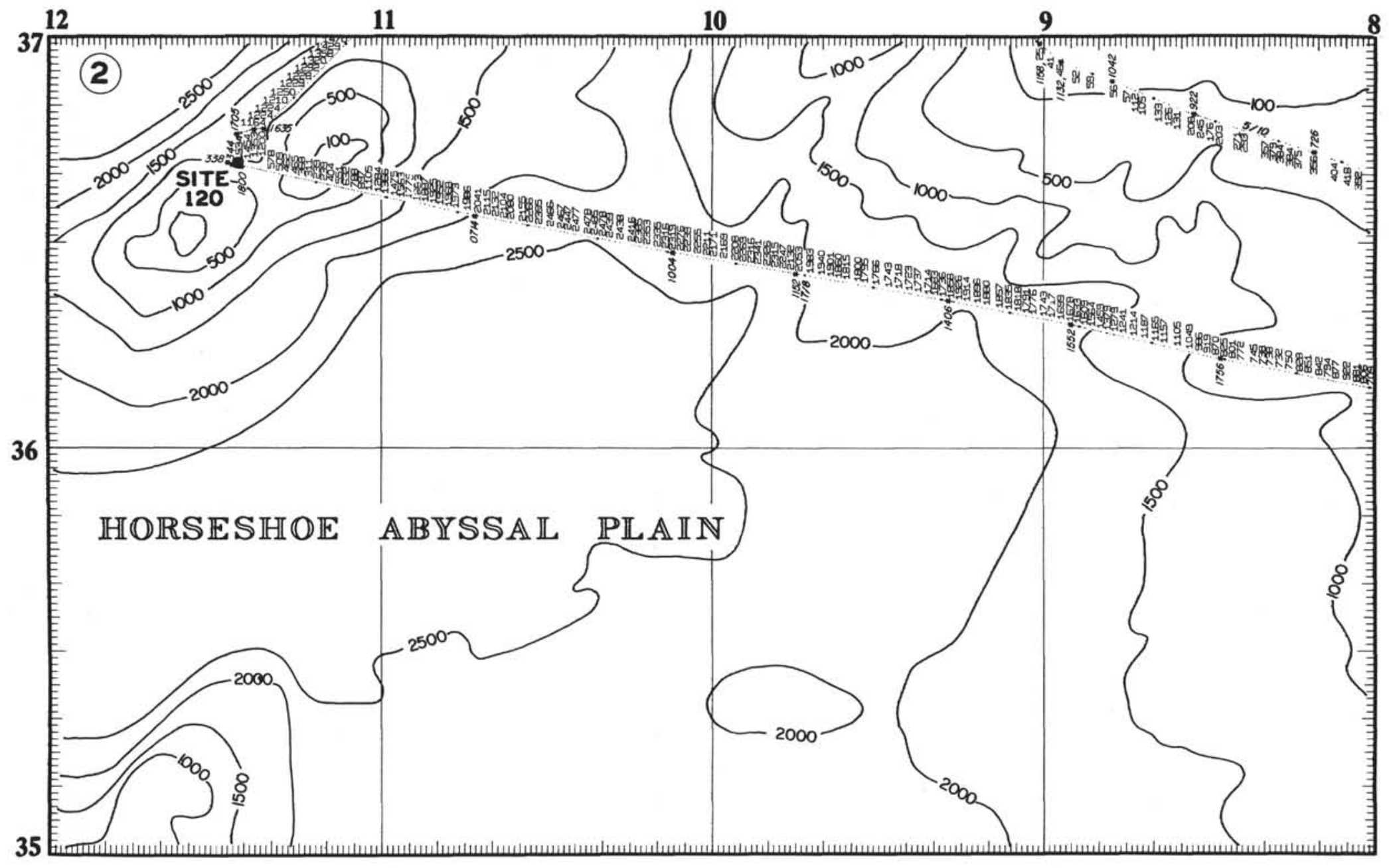

Figure 1C.

Depths in units of travel time $(1$ tau $=1 / 400$ th sec $)$. 


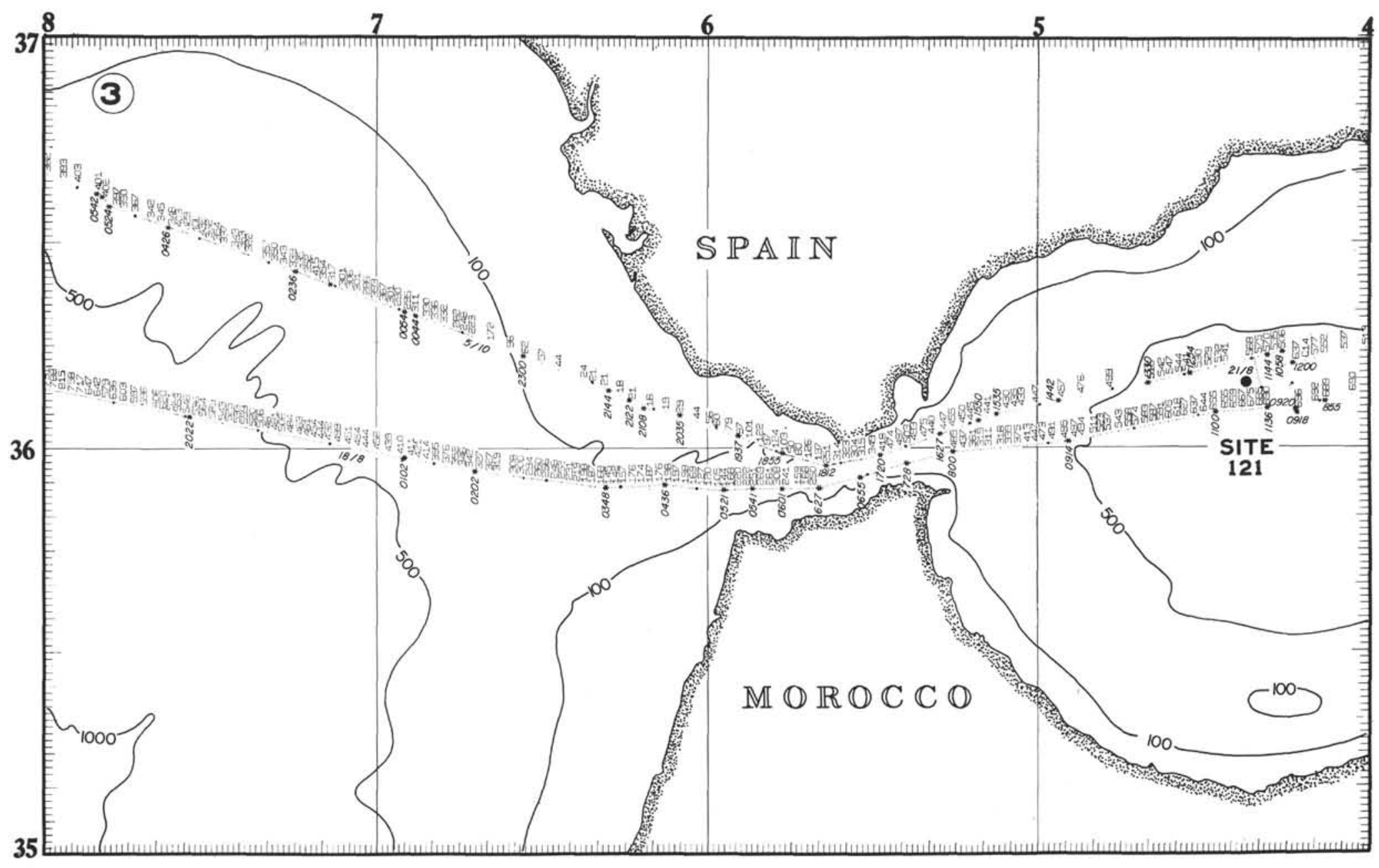

Figure 1D. 


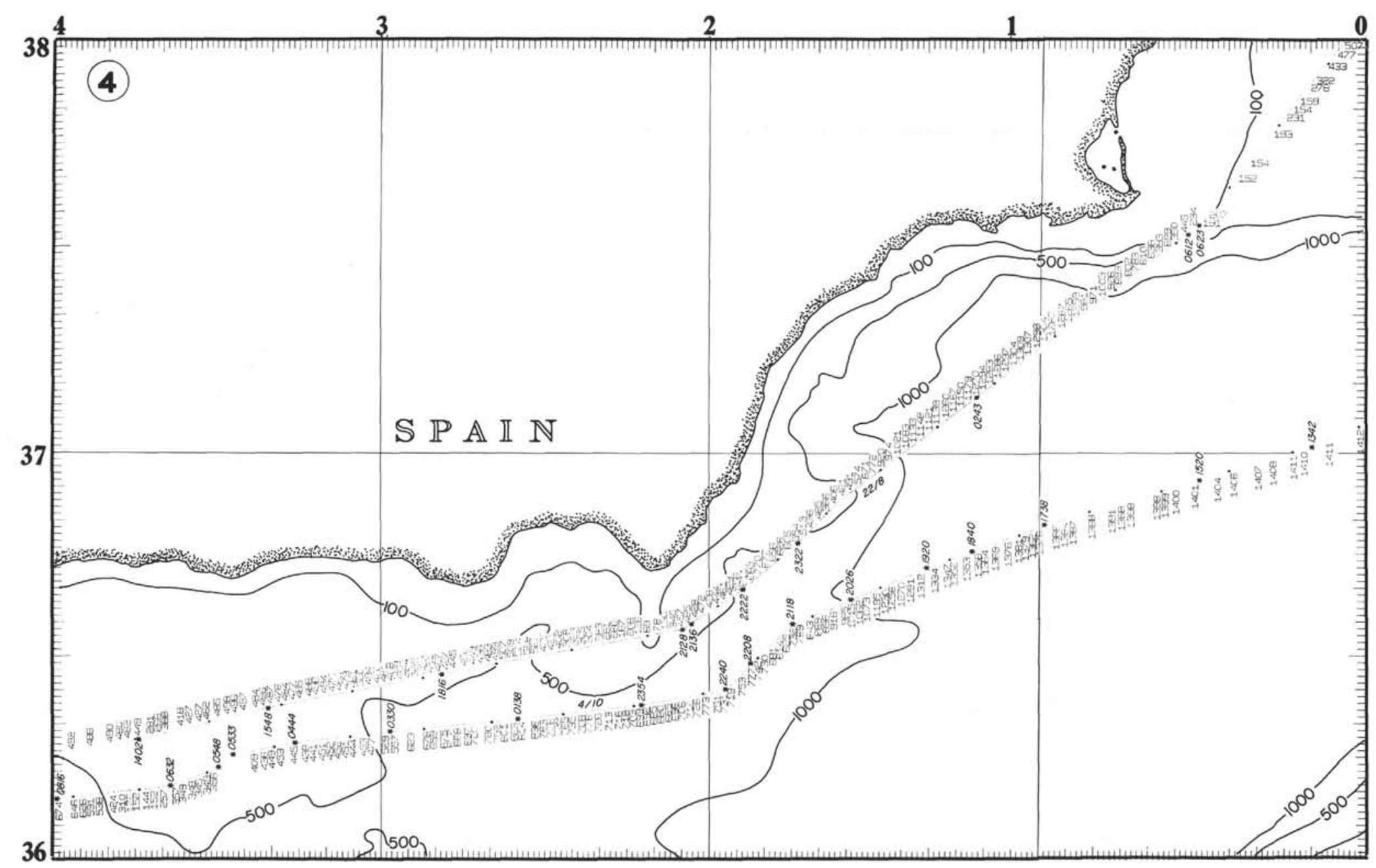

Figure 1E.

Depths in units of travel time ( 1 tau $=1 / 400$ th sec $)$. 


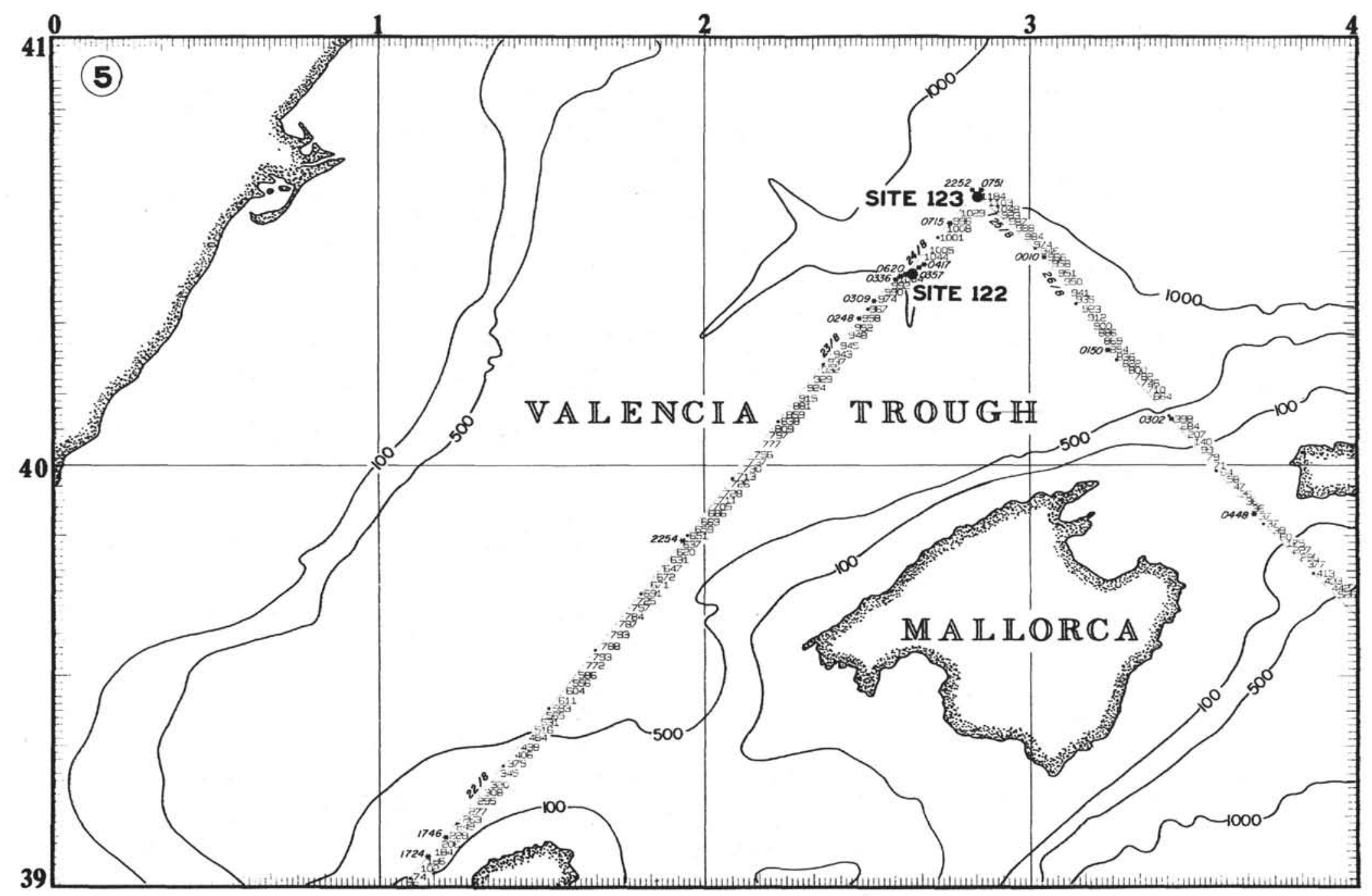

Figure 1F. 


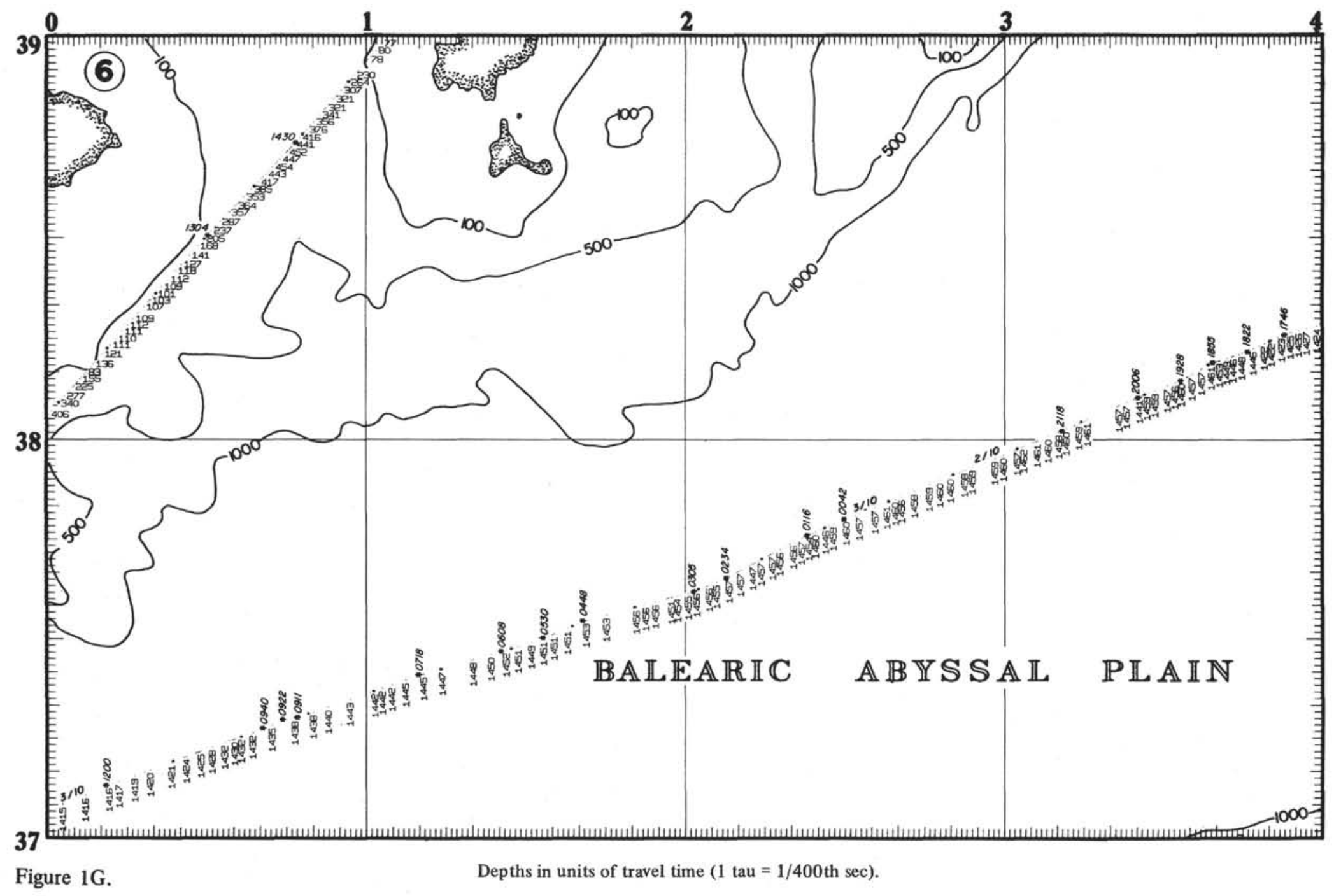

స్త 


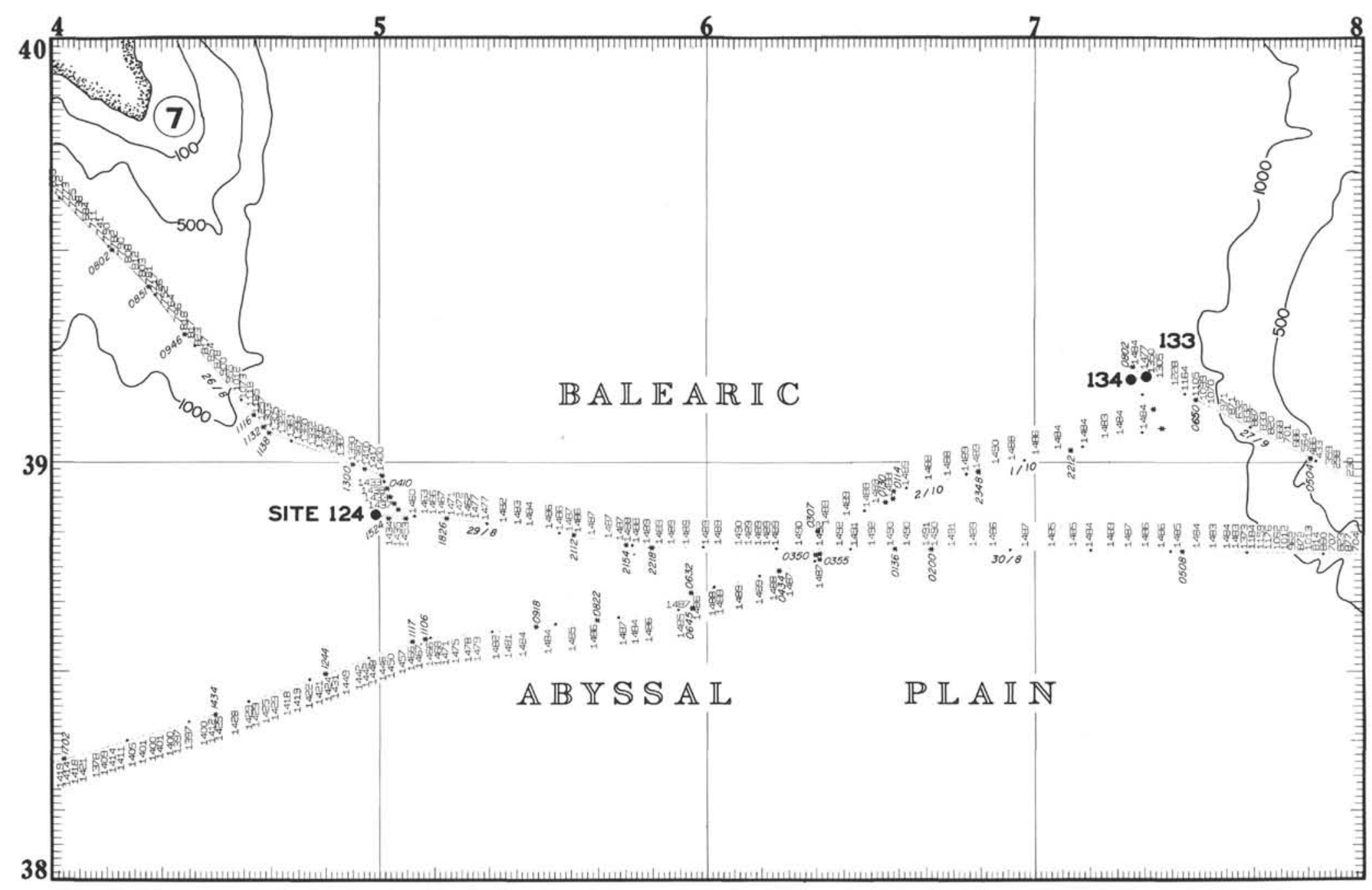

Figure $1 \mathrm{H}$. 


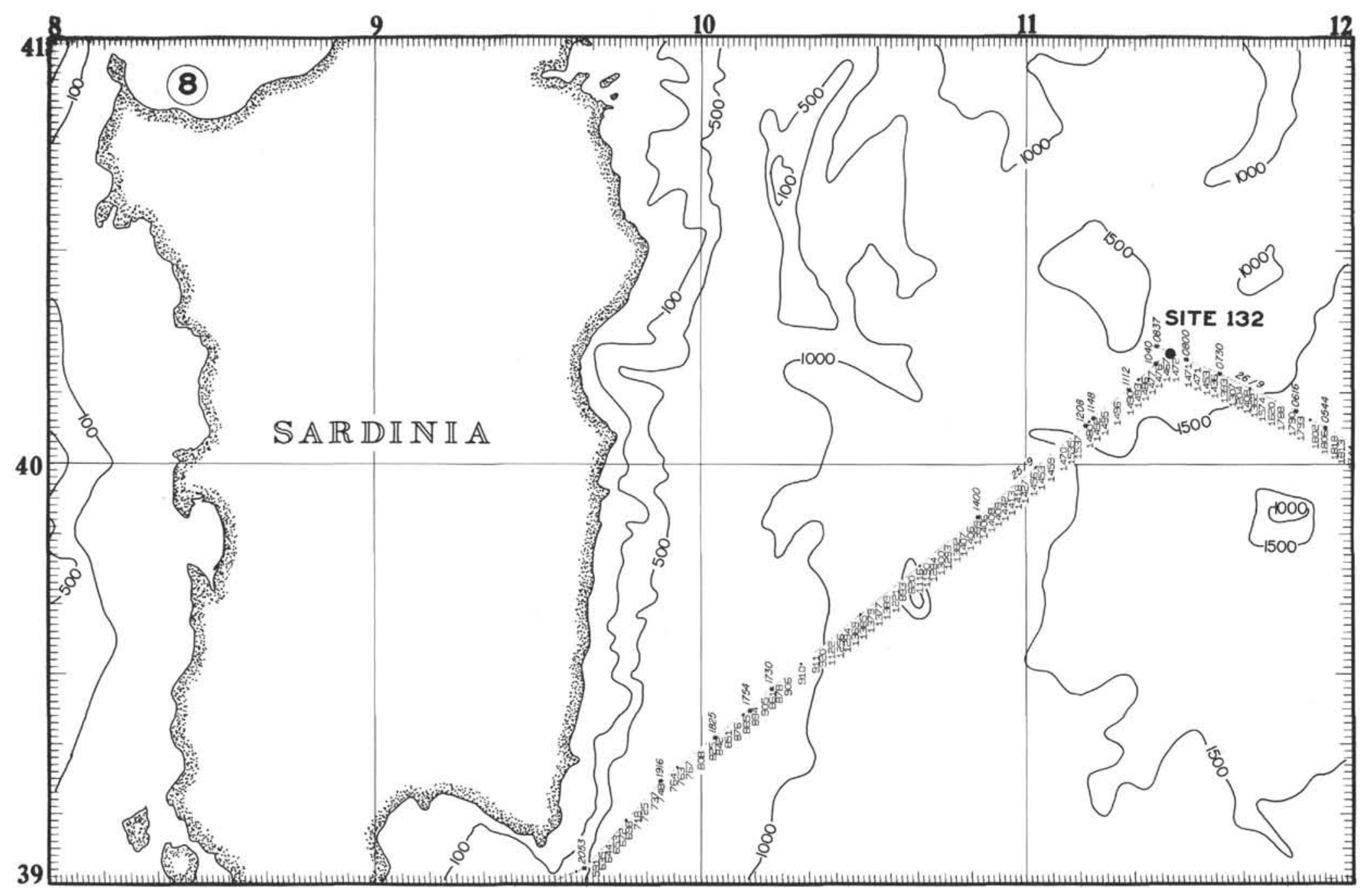

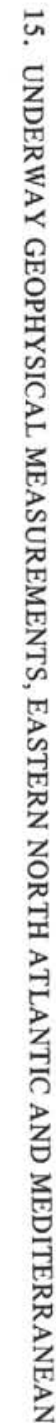




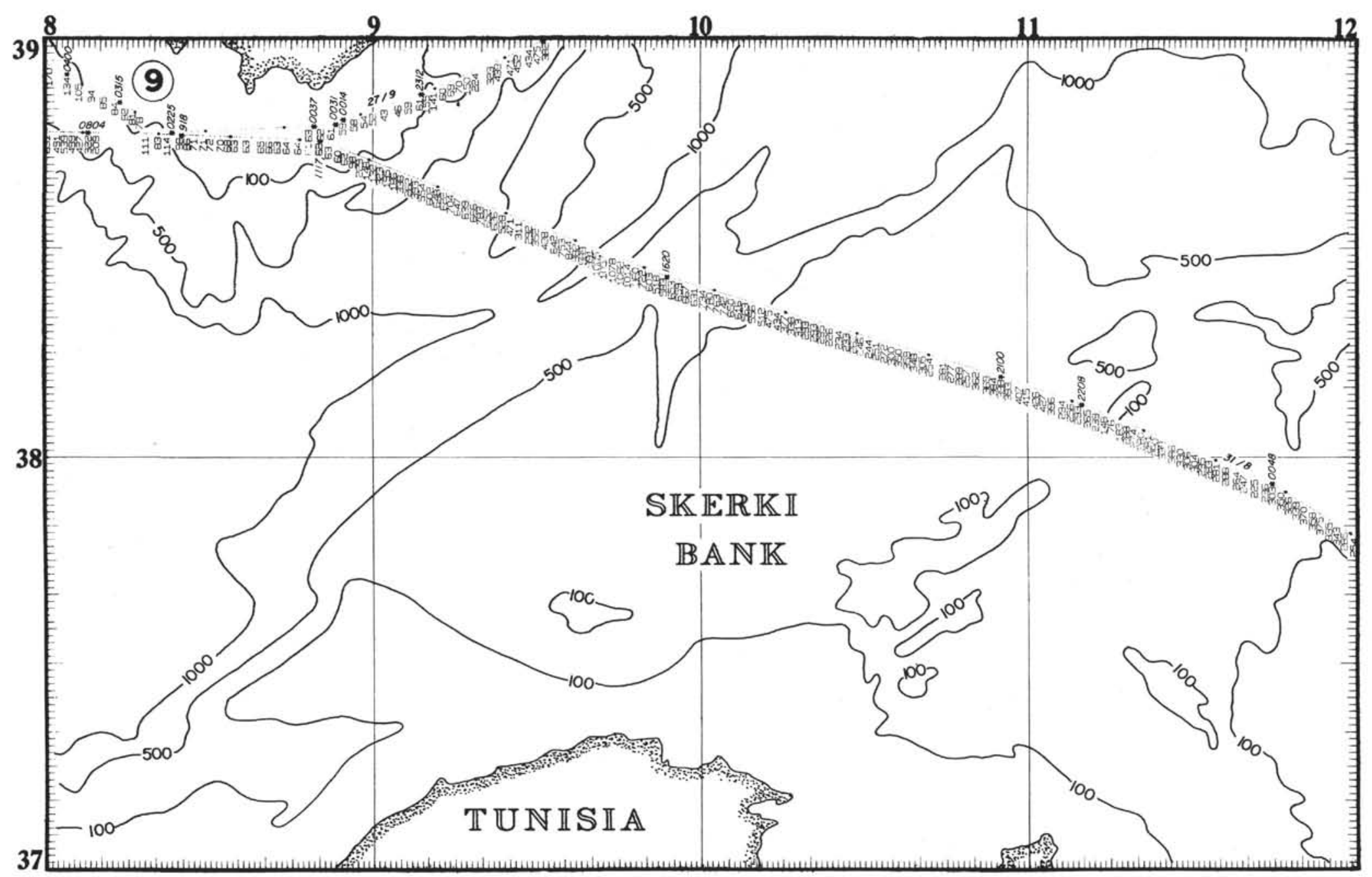

Figure 1J. 


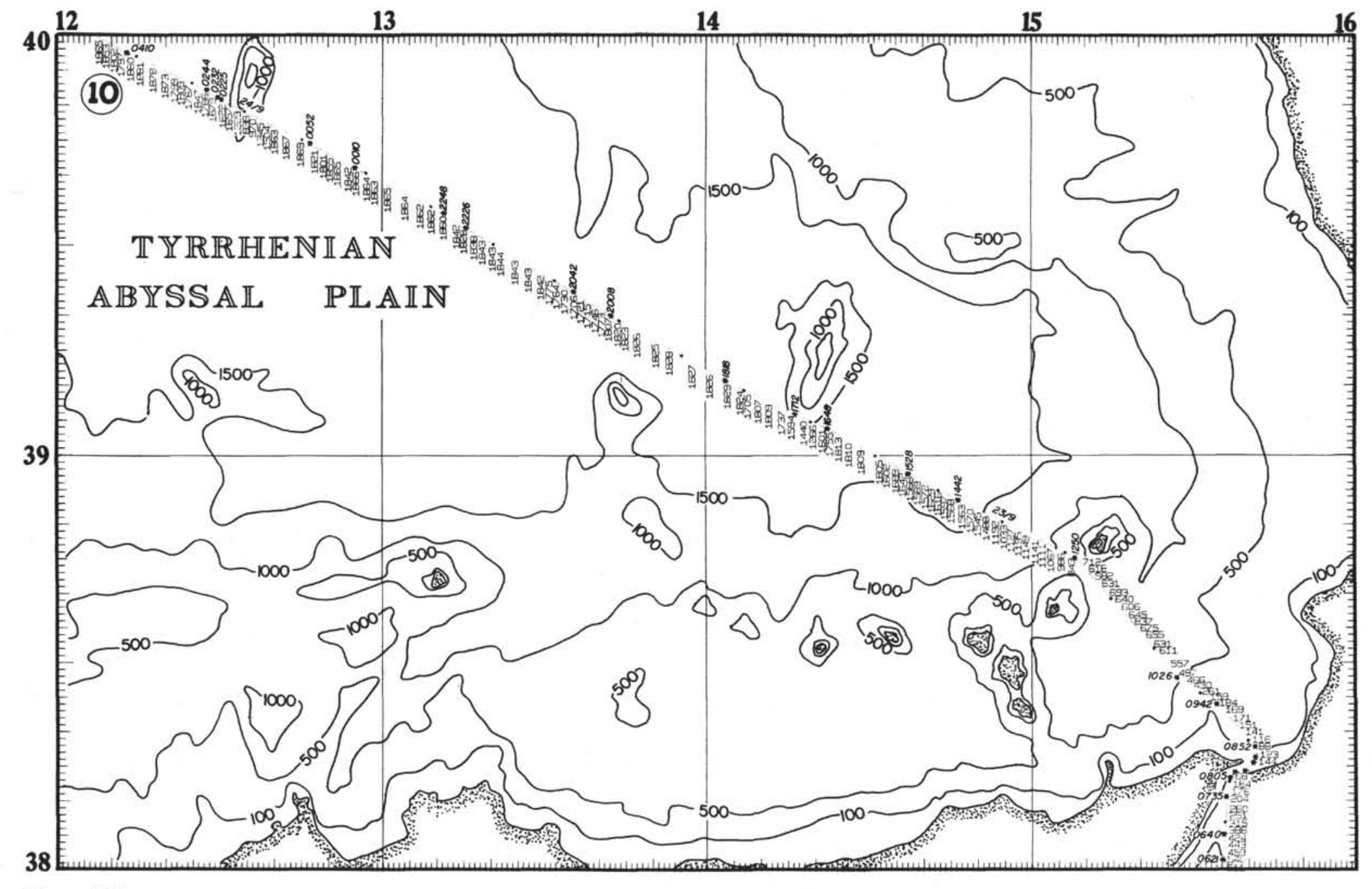

Figure 1K.

Depths in units of travel time ( $(1$ tau $=1 / 400$ th sec $)$. 


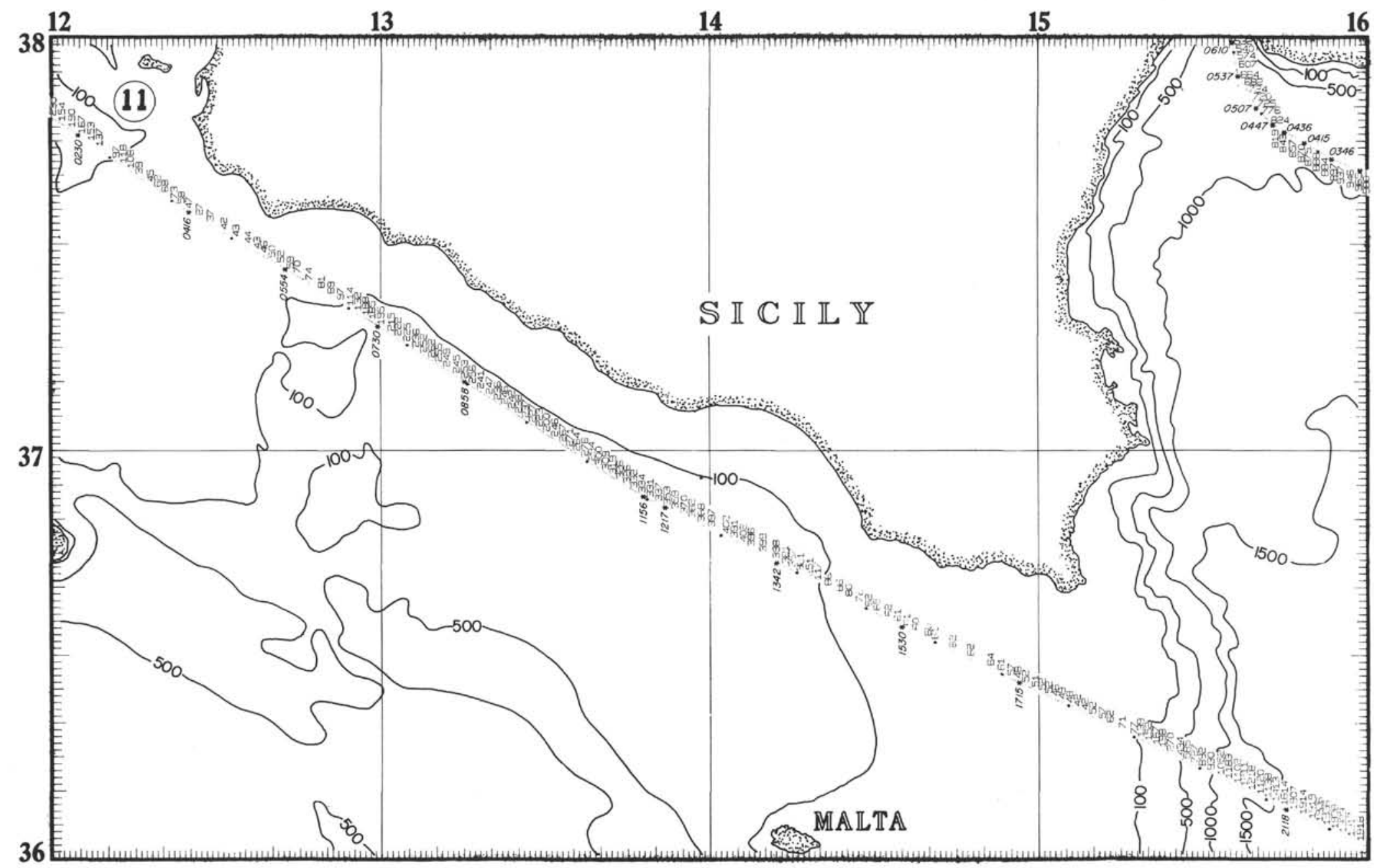




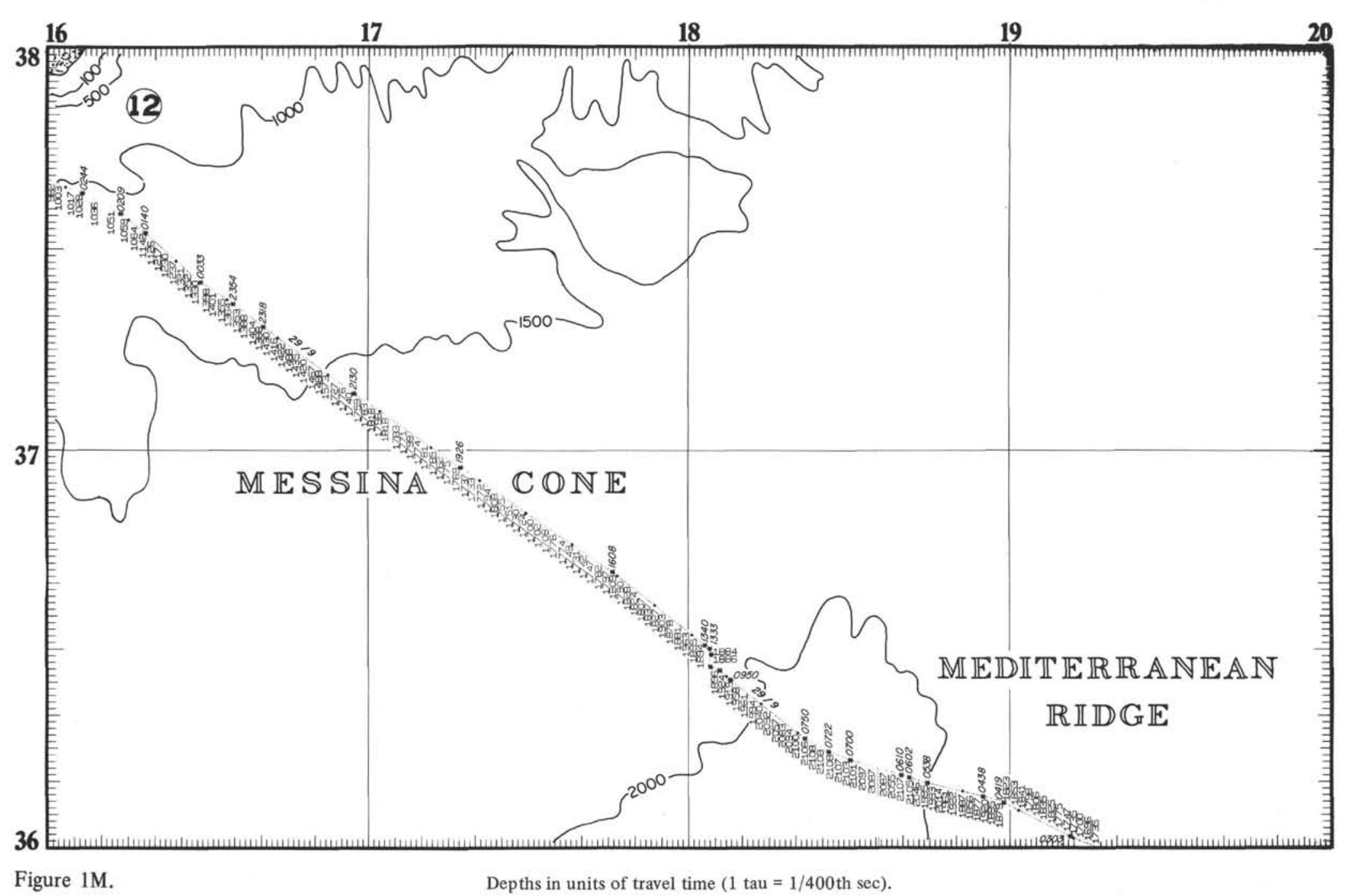

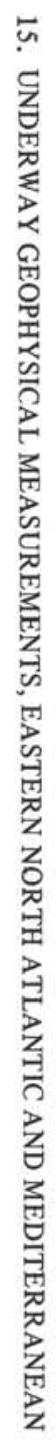




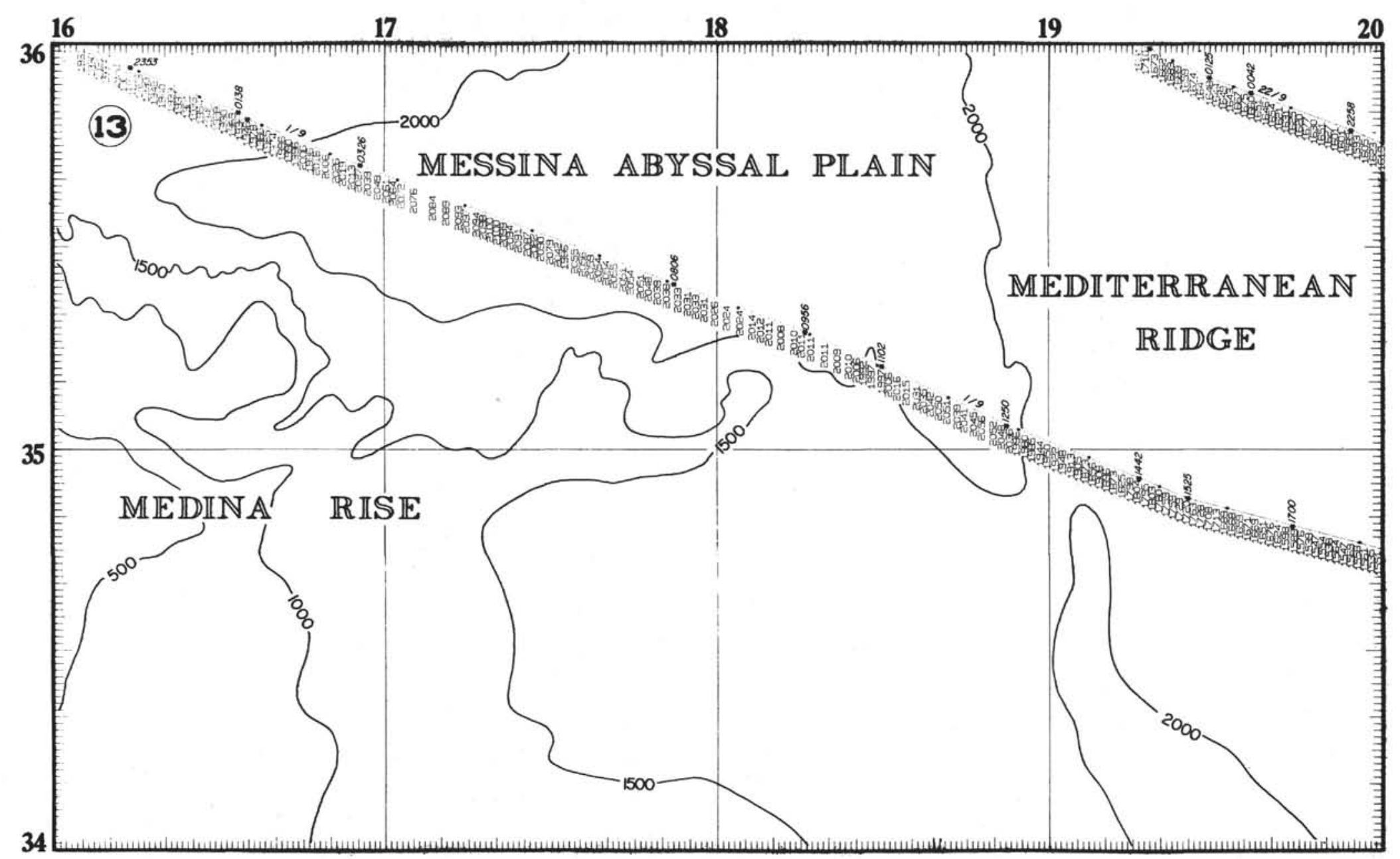

Figure 1N. 


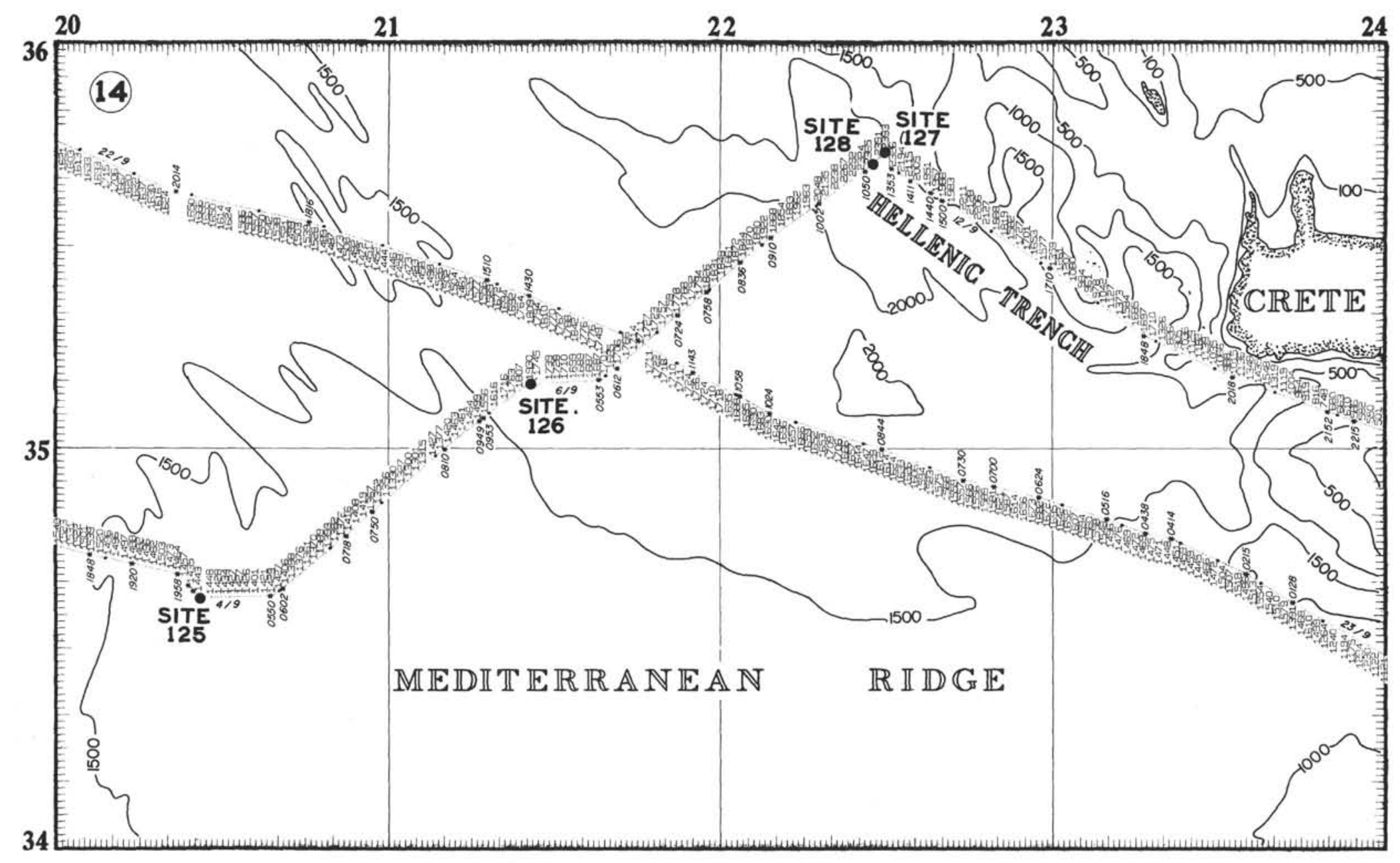

Figure 10.

Depths in units of travel time ( 1 tau $=1 / 400$ th sec $)$ 


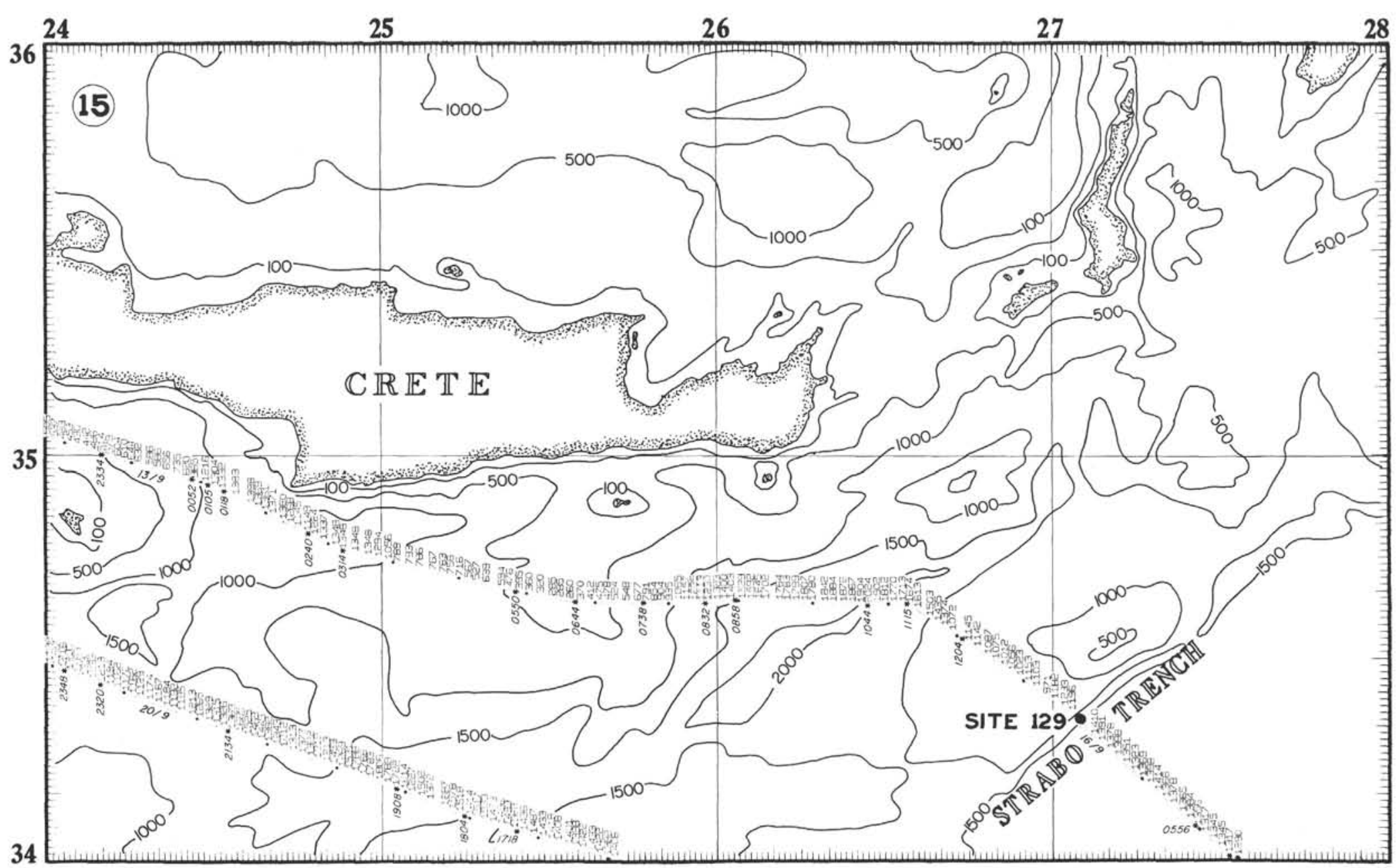

Figure 1P. 


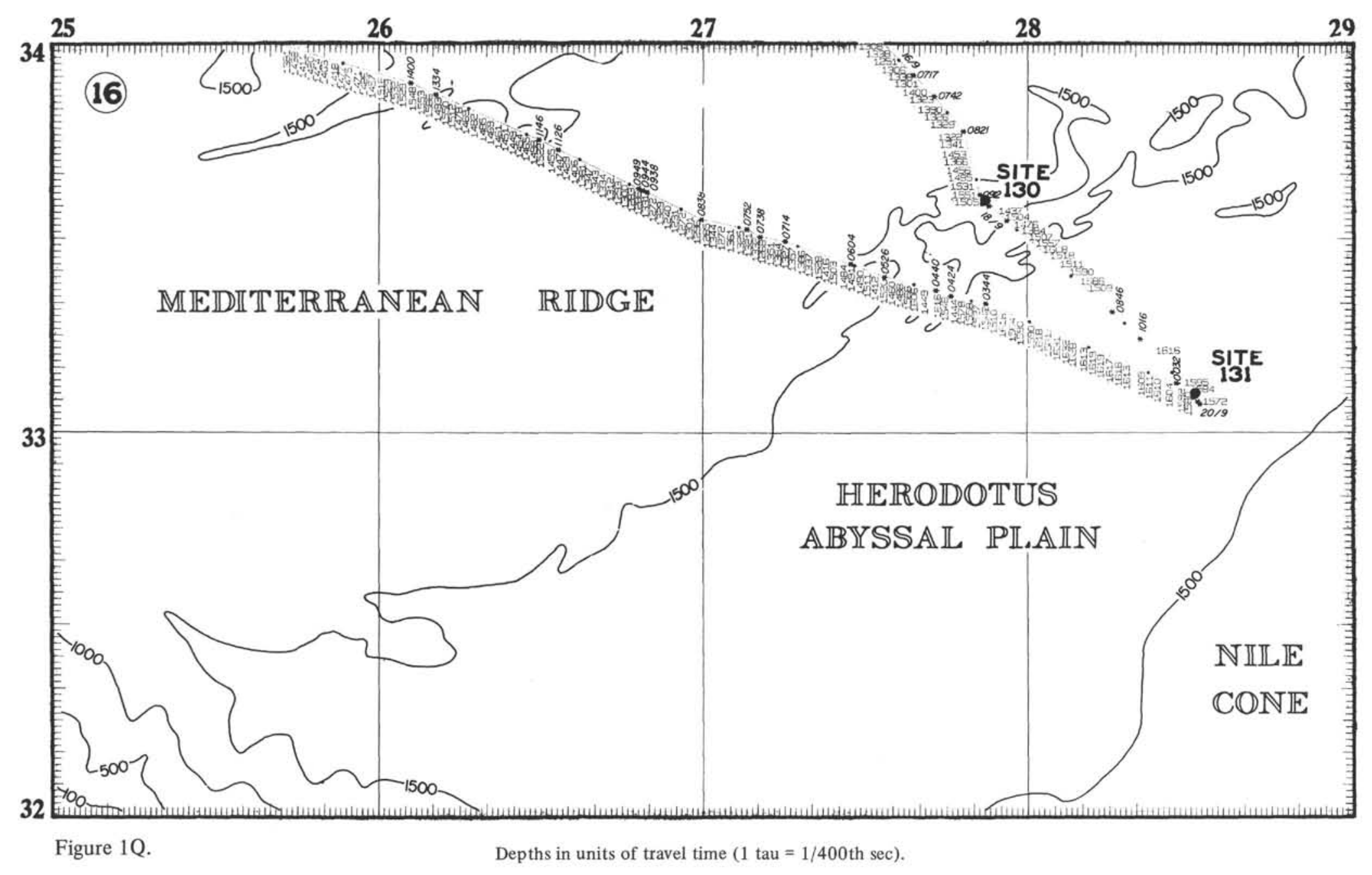

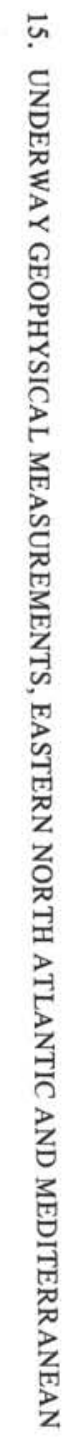


W. B. F. RYAN, T. B. GUSTAFSON

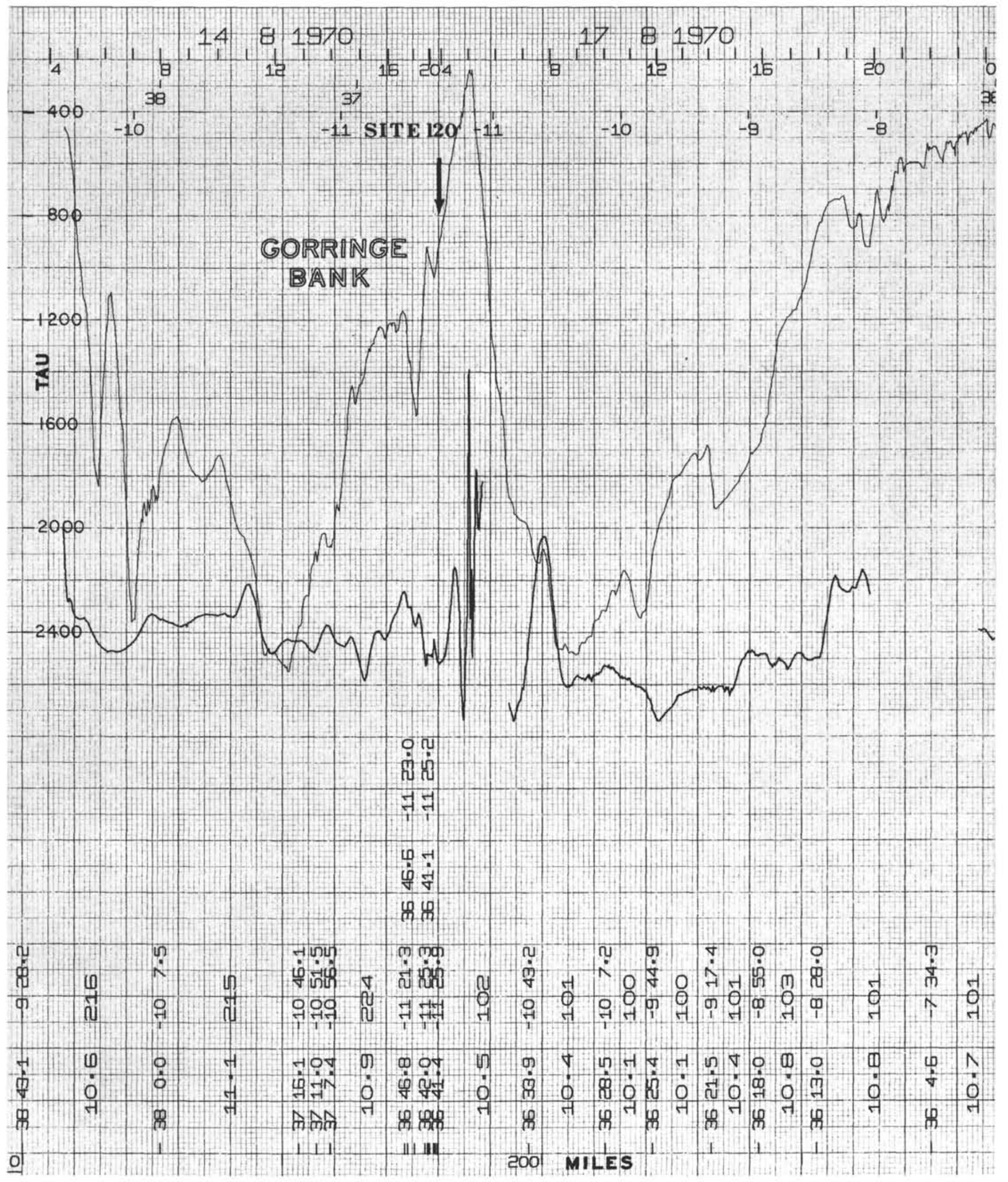

Figure 2A. Topographic and magnetic profiles. 


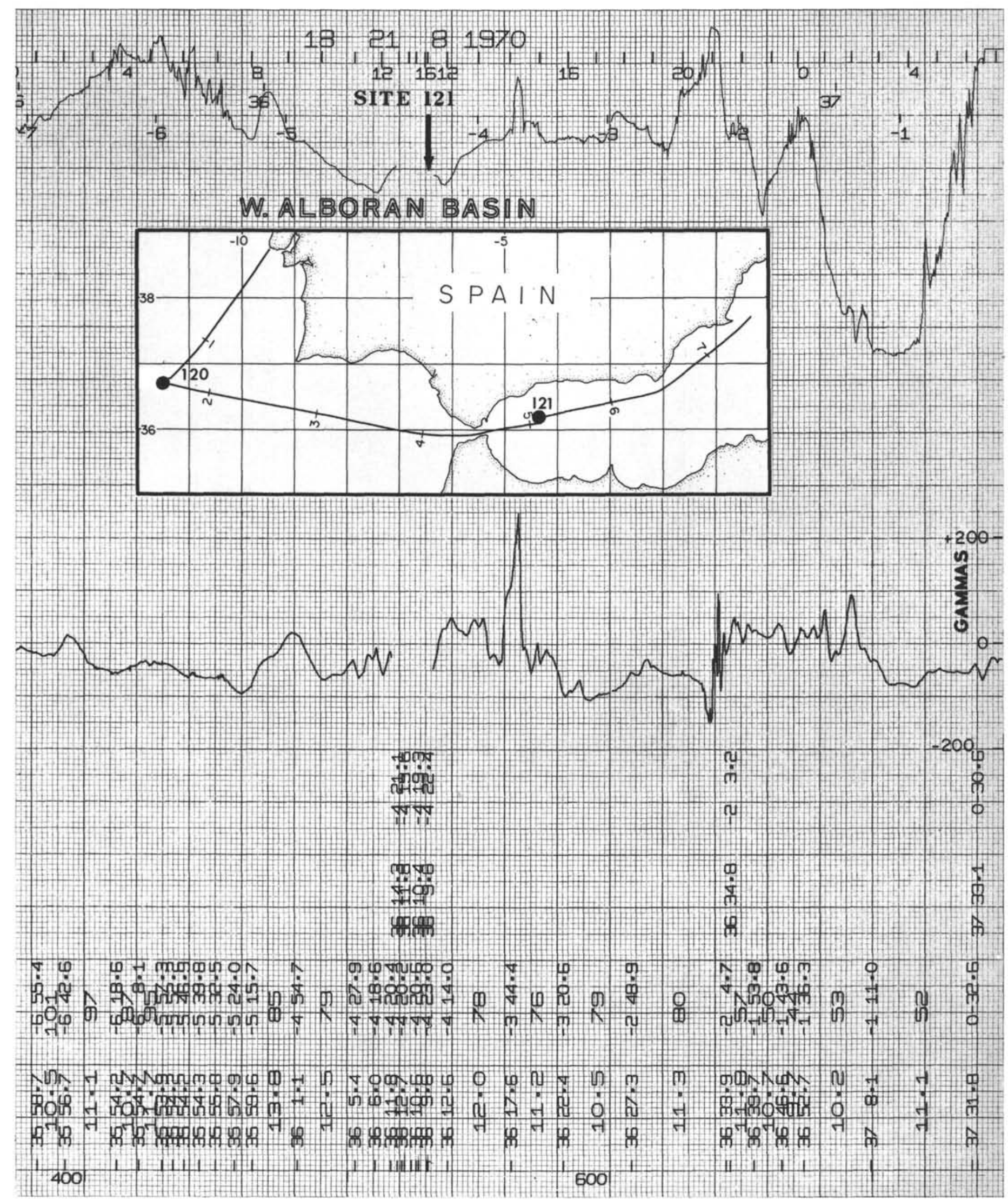

Figure 2B. 


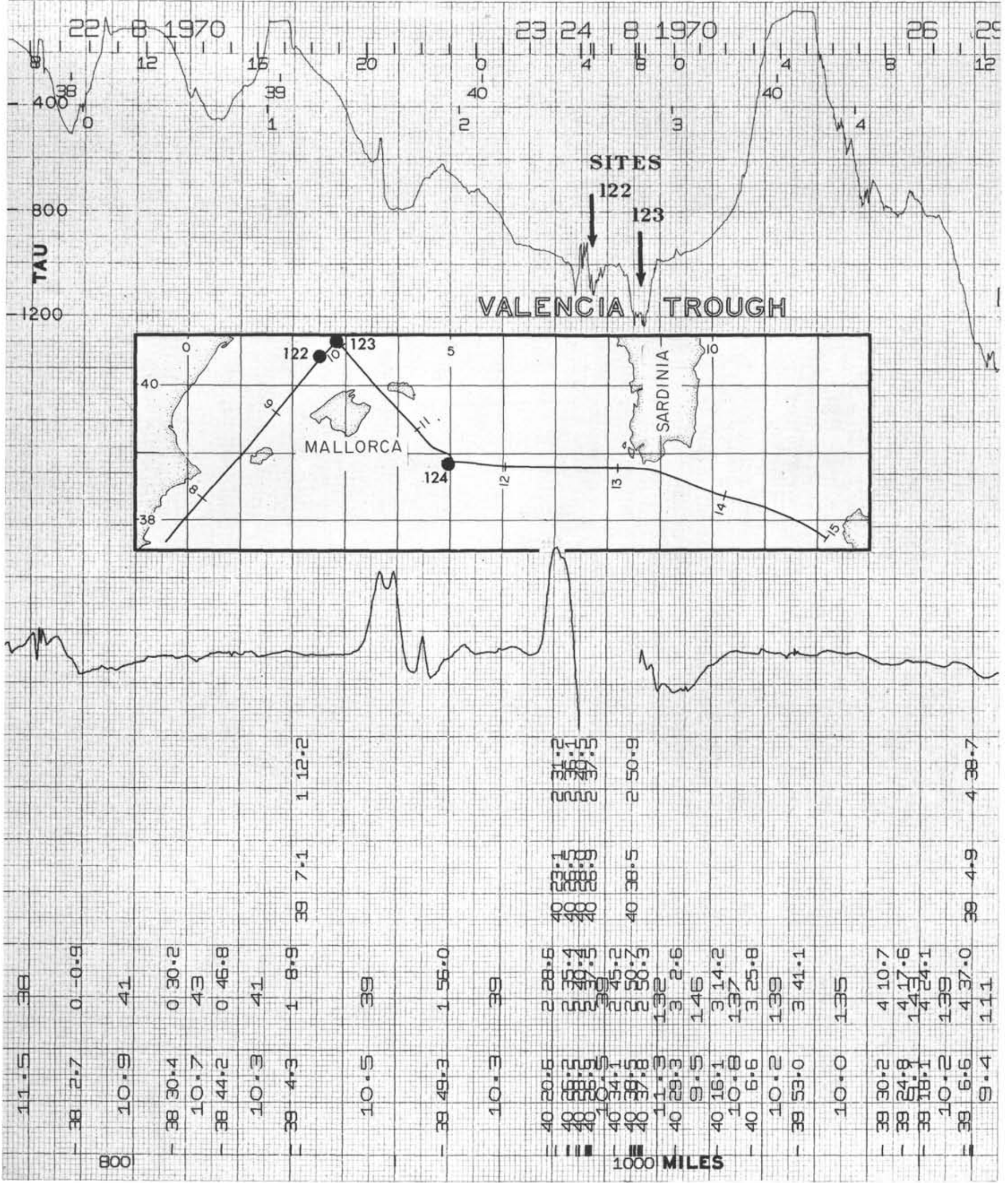

Figure 2C. 


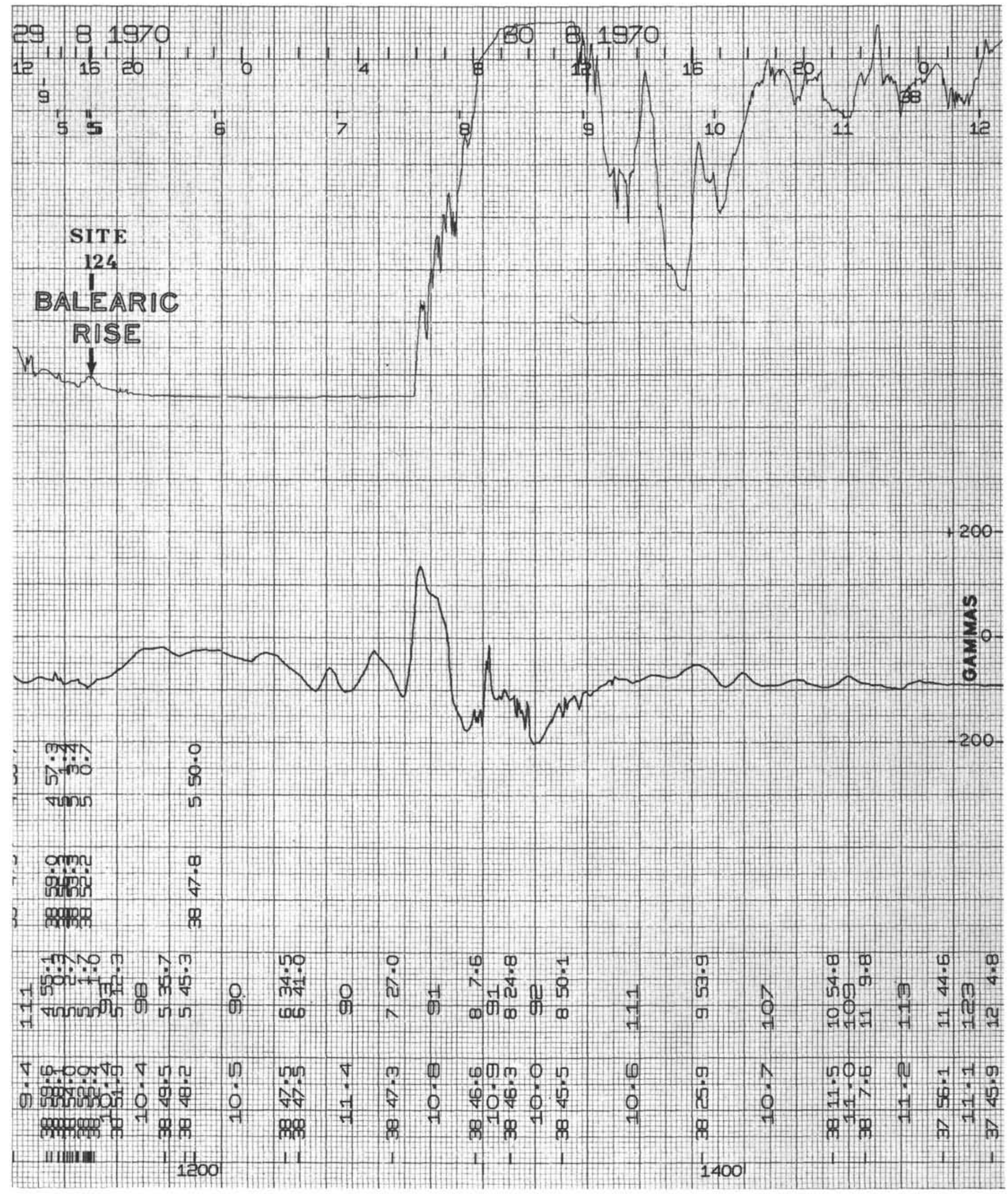

Figure 2D. 

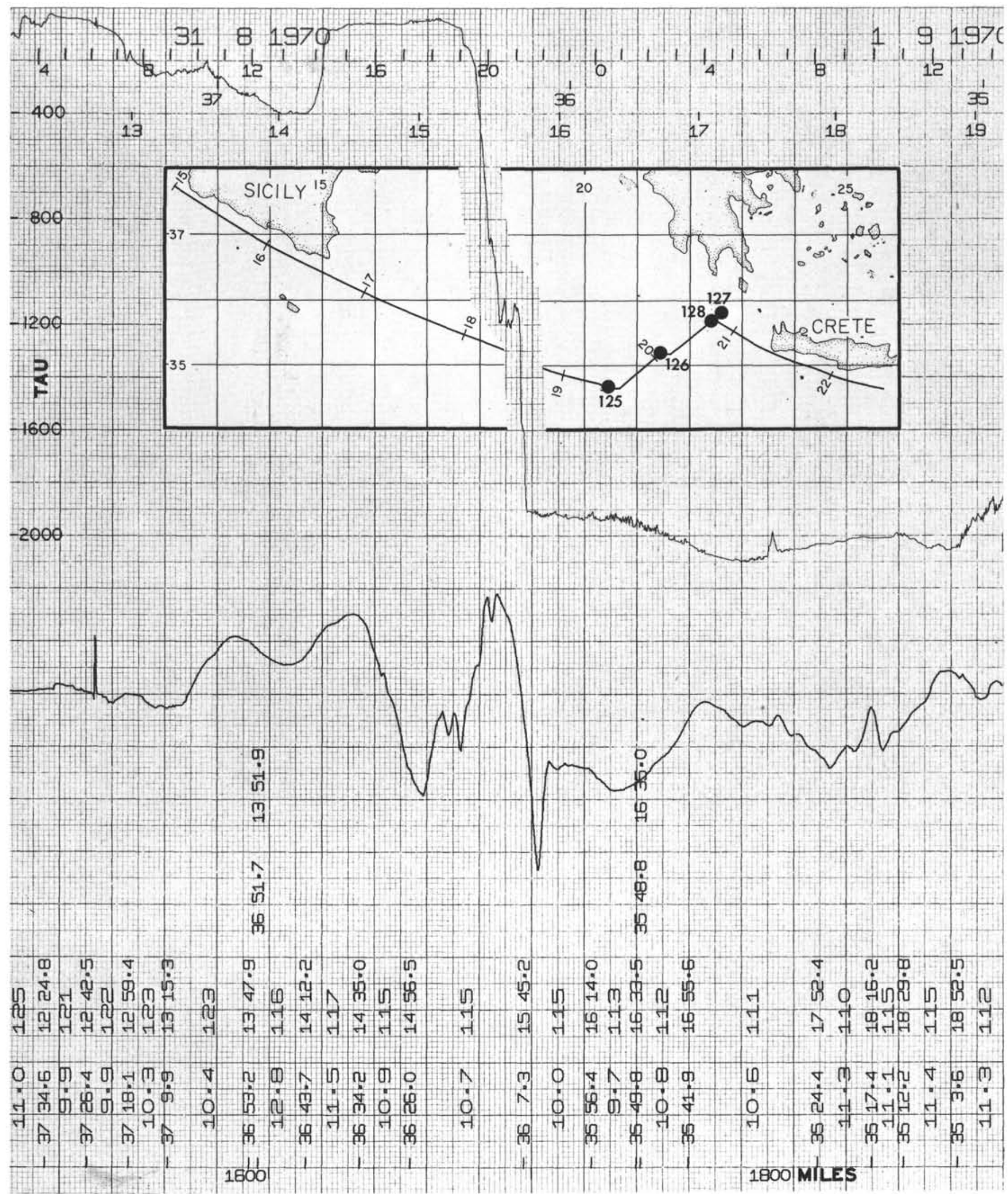

Figure 2E. 


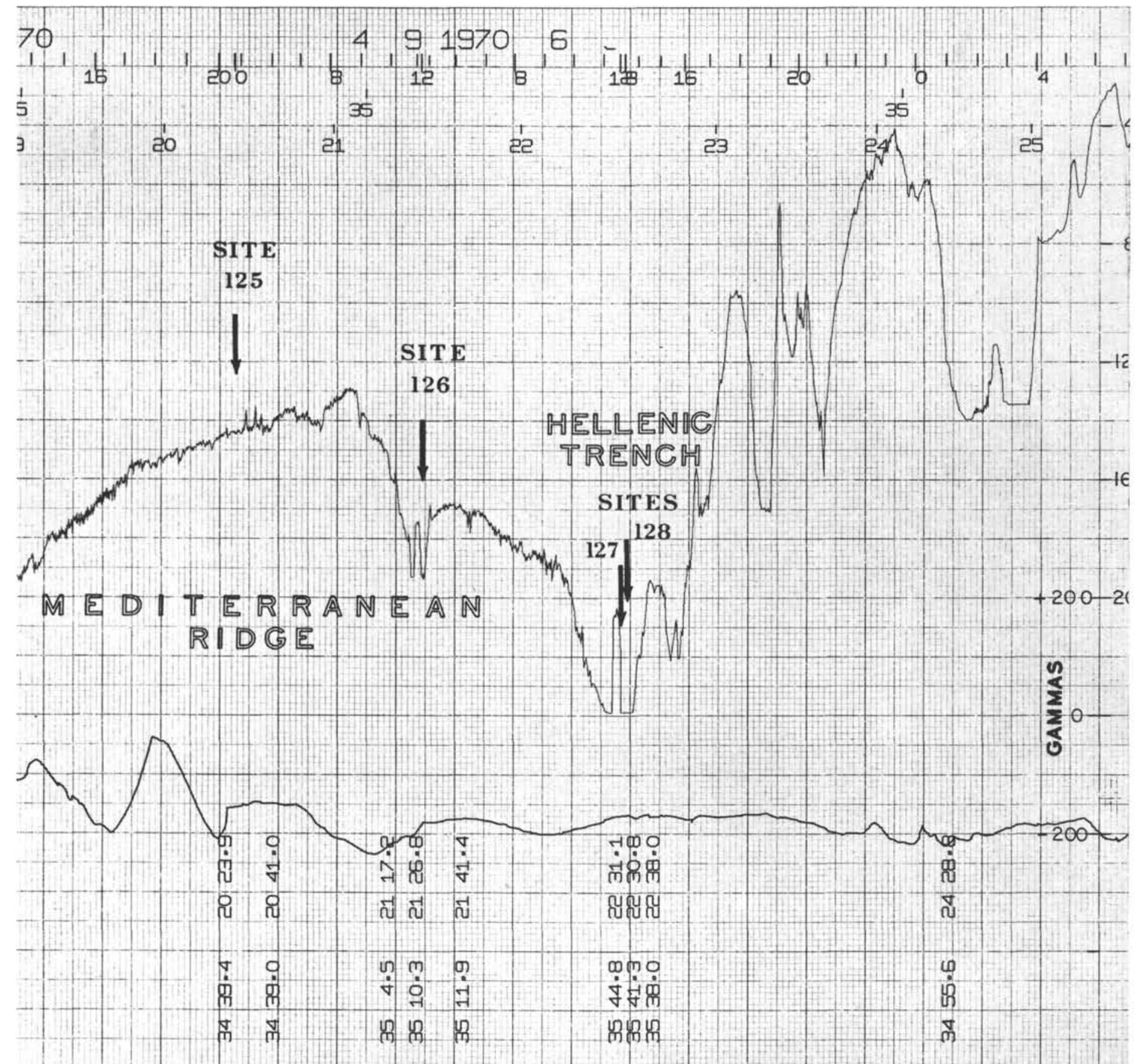
凹

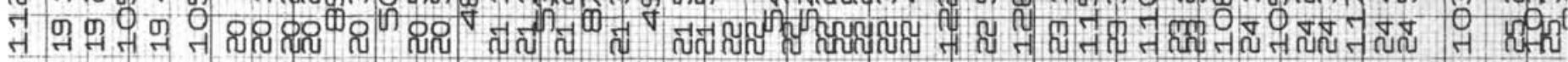

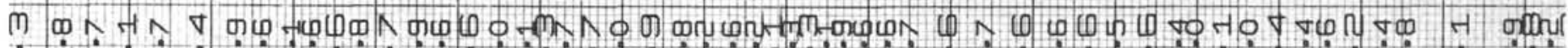
-

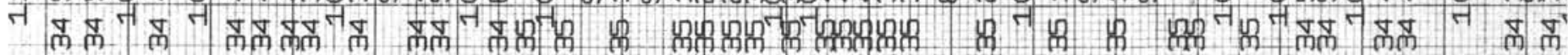

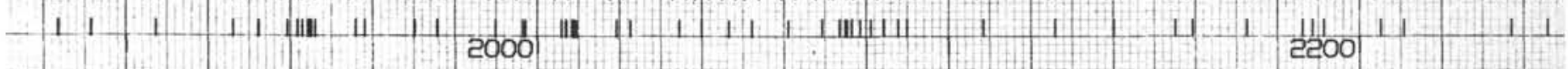

Figure 2F. 


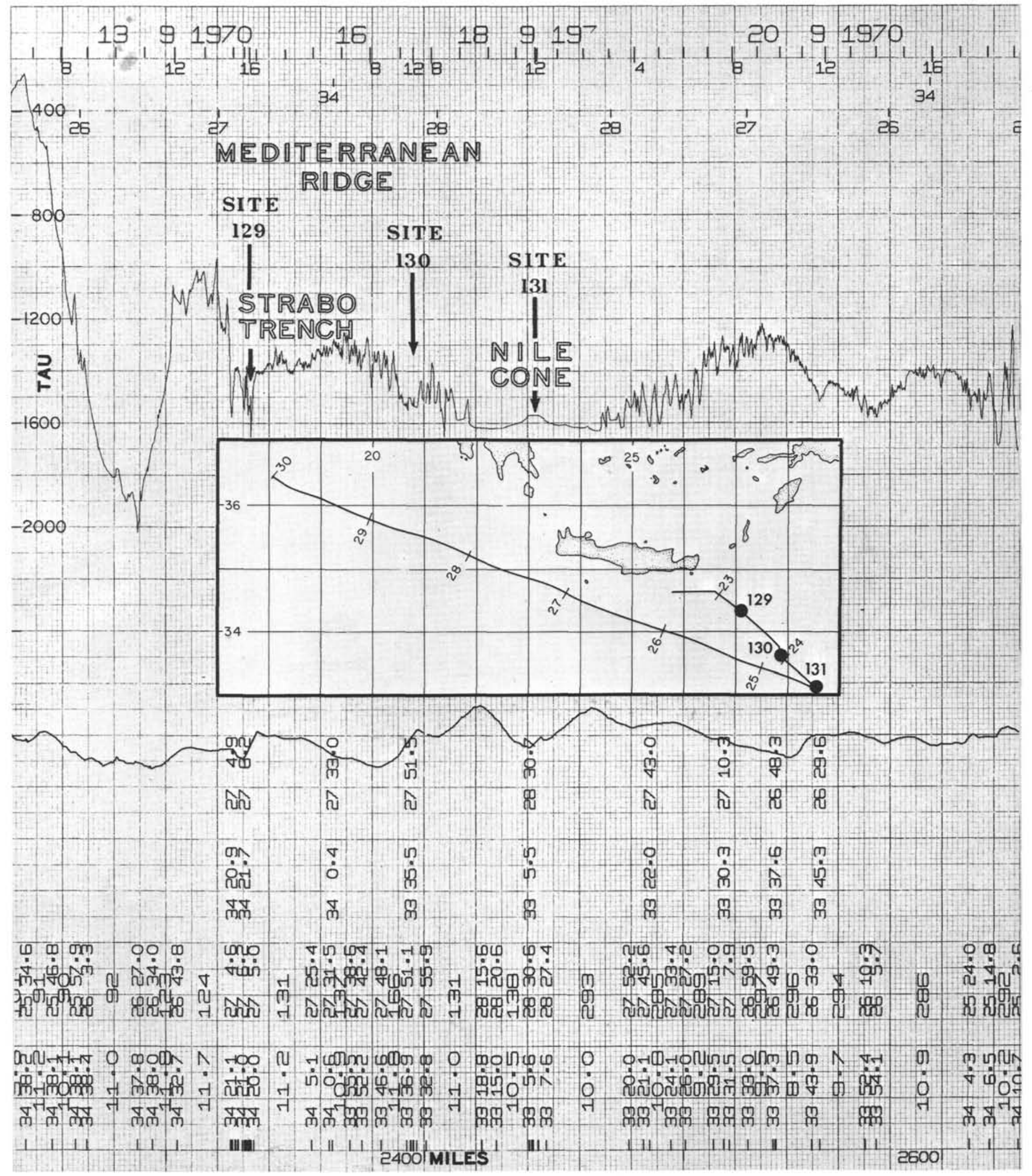

Figure 2G. 


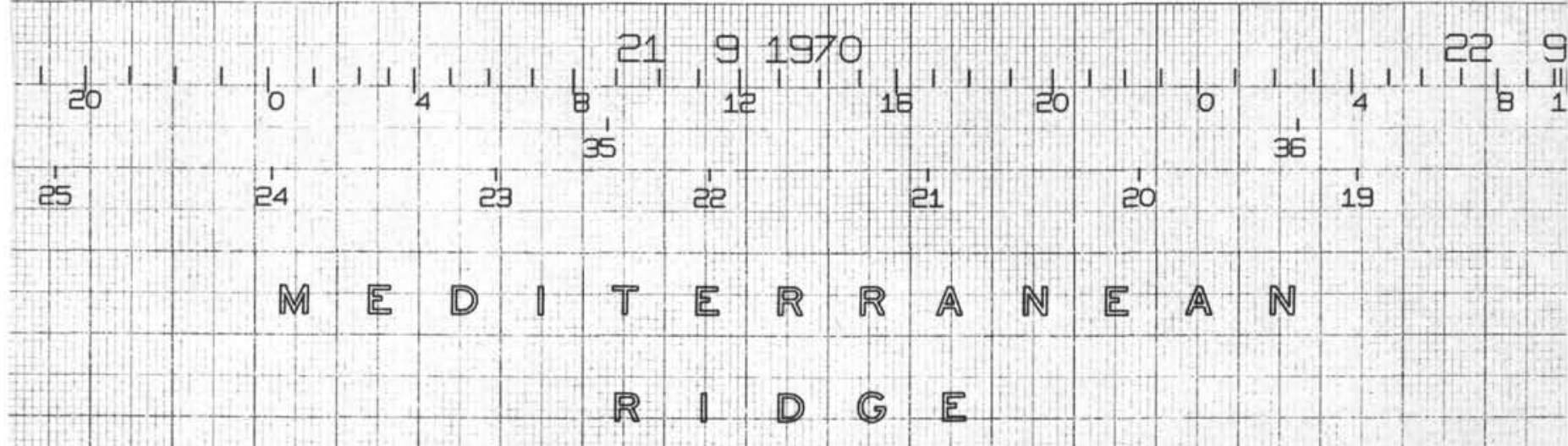
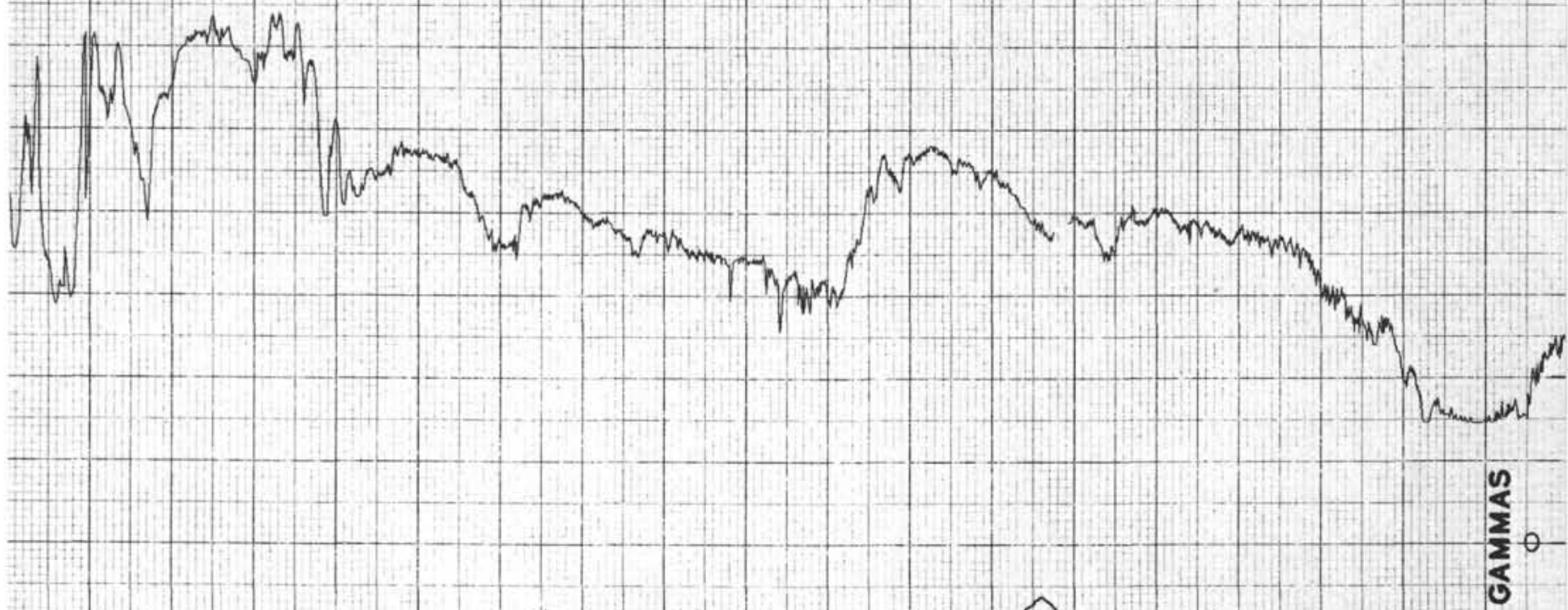

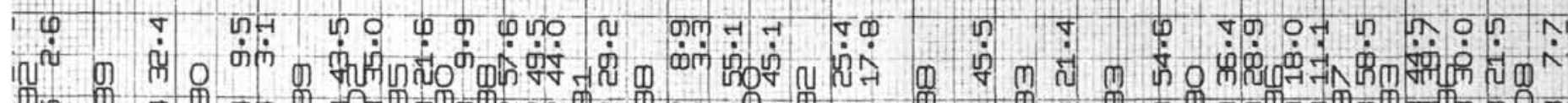

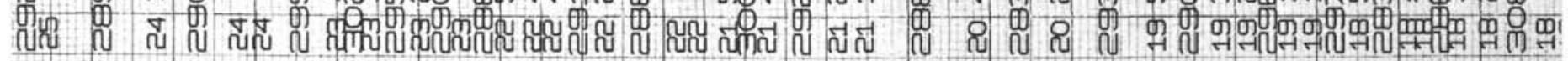

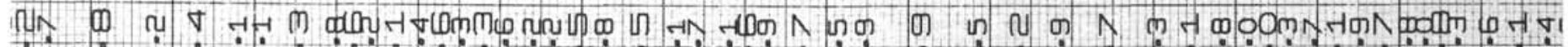

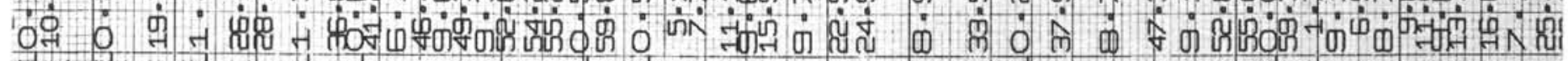
活

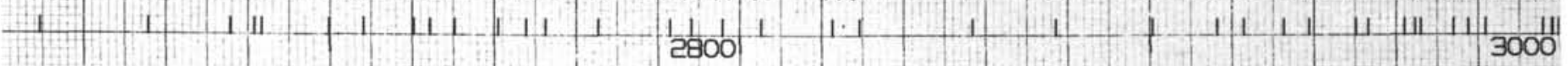

Figure $2 \mathrm{H}$. 


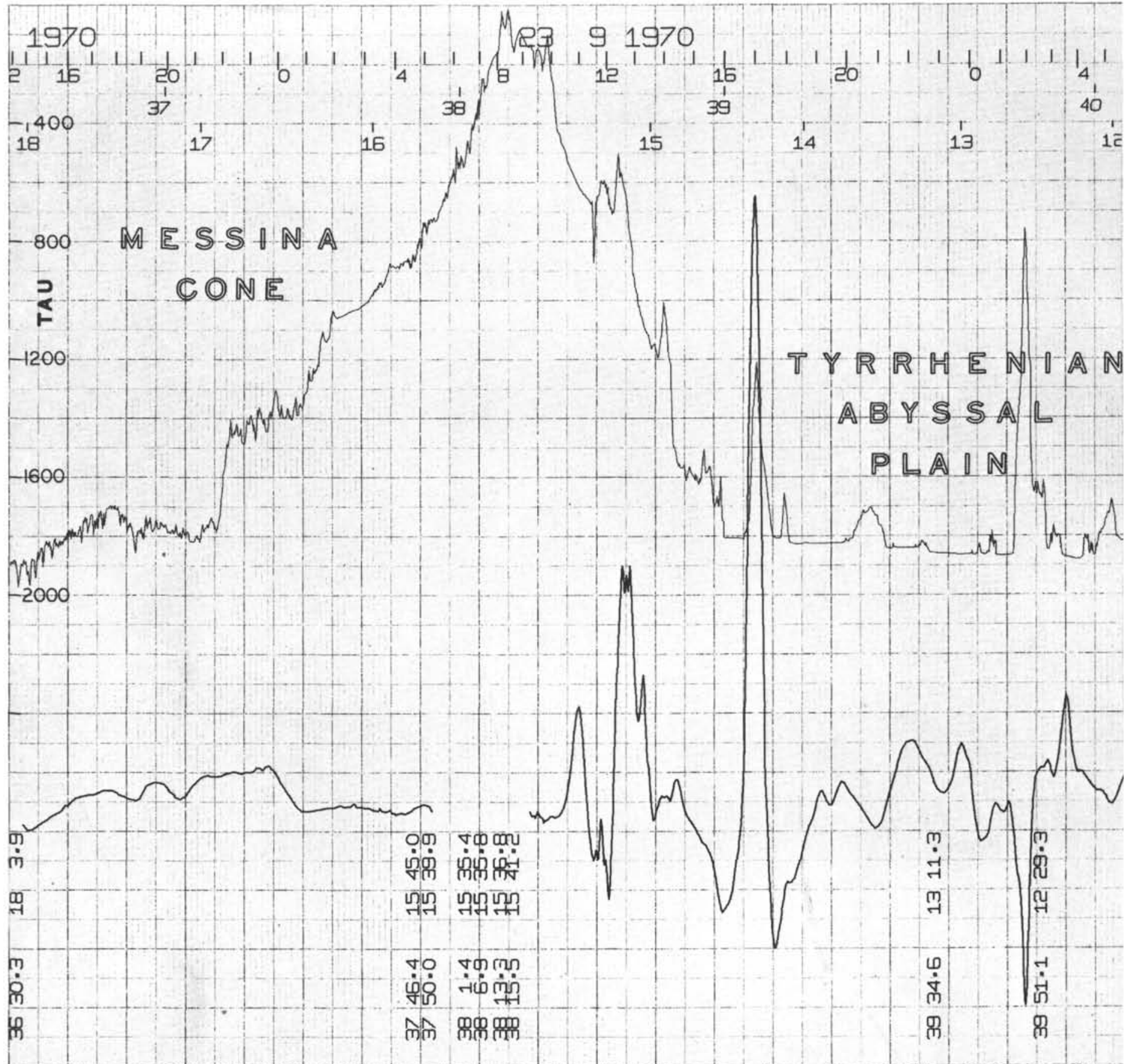

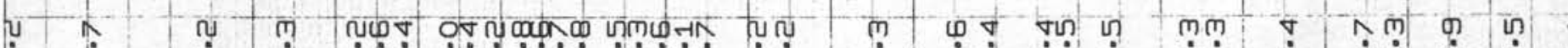

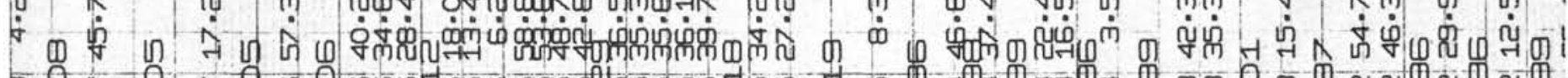
ए

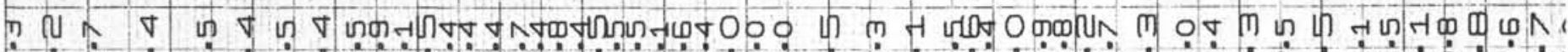

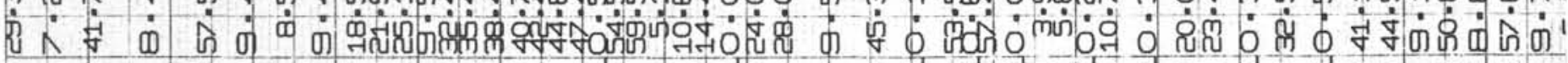

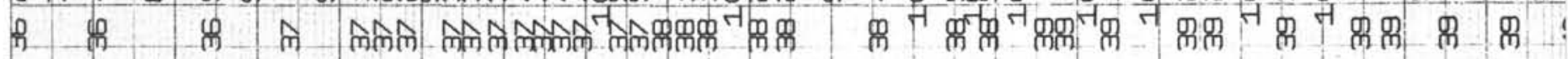

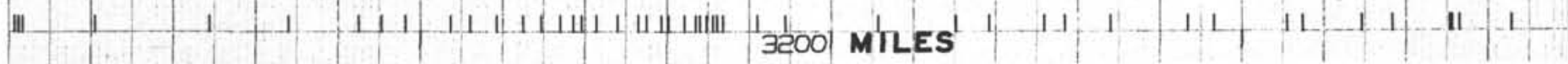

Figure 2I. 
24 2G, 9,1970
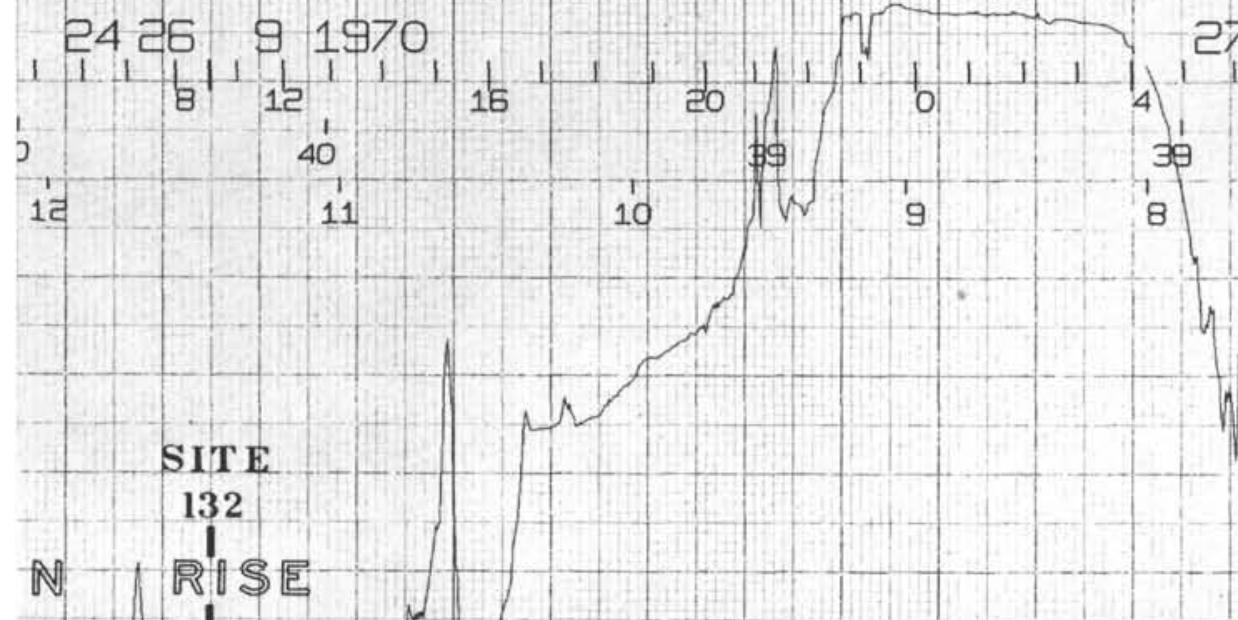

M SARDUNUA

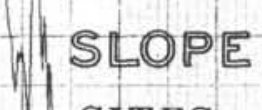

\section{SITES}

133
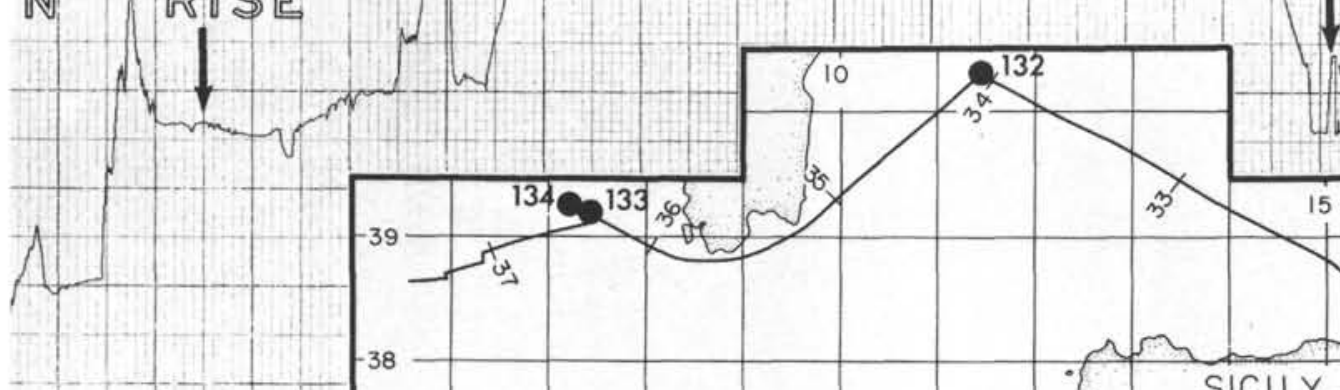

134

M

$$
\begin{aligned}
& \text { i் } \\
& \text { नु ने } \\
& \infty \quad \nabla \\
& \text { ที่ v } \\
& \text { ㅇ }
\end{aligned}
$$

\section{$\ln \sqrt{4} n_{\sqrt{n}}$}

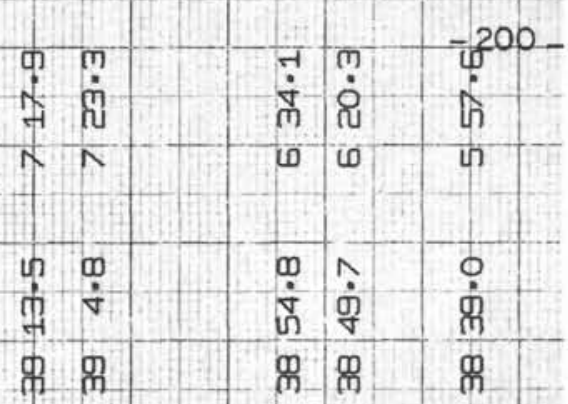

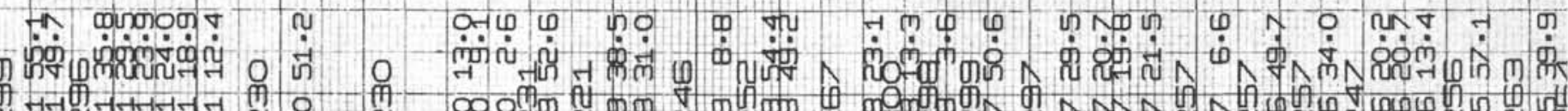

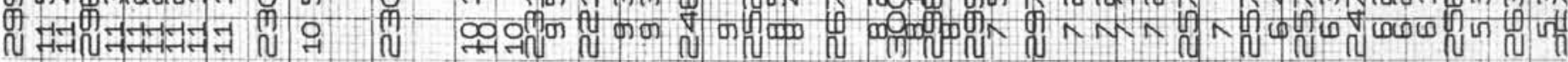

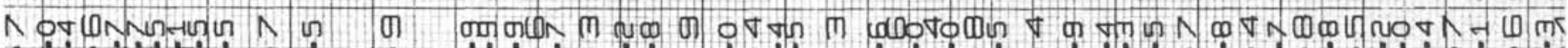

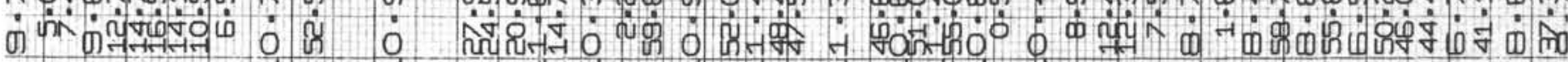

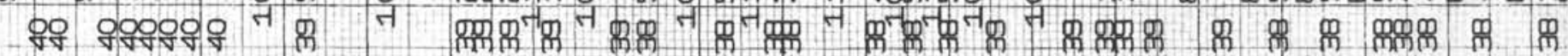

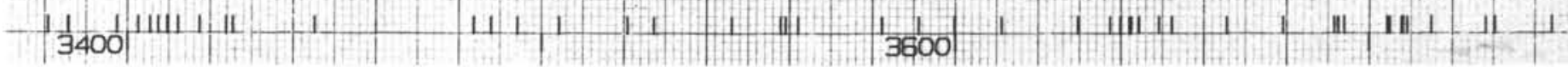

Figure 2J. 


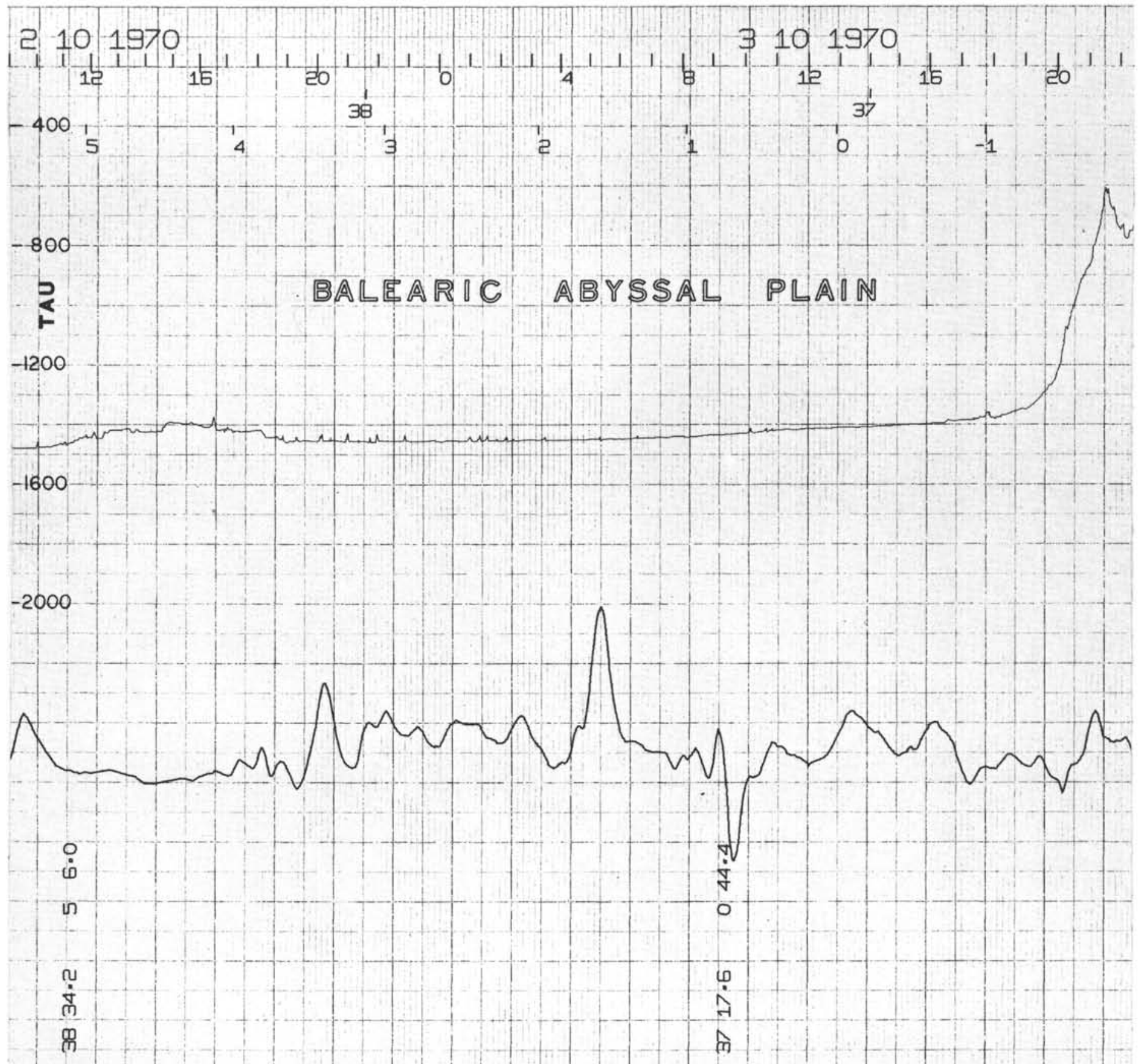

宽 $\begin{aligned} & m \\ & \dot{\omega}\end{aligned}$

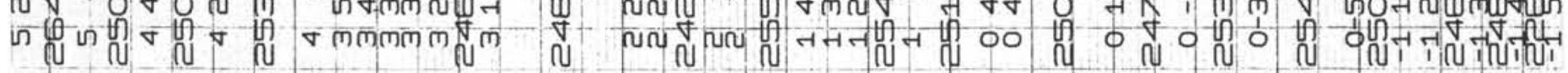

T

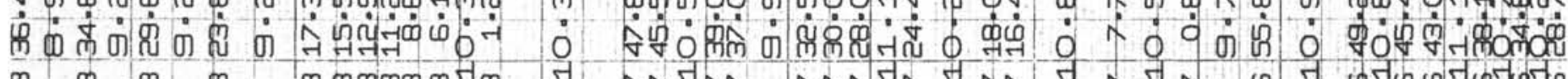

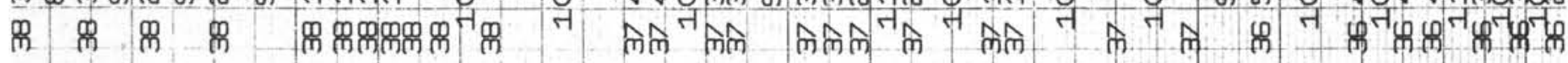

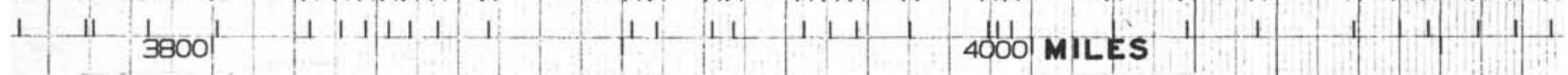

Figure 2K. 

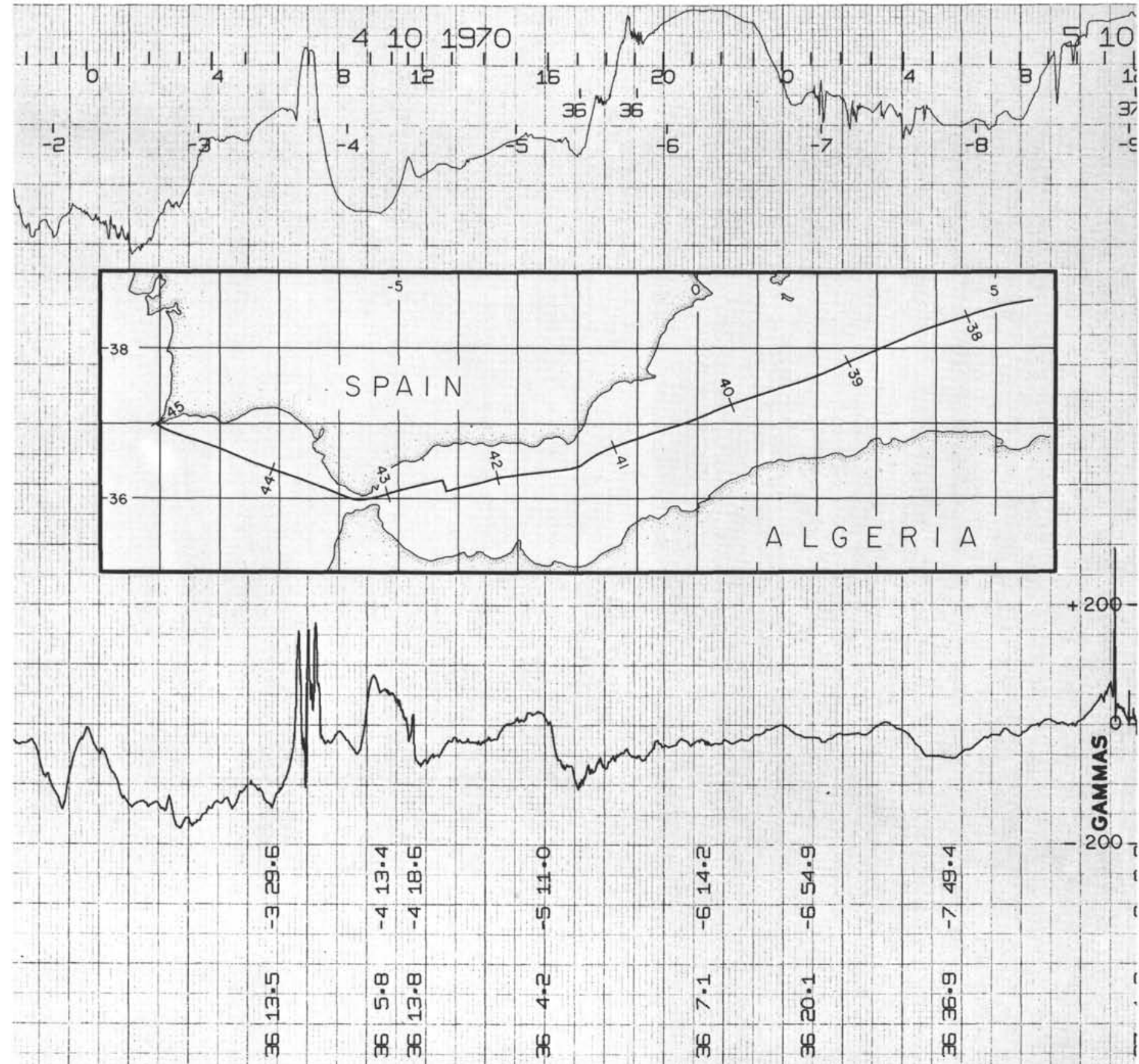

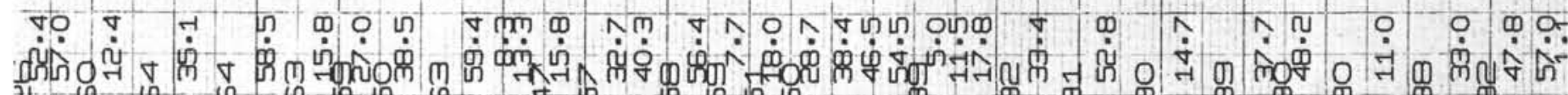
U⿳亠口冋丁

युगए ए

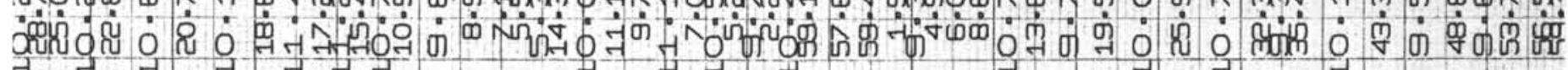

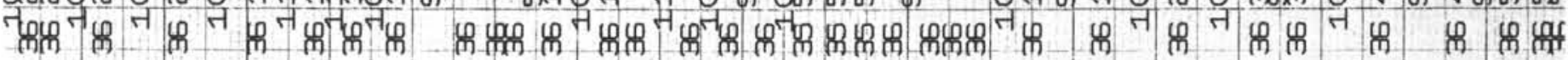

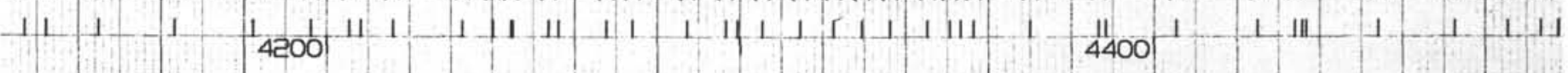

Figure 2L. 


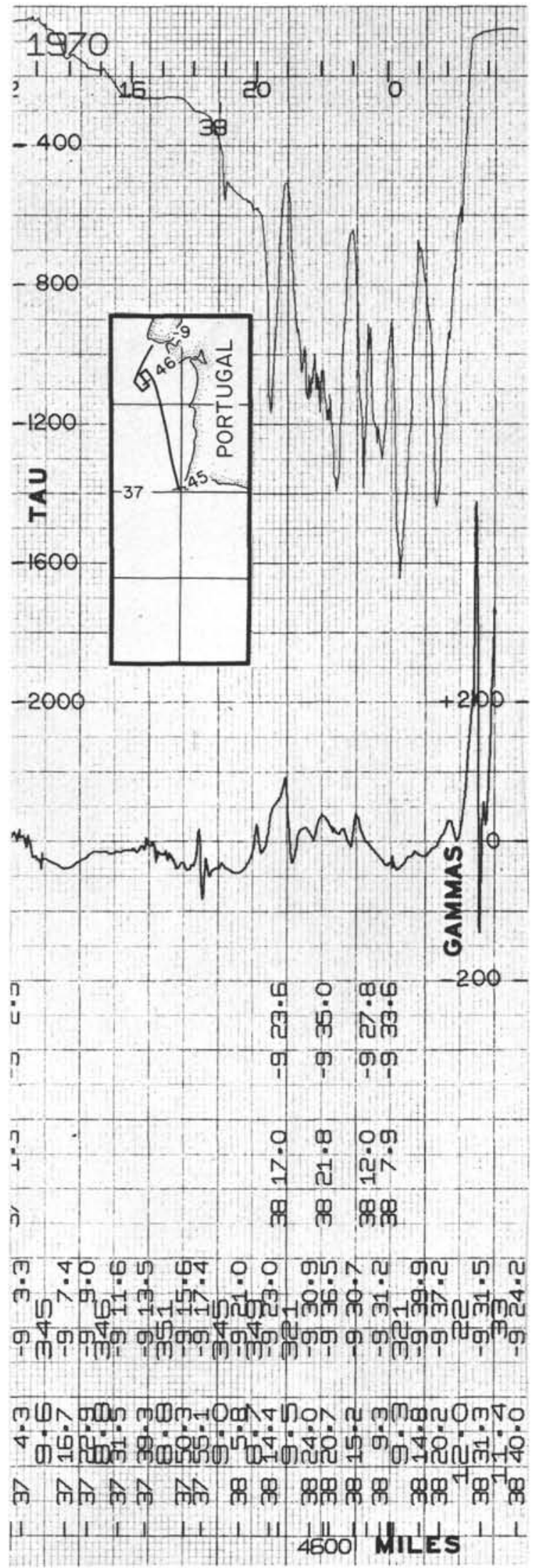

Figure 2M. 


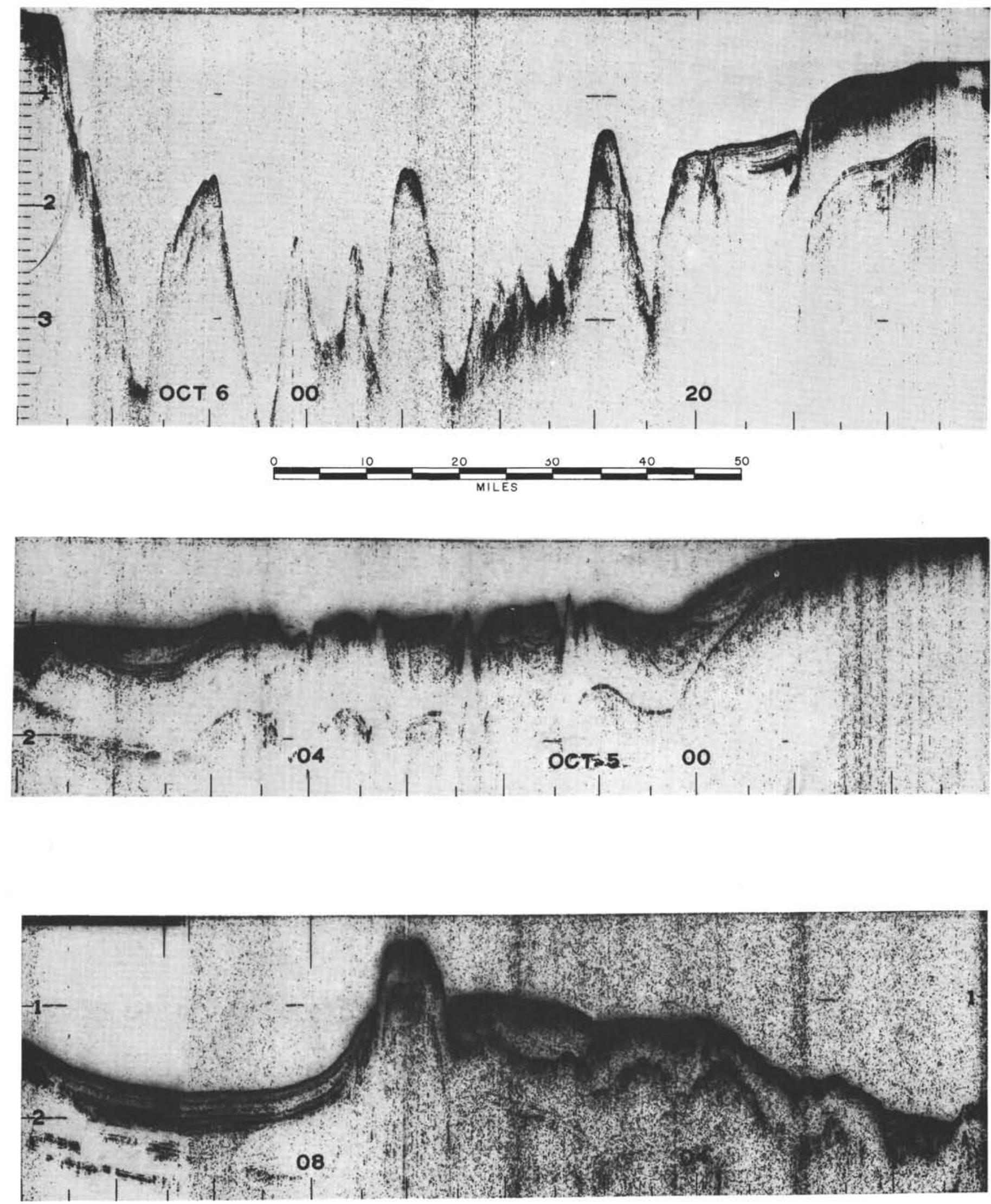

Figure 3A. Seismic reflection profiles. 

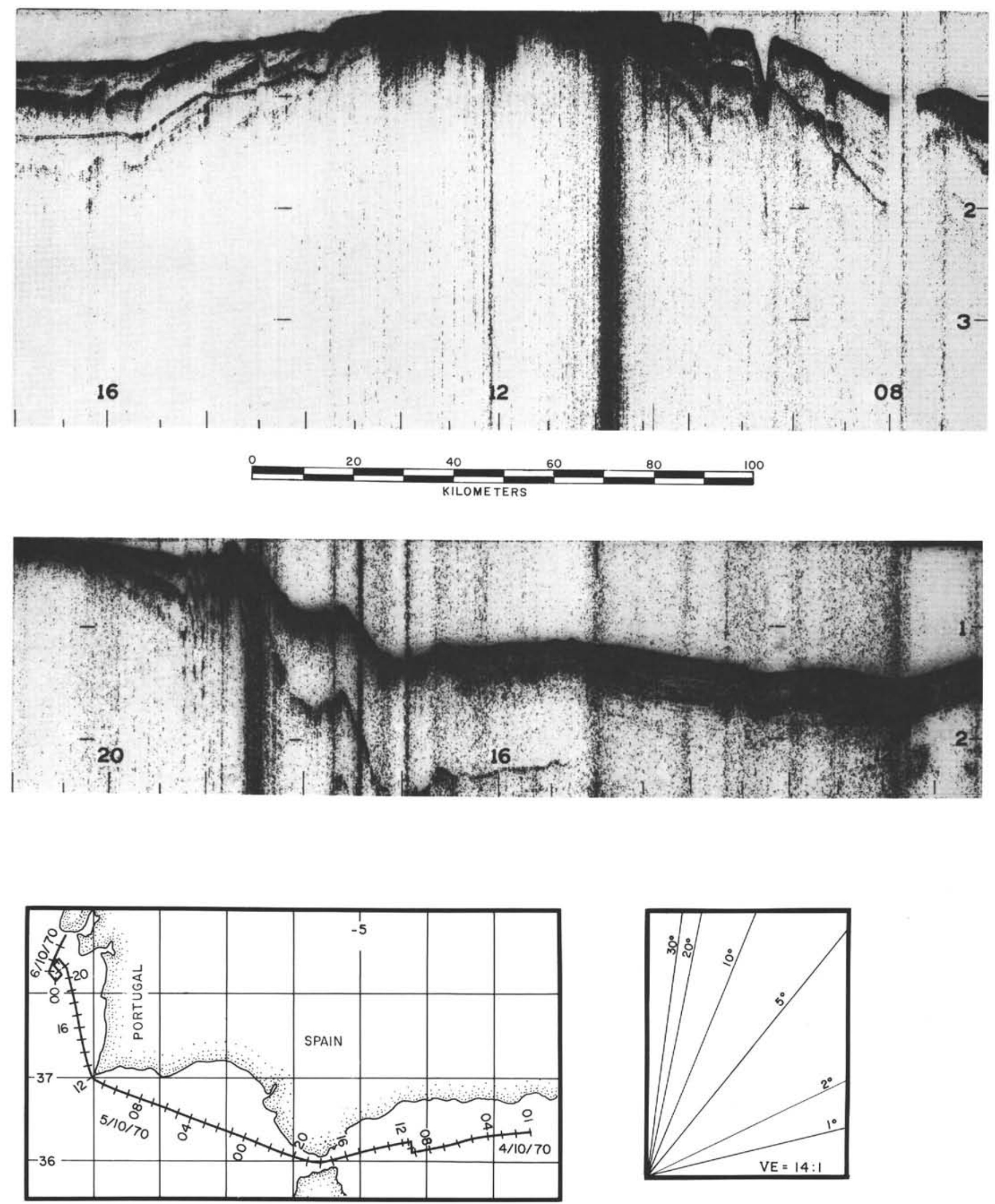

Figure 3B.

Vertical scale in seconds of two-way travel time. 

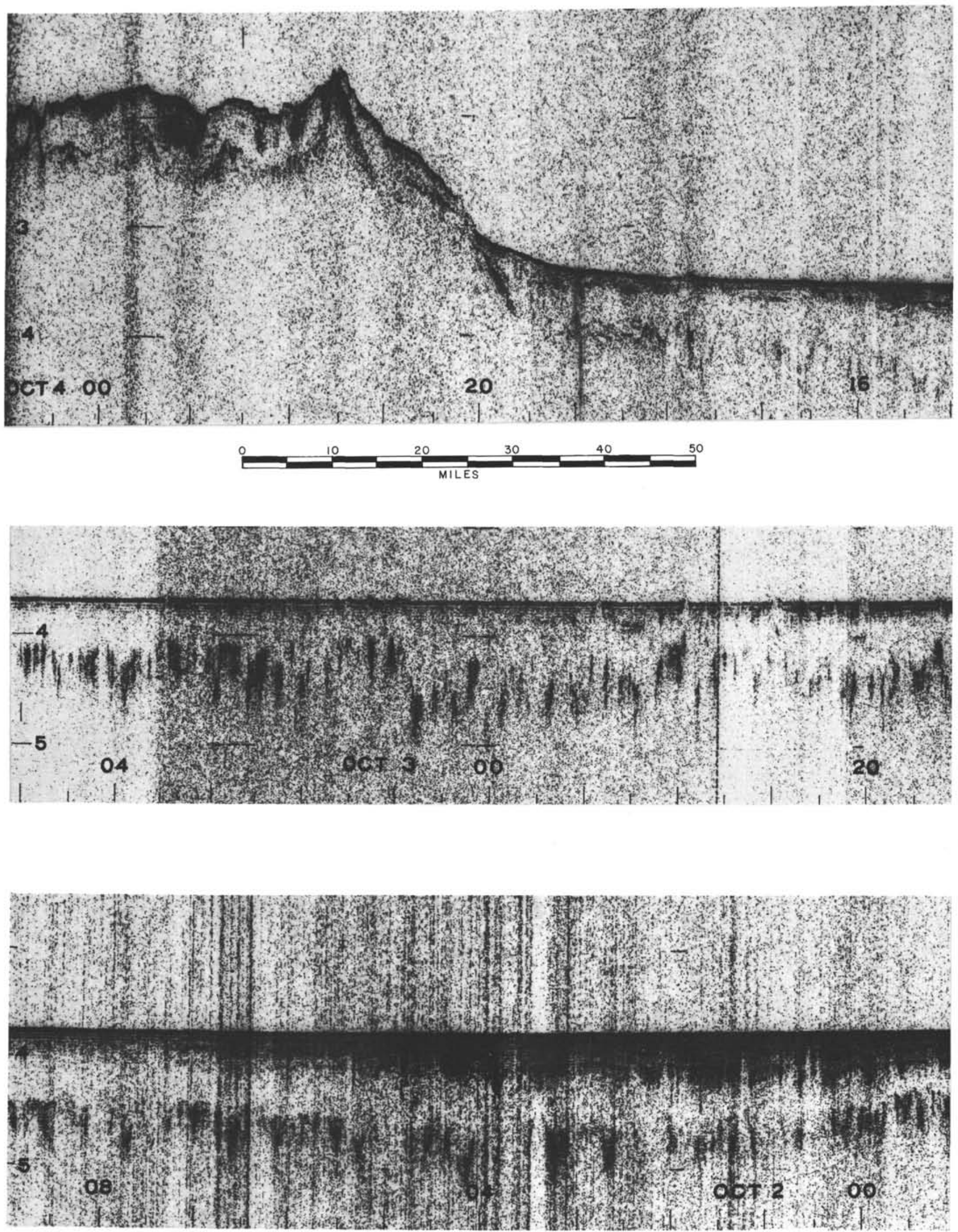

Figure 3C. 

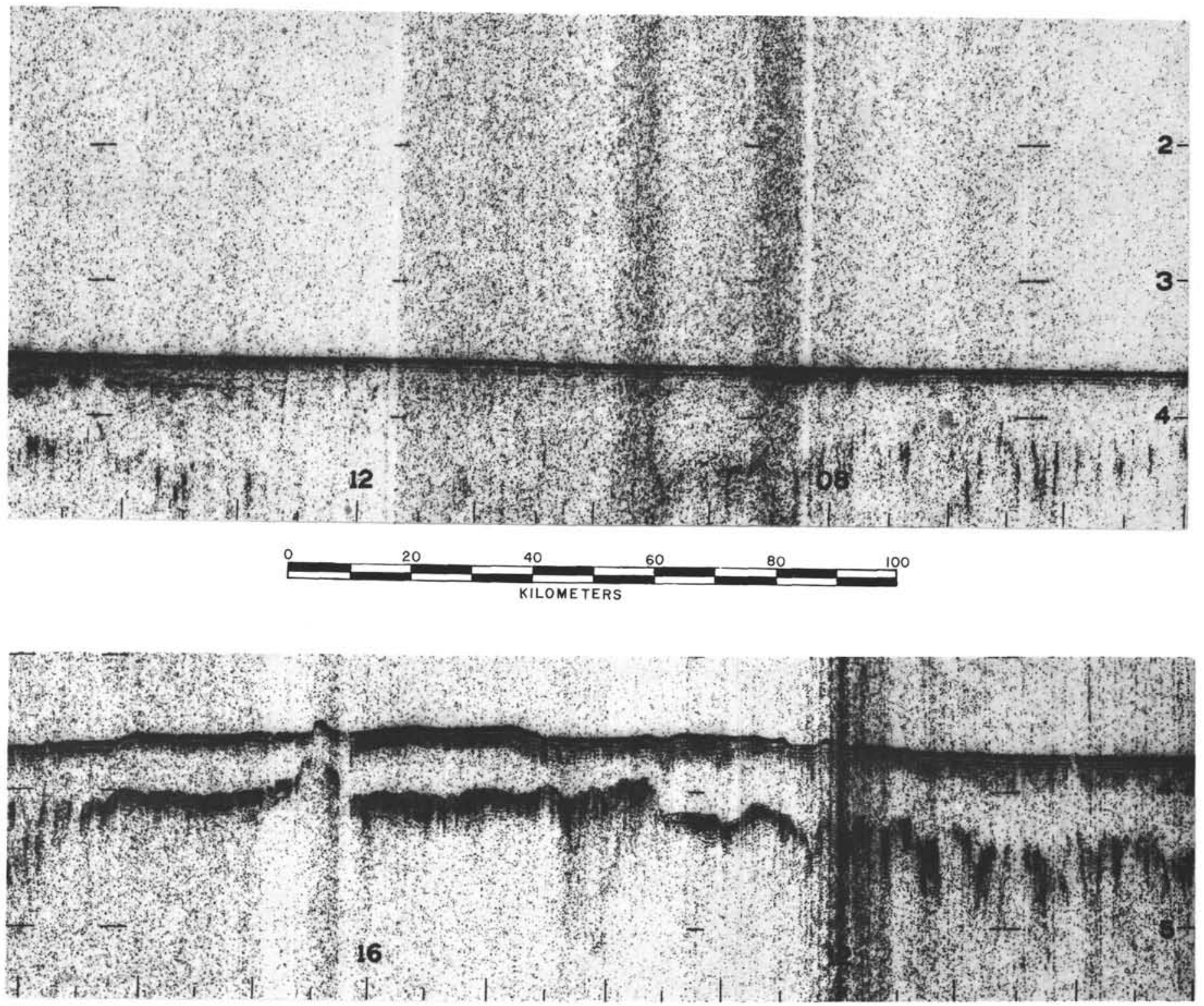

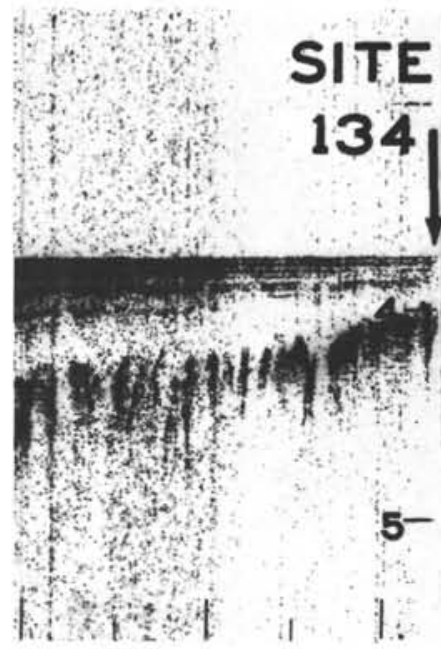

Figure 3D.

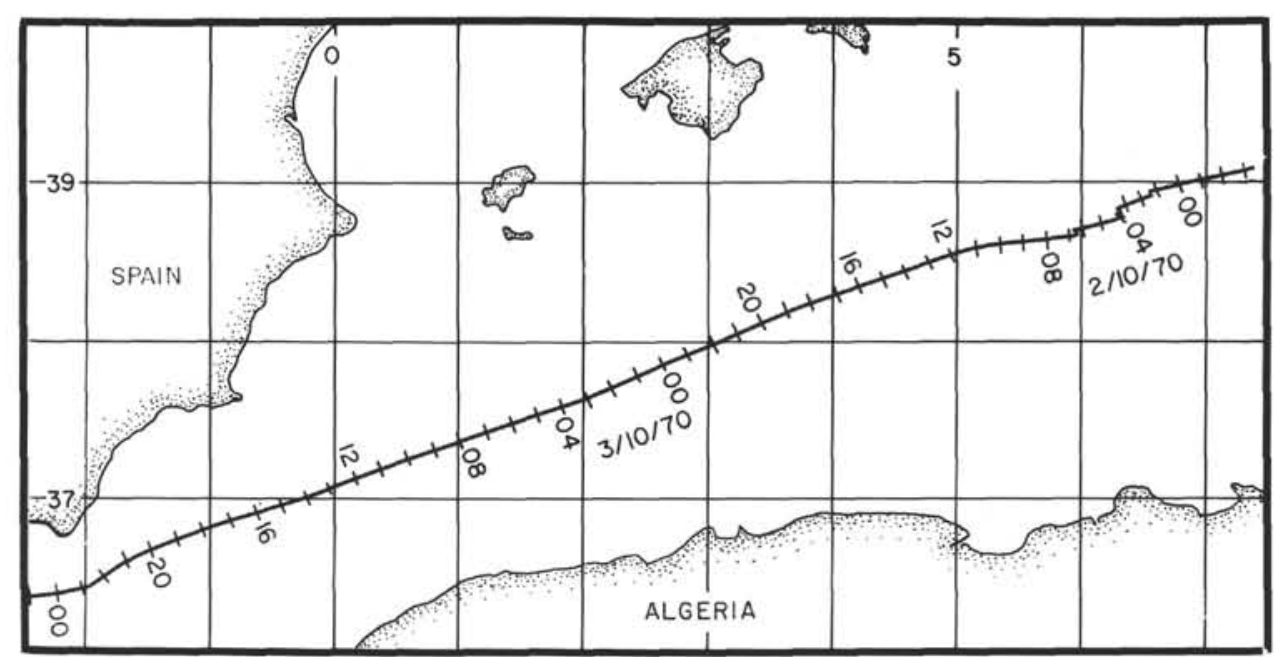

Vertical scale in seconds of two-way travel time. 
W. B. F. RYAN, T. B. GUSTAFSON
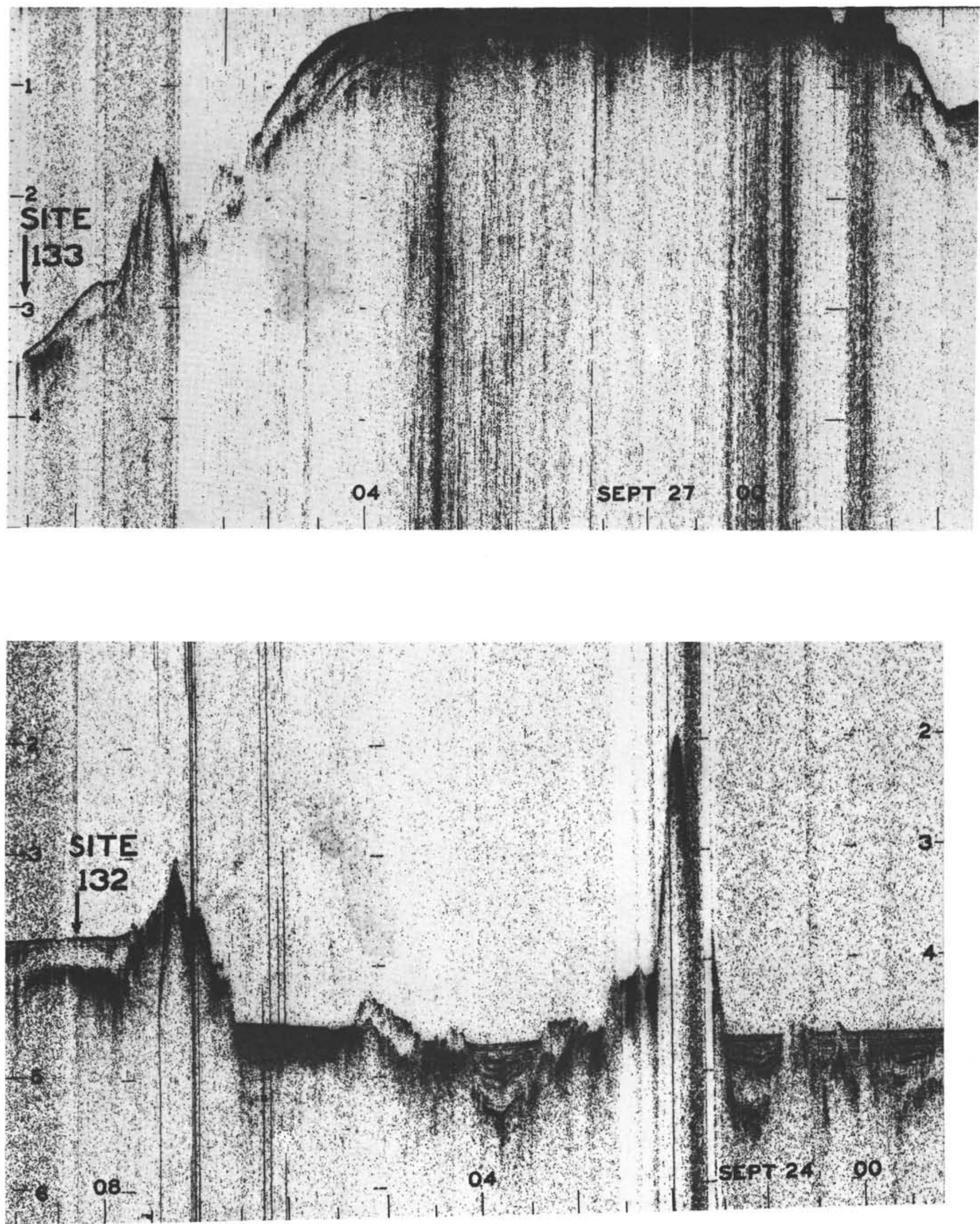

Figure $3 E$. 

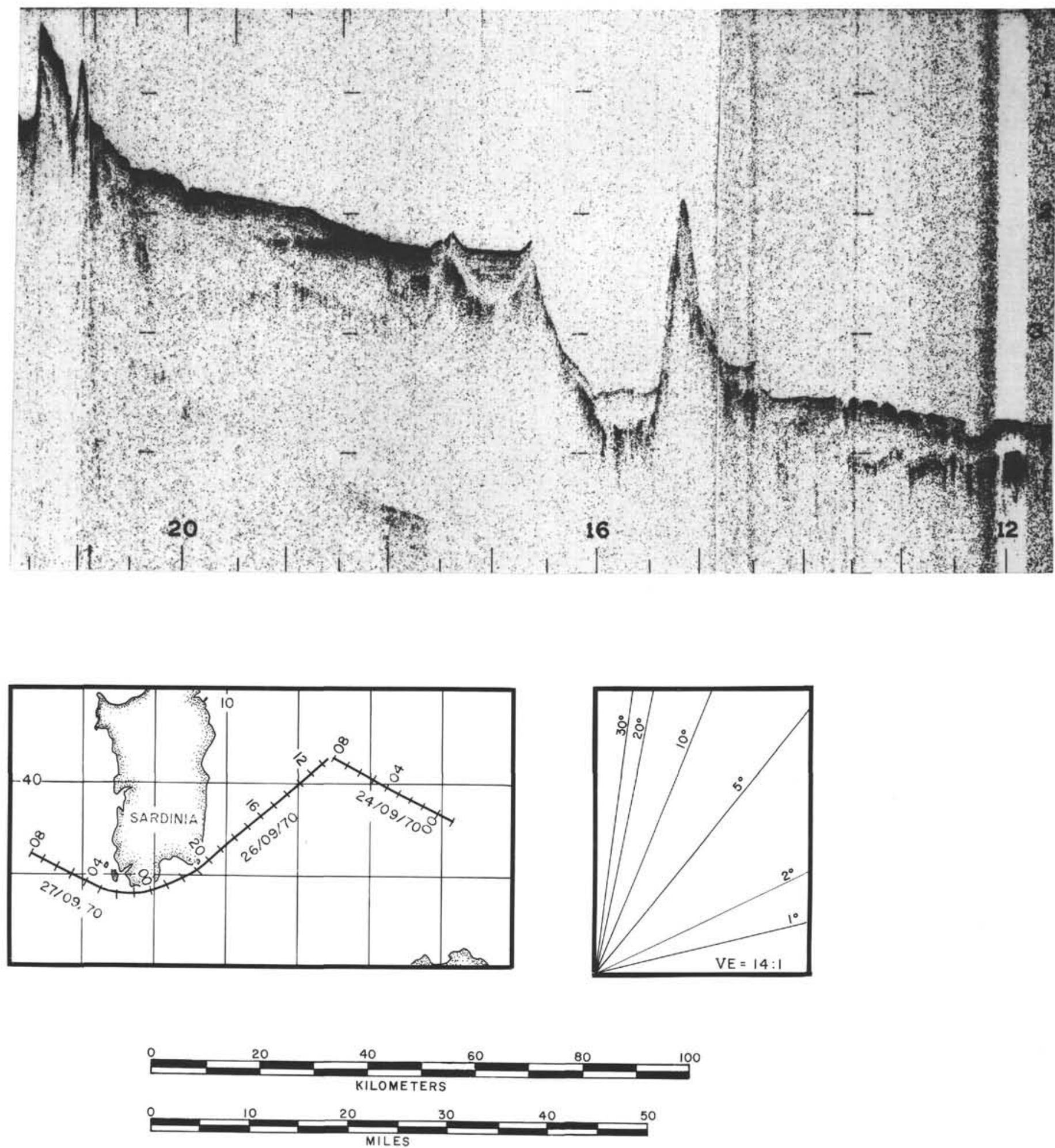

Figure $3 \mathrm{~F}$.

Vertical scale in seconds of two-way travel time. 

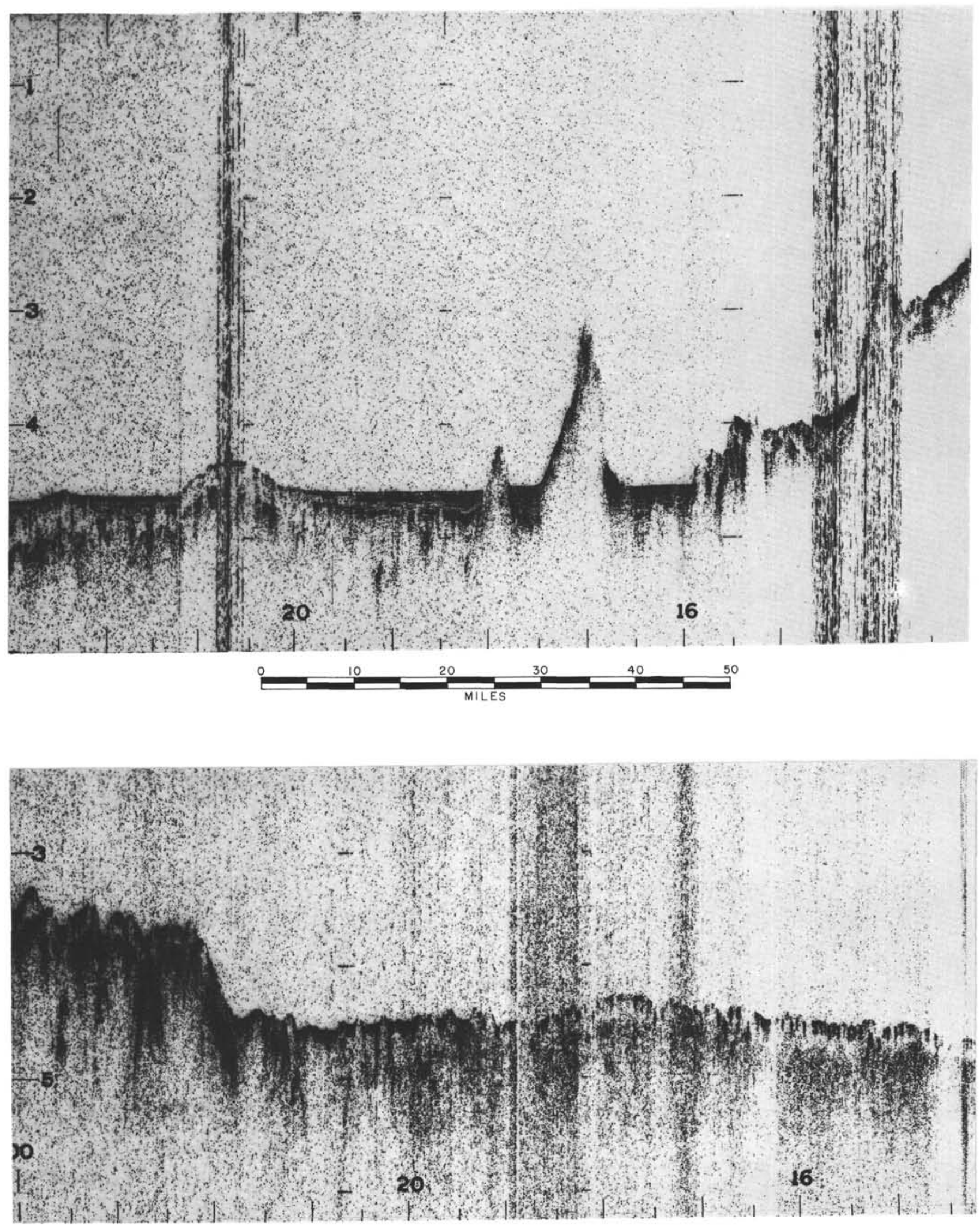

Figure 3G. 

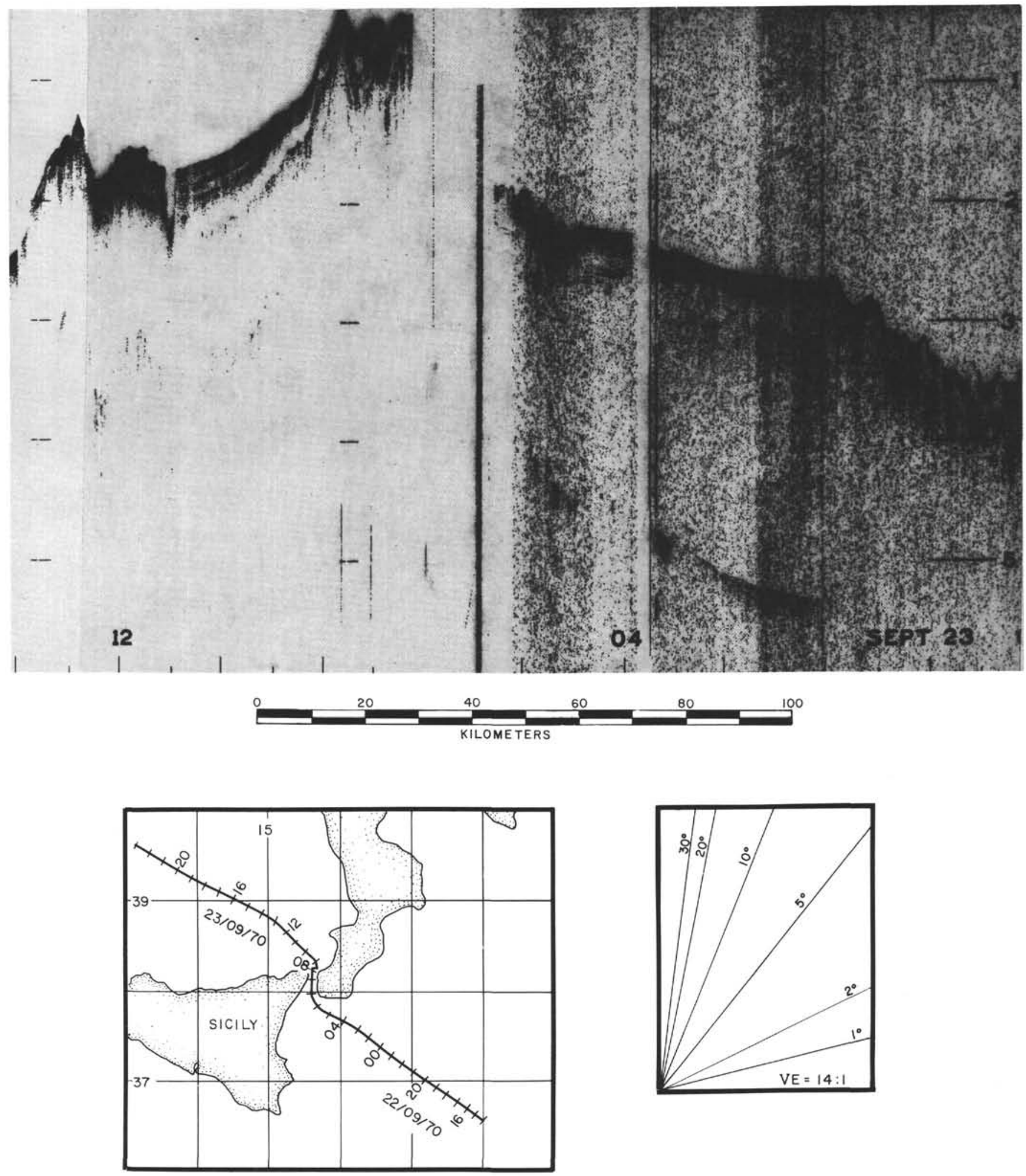

Figure $3 \mathrm{H}$.

Vertical scale in seconds of two-way travel time. 
W. B. F. RYAN, T. B. GUSTAFSON
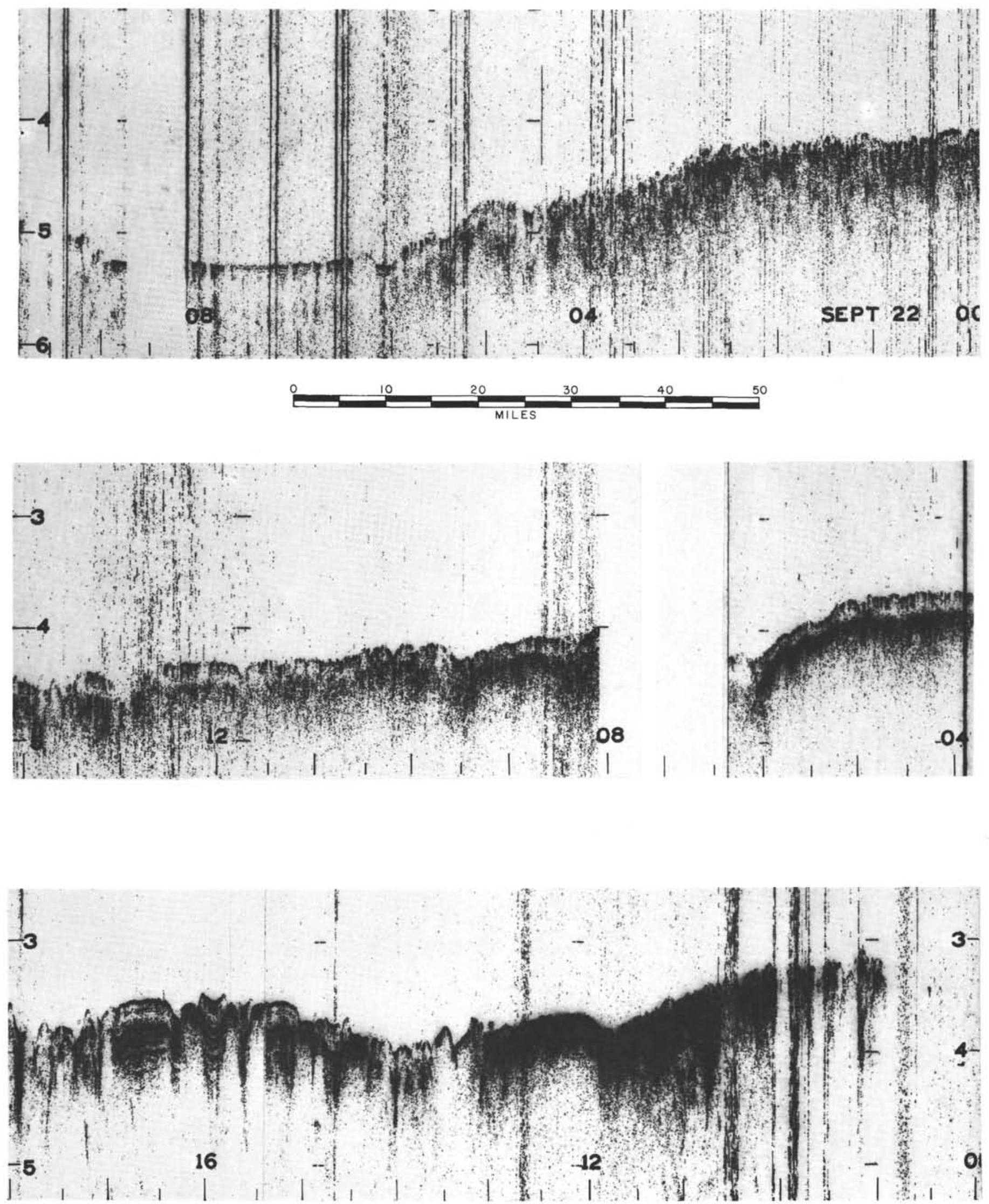

Figure 3I. 

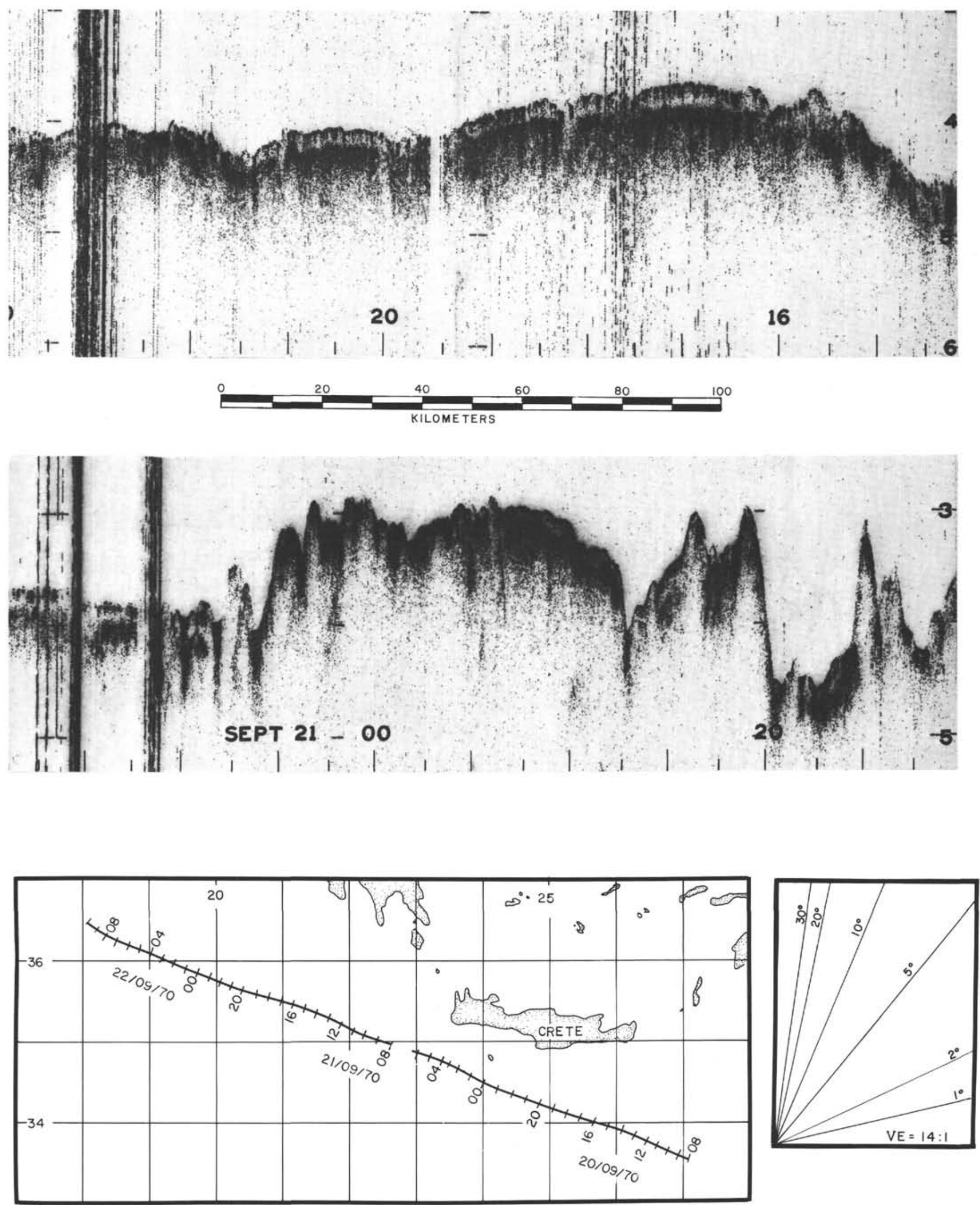

Figure 3J.

Vertical scale in seconds of two-way travel time. 

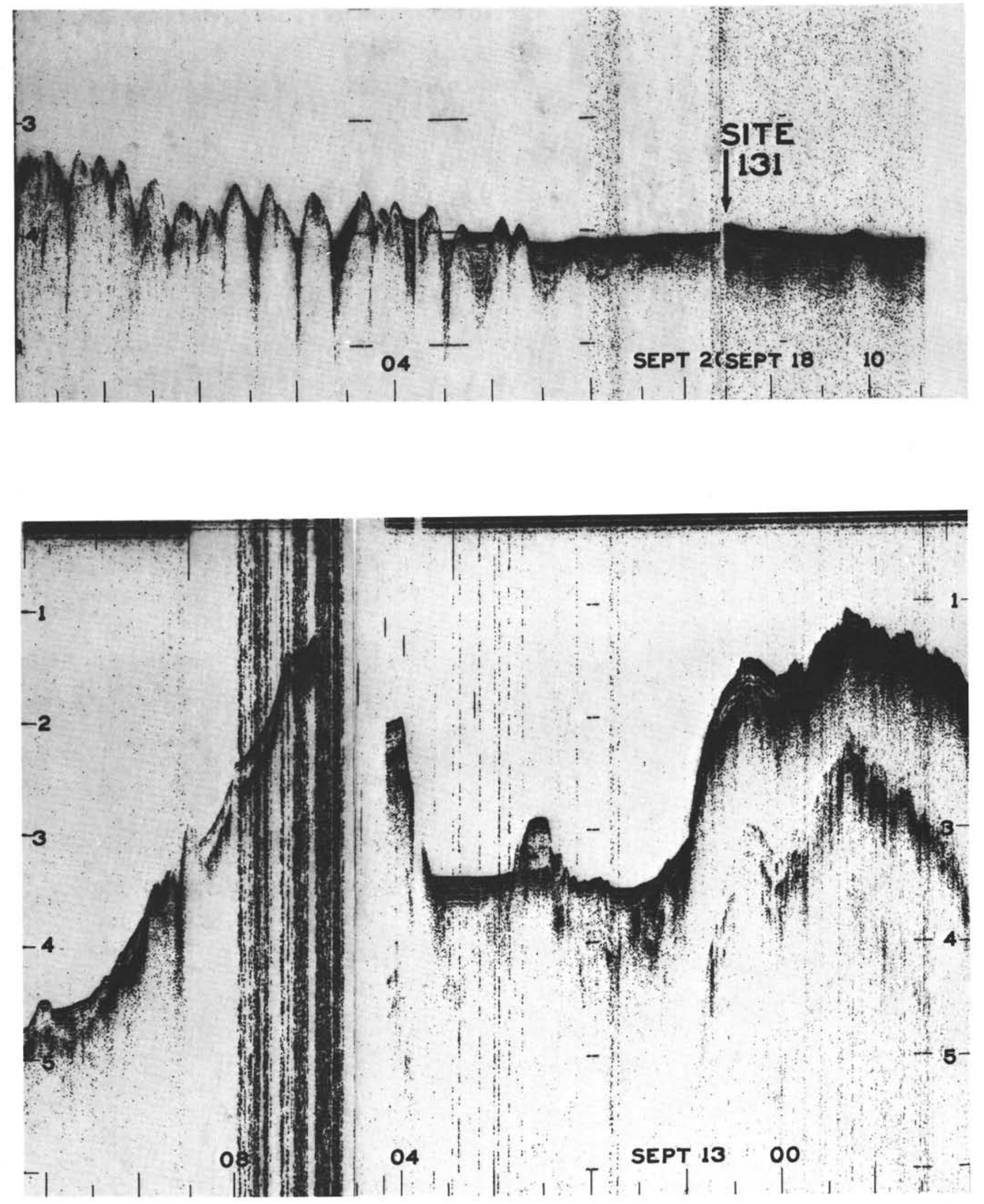

Figure $3 \mathrm{~K}$. 

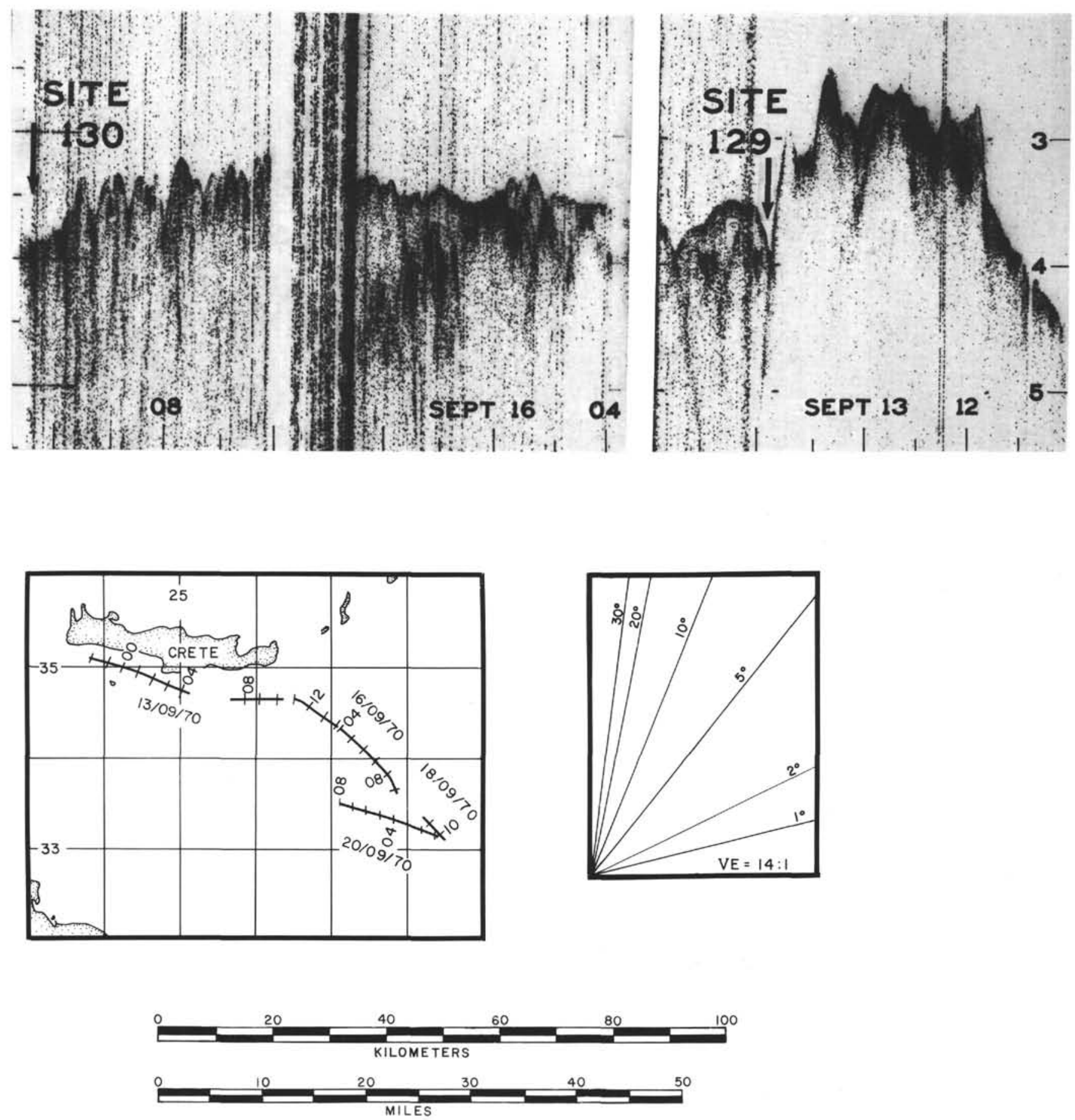

Figure 3L. Vertical scale in seconds of two-way travel time. 

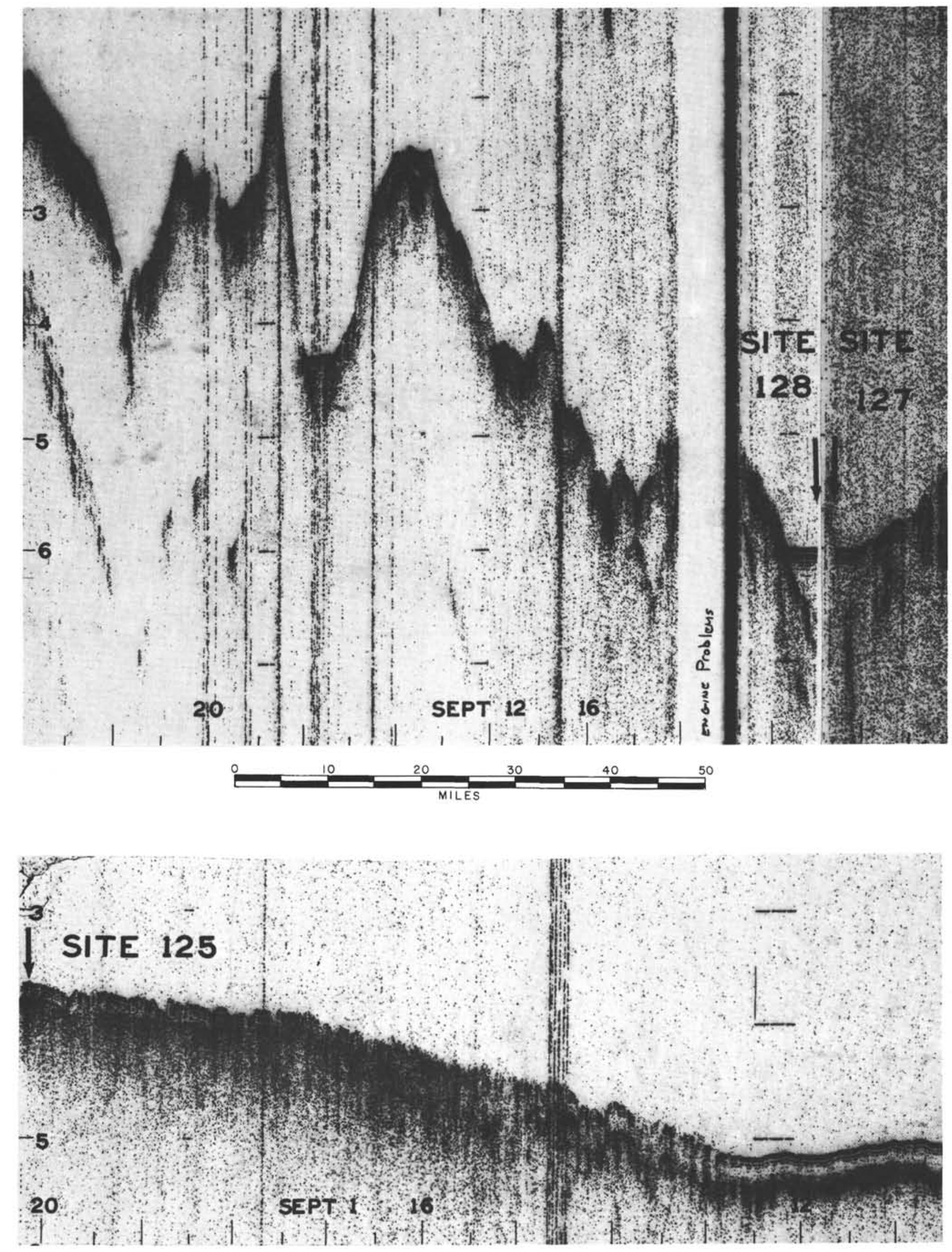

Figure 3M. 

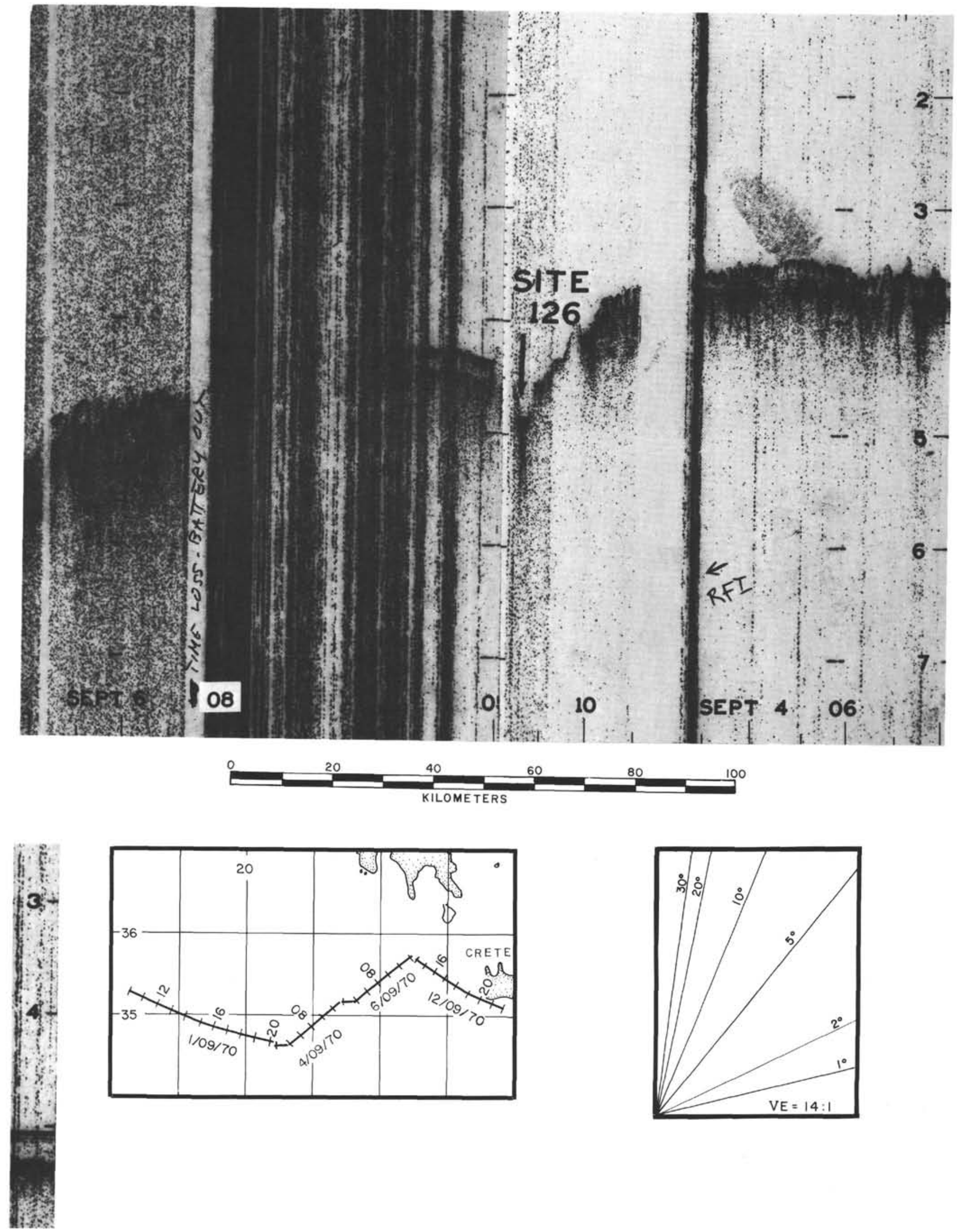

Figure 3N.

Vertical scale in seconds of two-way travel time. 

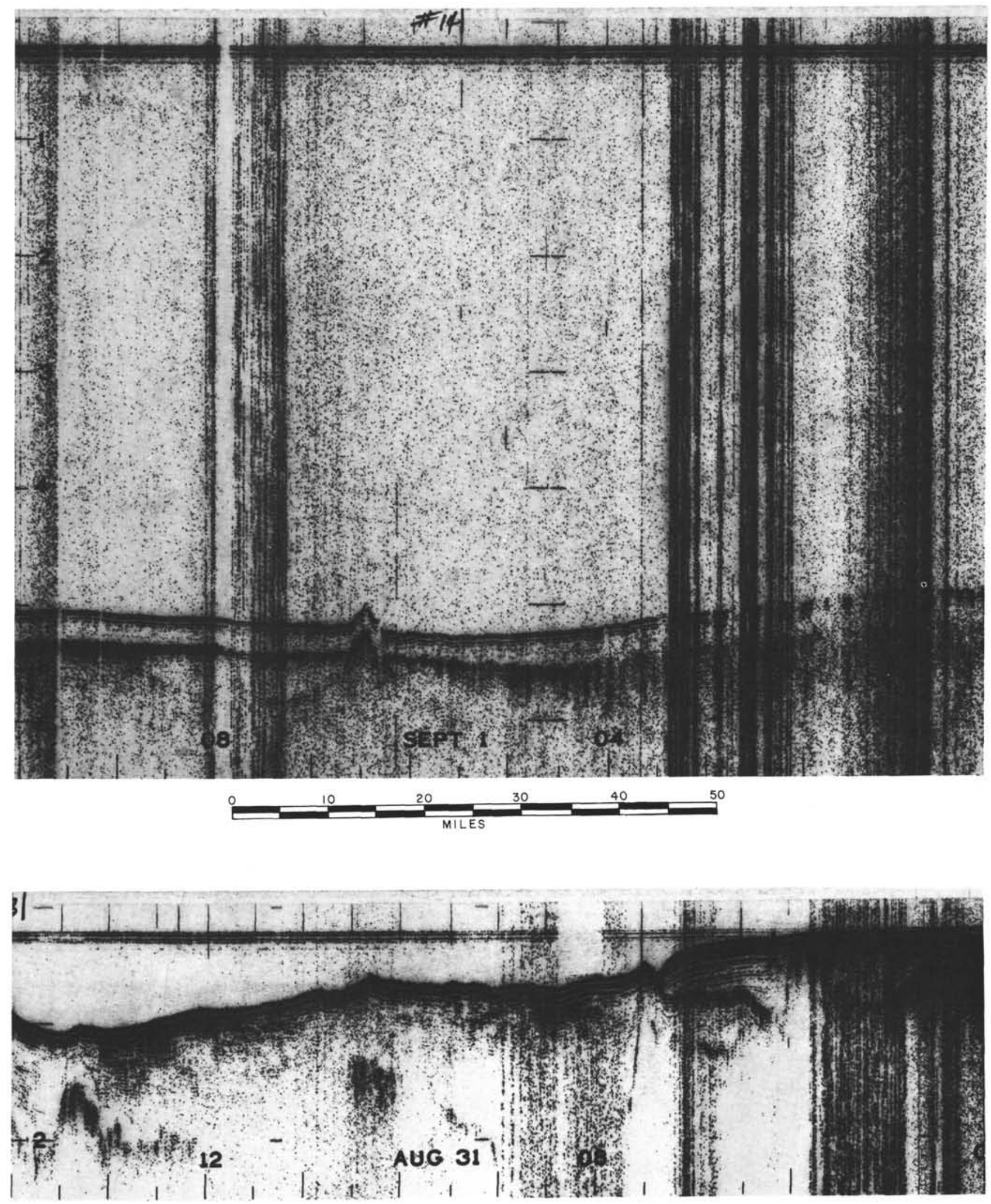

Figure 30. 

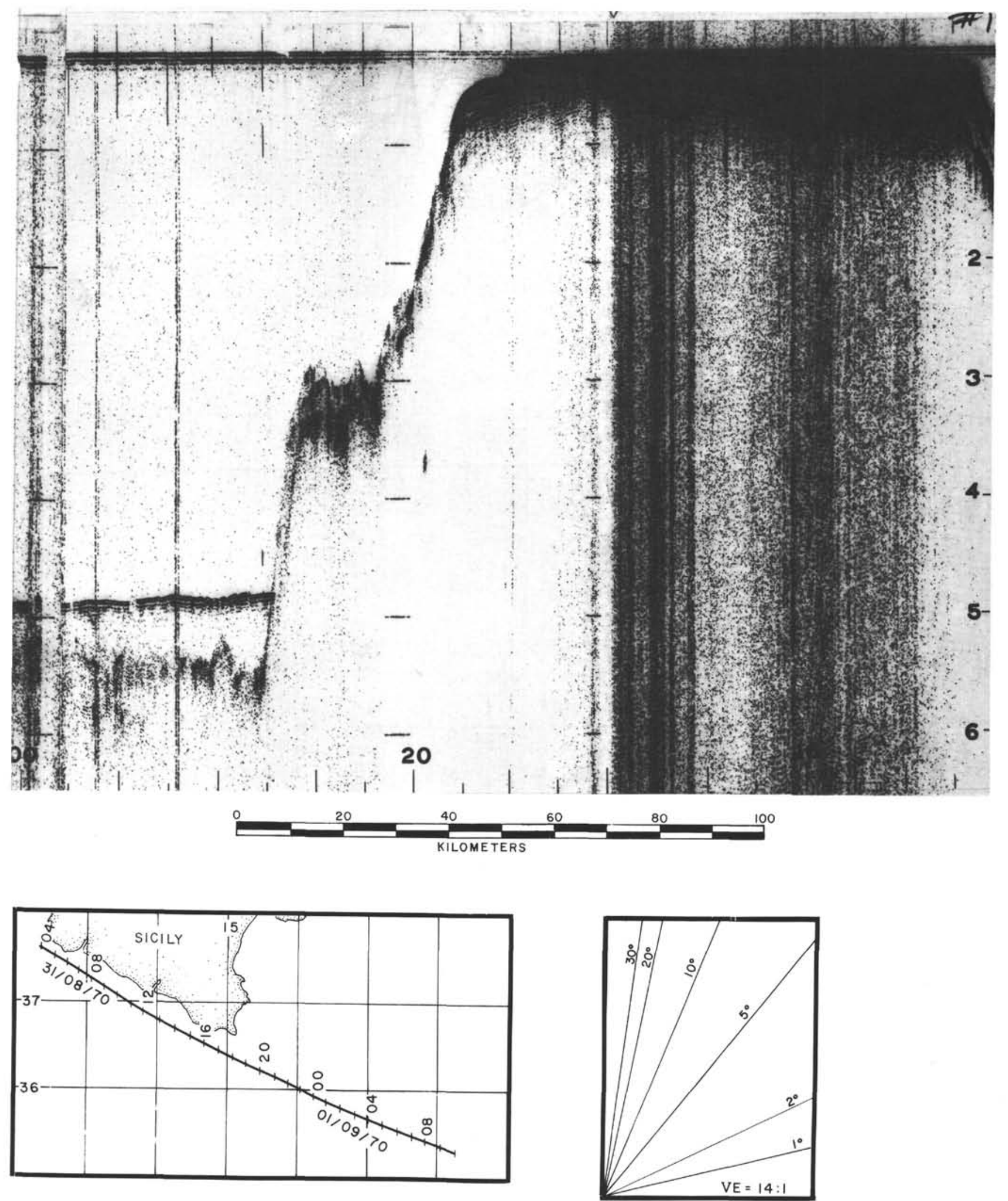

Figure 3P.

Vertical scale in seconds of two-way travel time. 

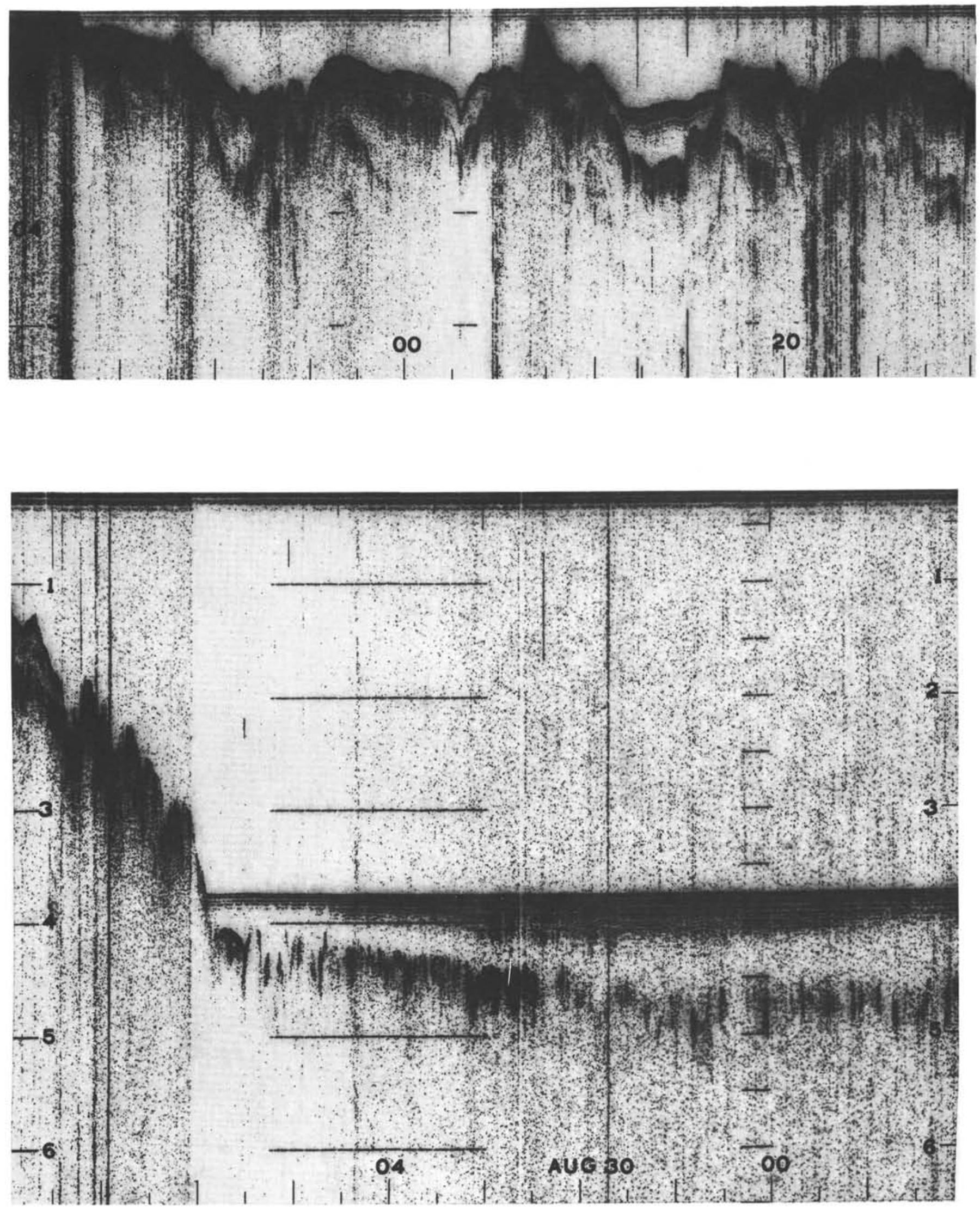

Figure $3 \mathrm{Q}$. 

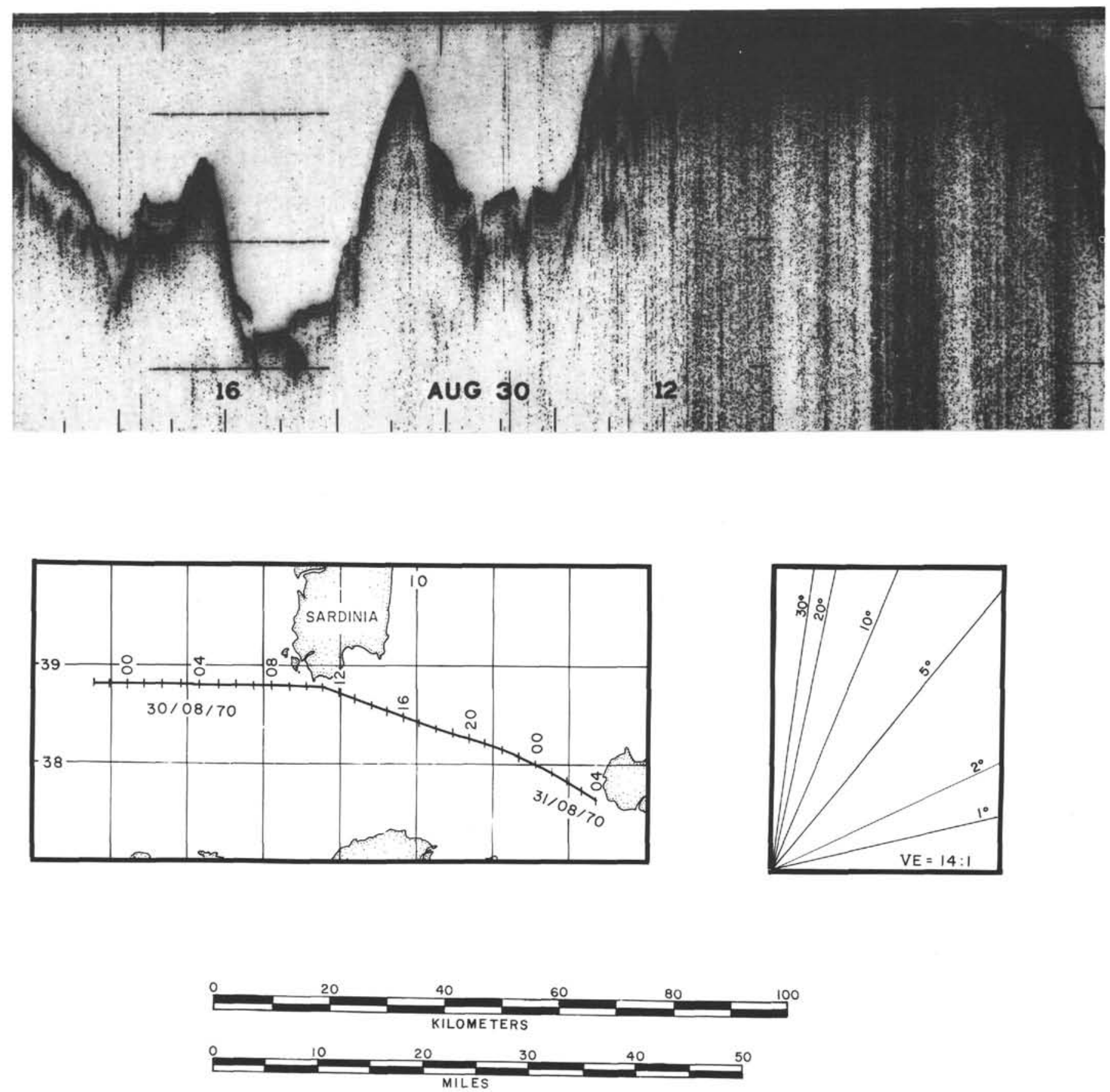

Figure 3R. Vertical scale in seconds of two-way travel time. 

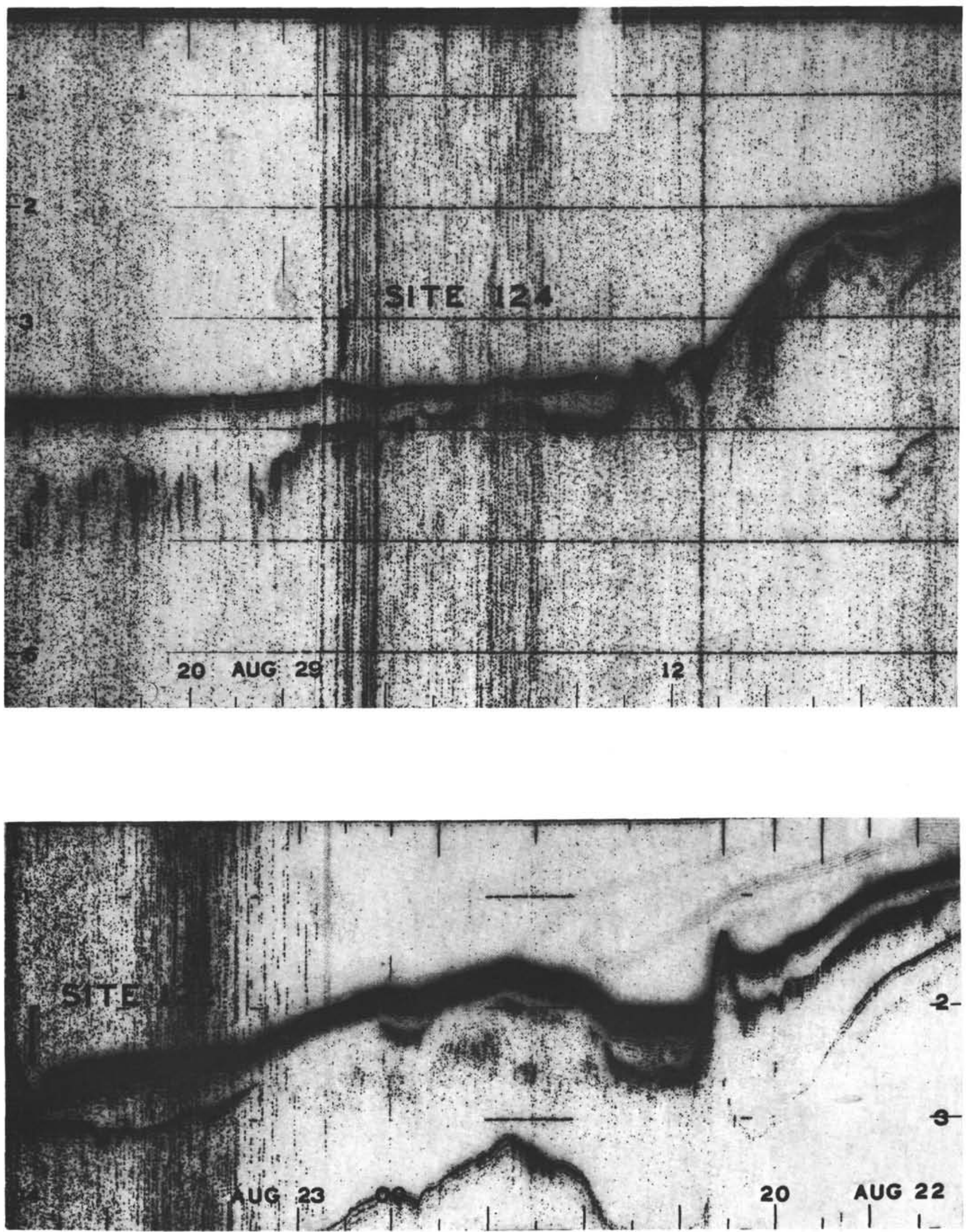

Figure 3S. 

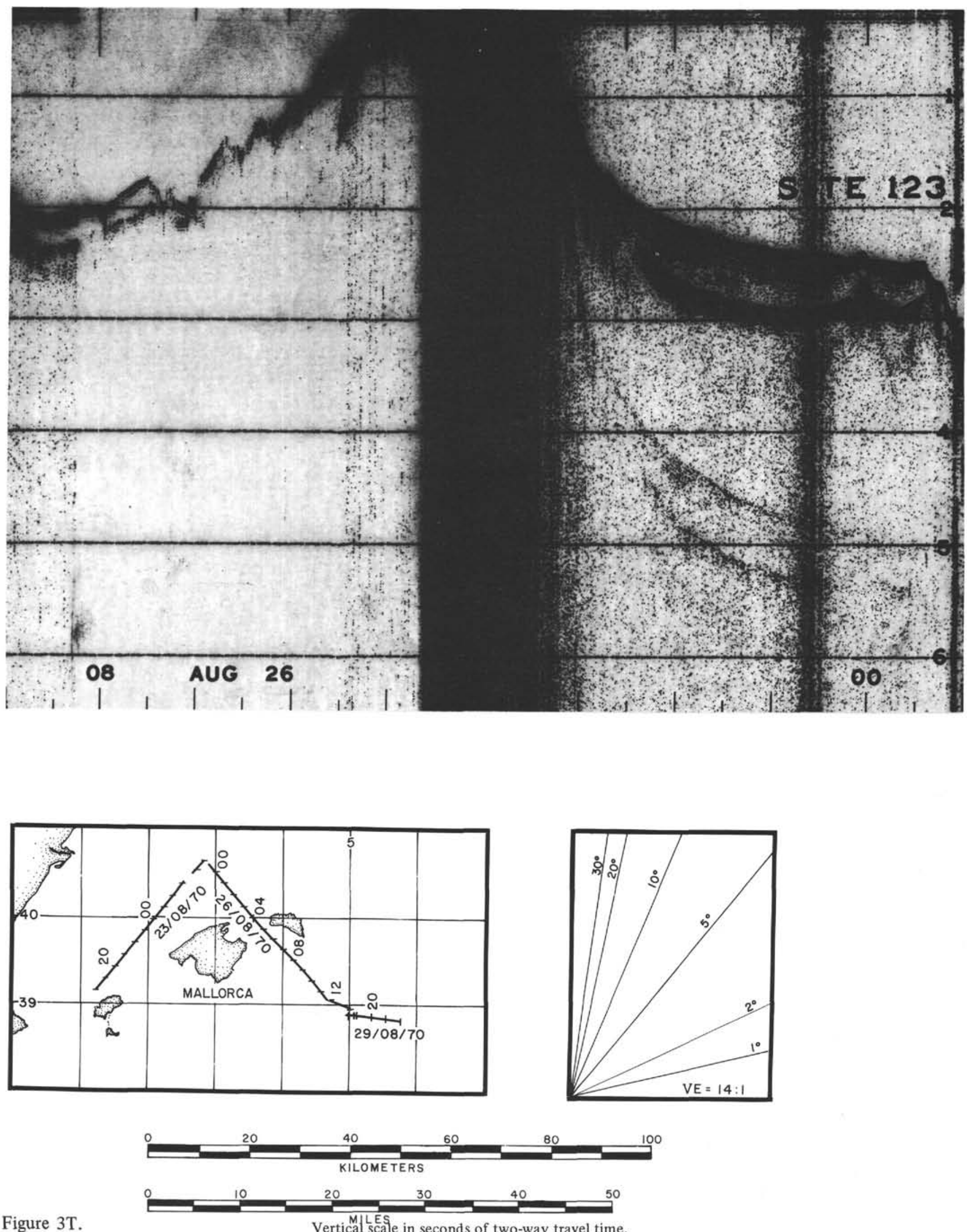

Figure 3T.

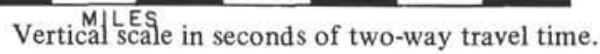


W. B. F. RYAN, T. B. GUSTAFSON
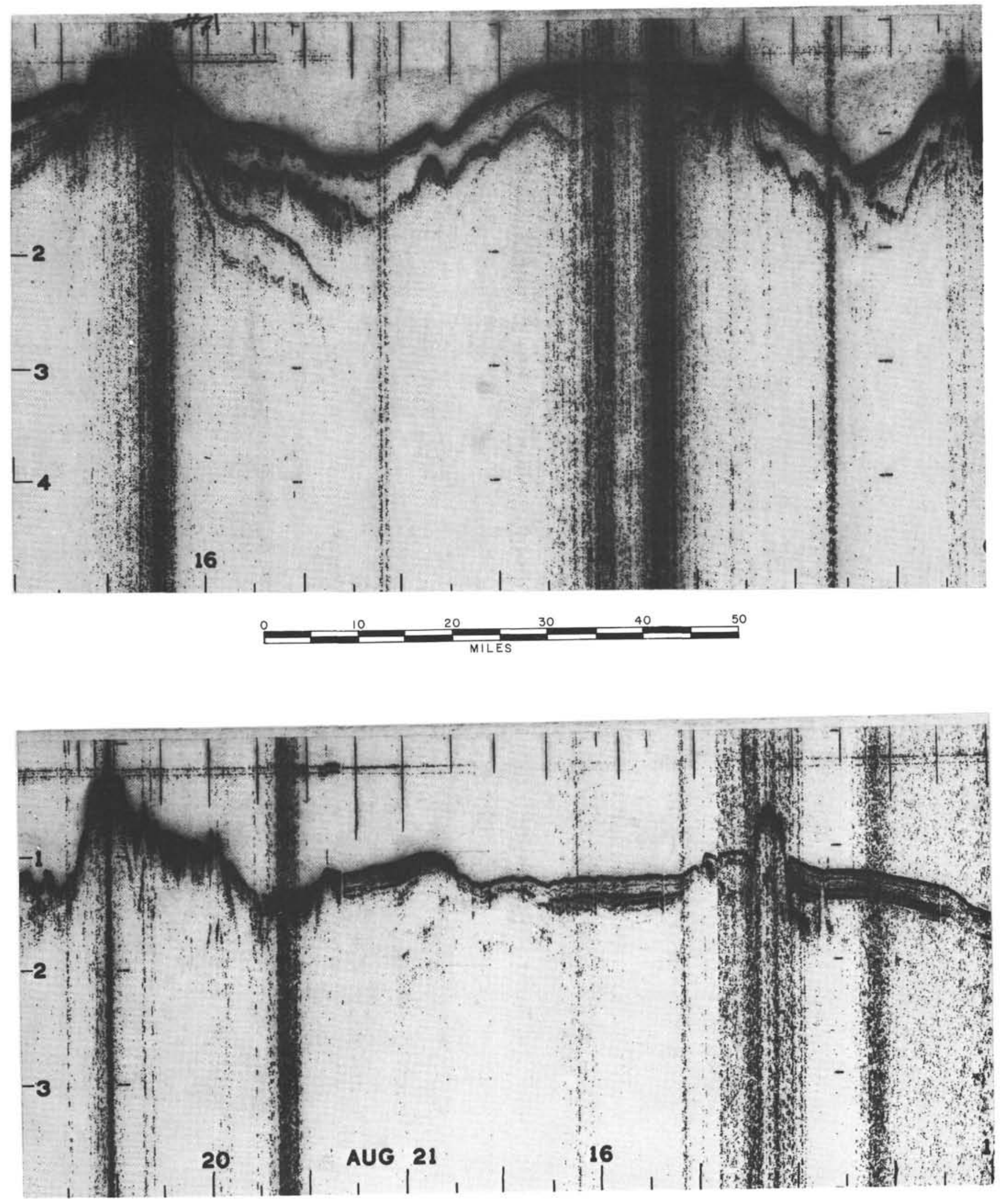

Figure 3U. 

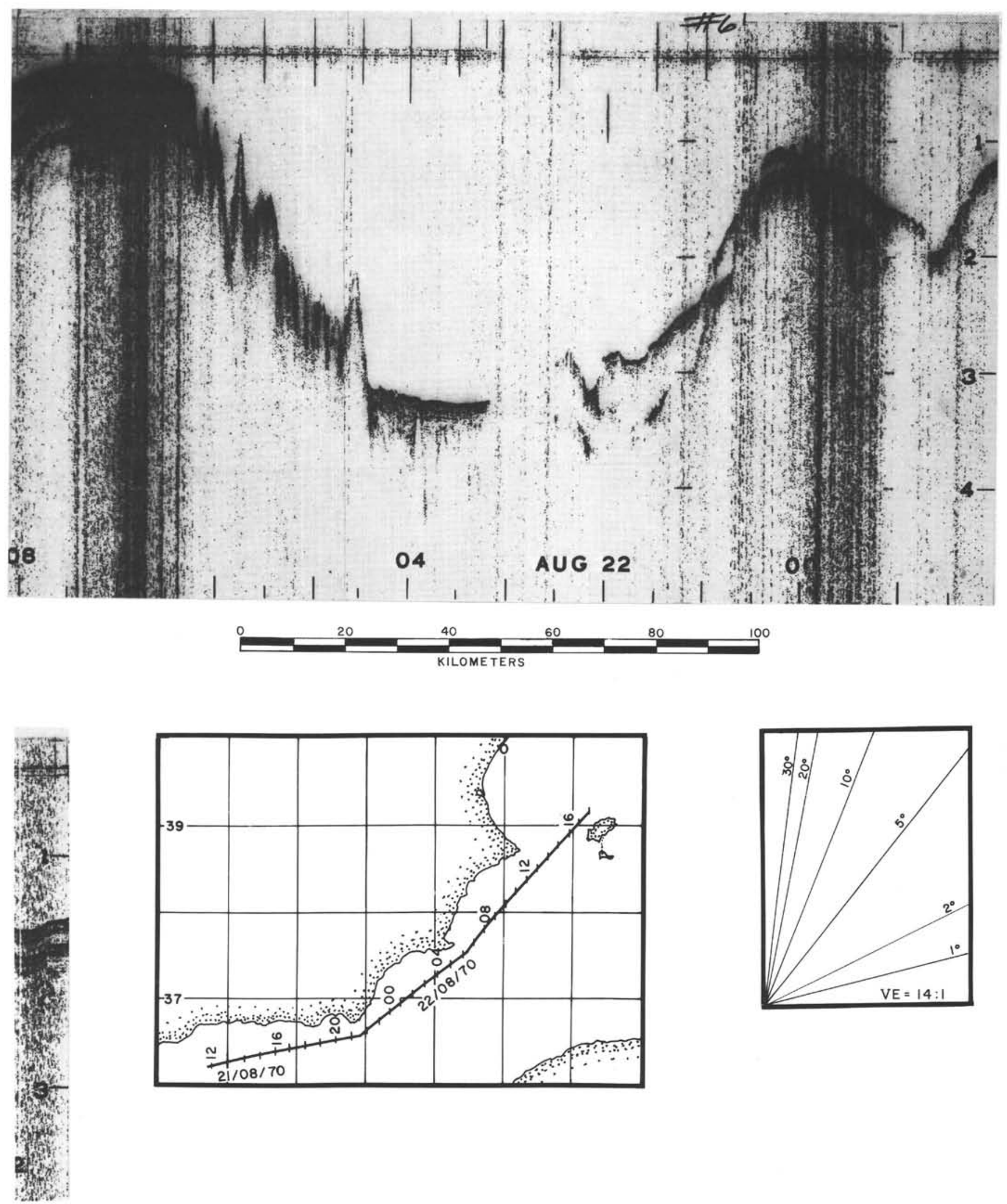

Figure 3V.

Vertical scale in seconds of two-way travel time. 

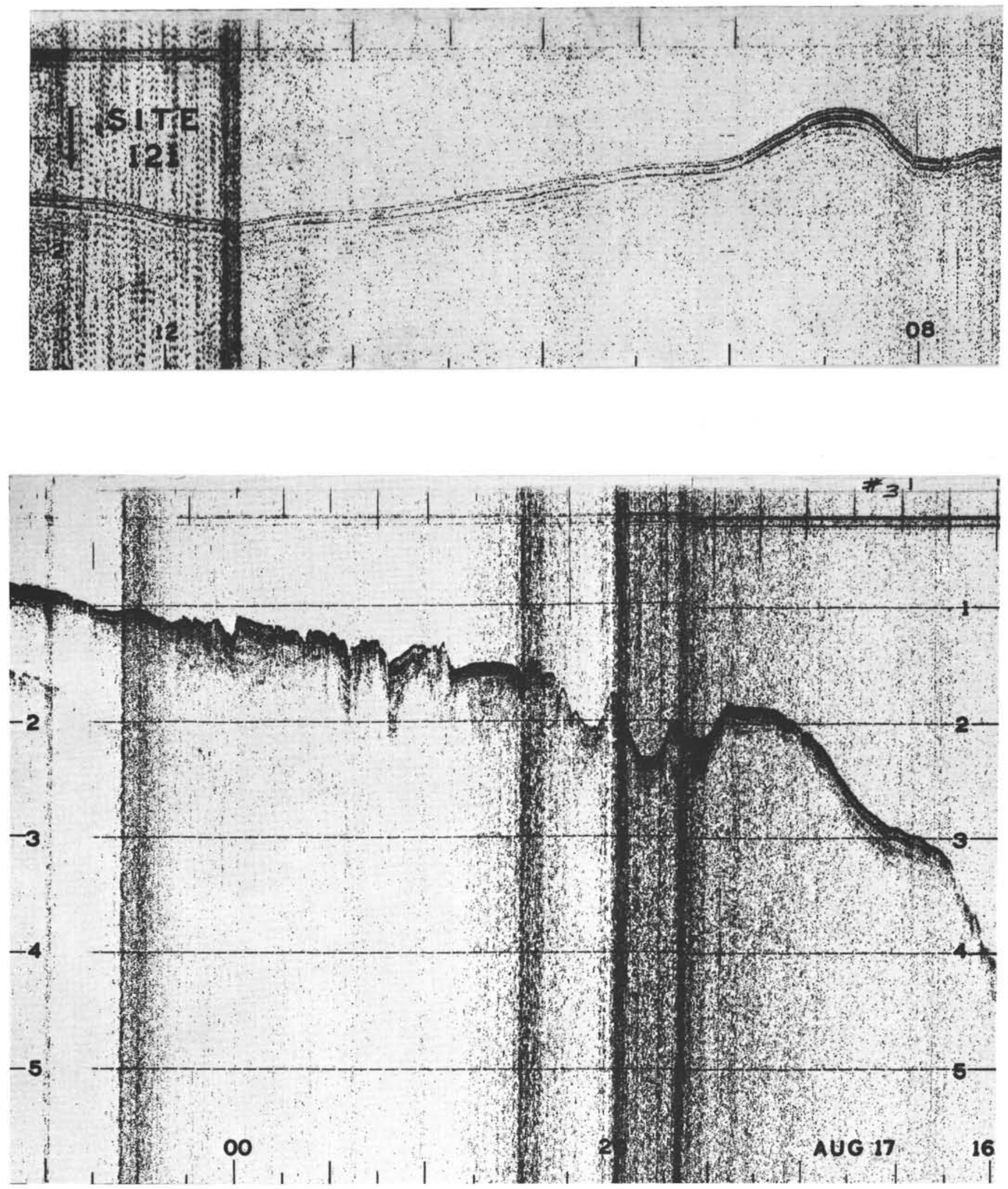

Figure $3 \mathrm{~W}$. 

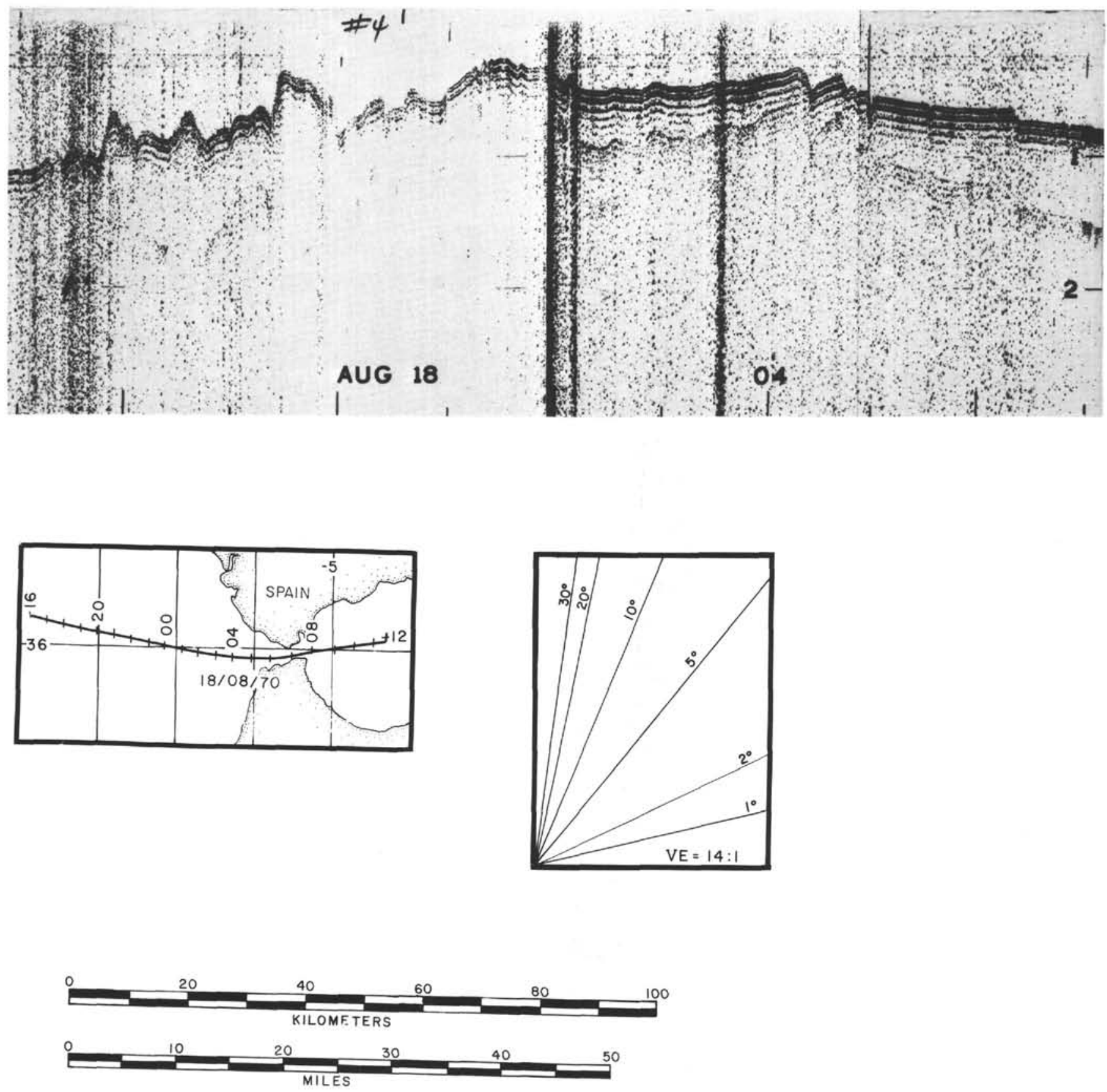

Figure $3 \mathrm{X}$.

Vertical scale in seconds of two-way travel time. 


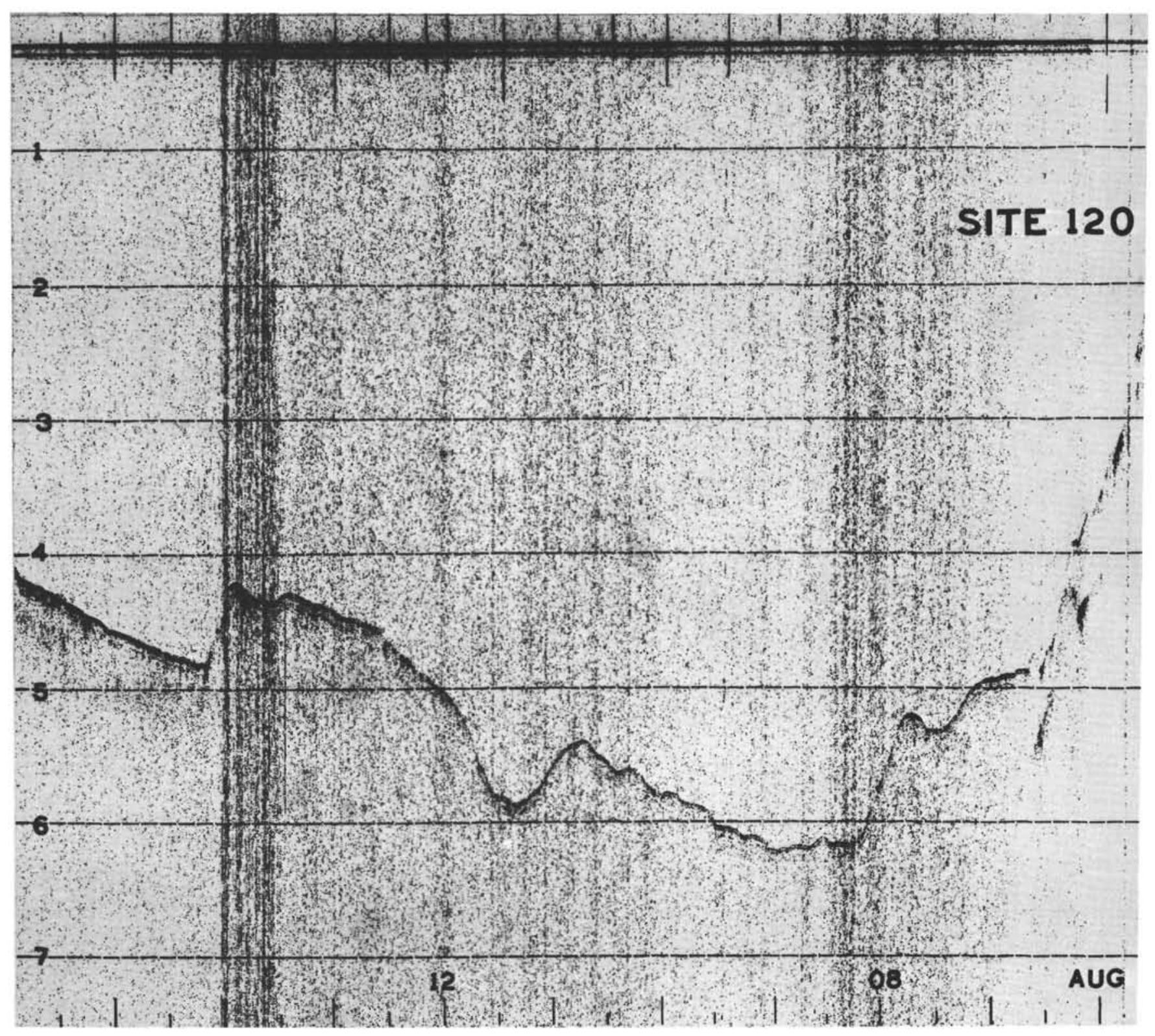

Figure $3 Y$. 
15. UNDERWAY GEOPHYSICAL MEASUREMENTS, EASTERN NORTH ATLANTIC AND MEDITERRANEAN

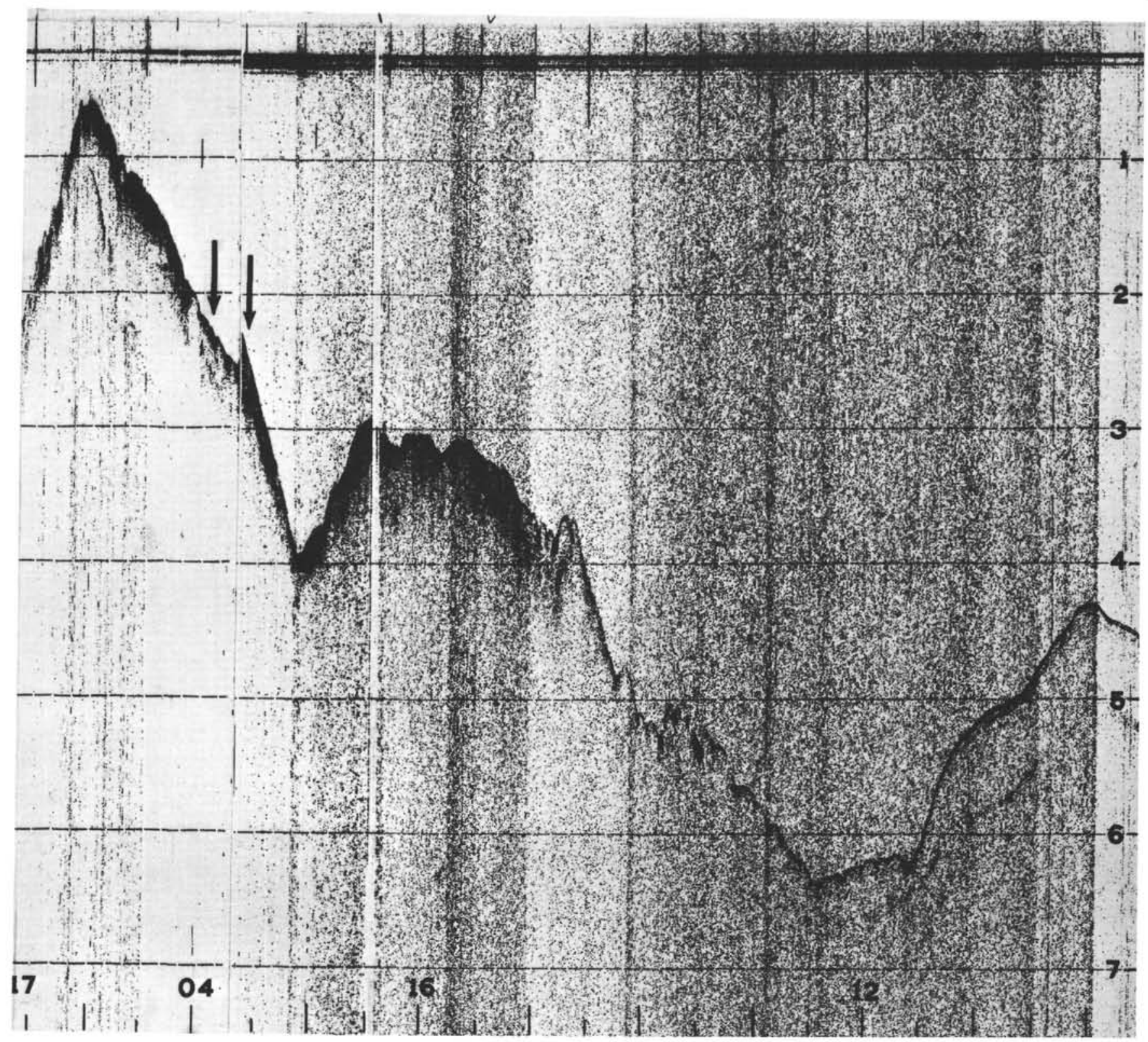

Figure 3Z.

Vertical scale in seconds of two-way travel time. 

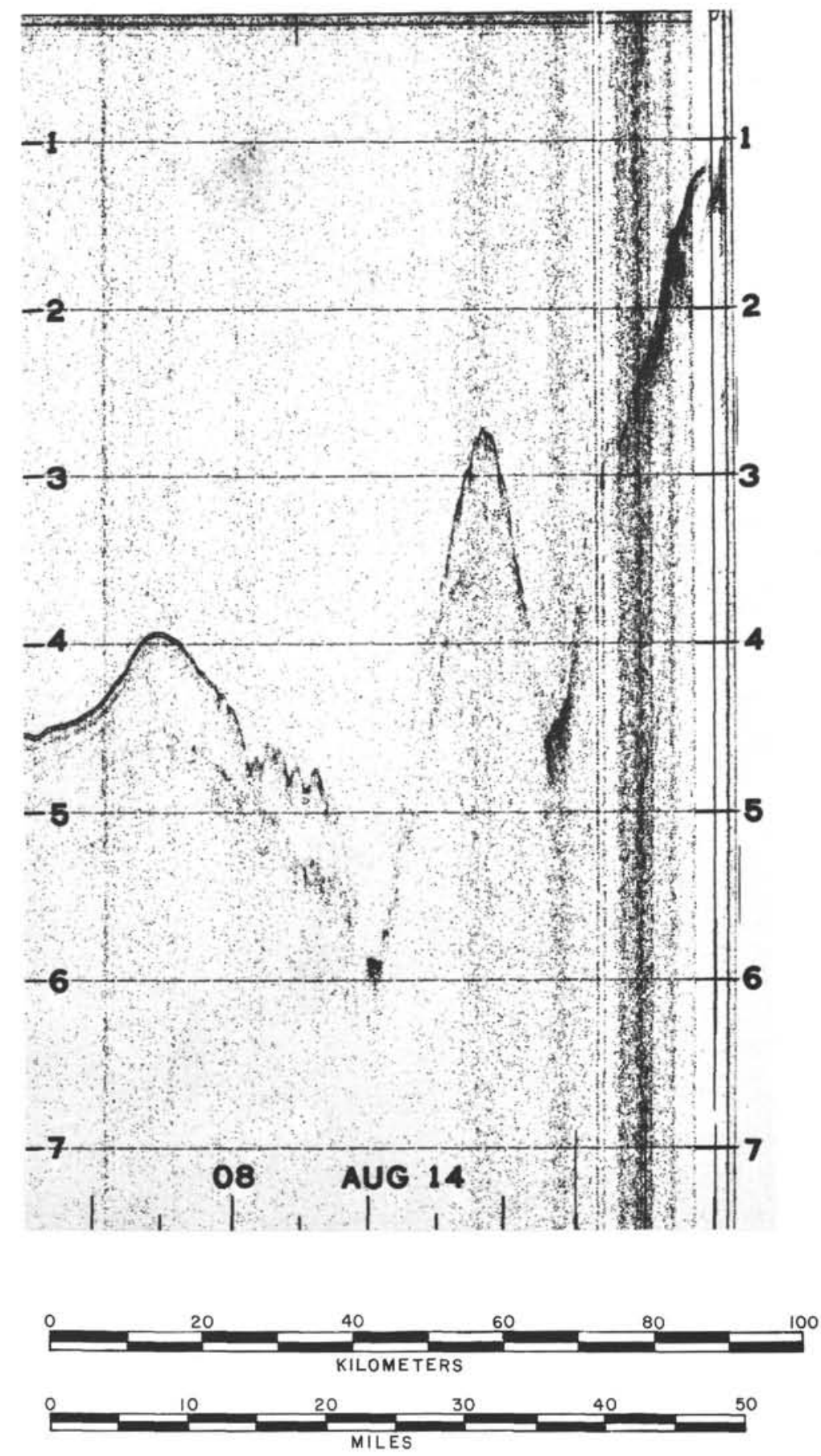

Figure 3AA.
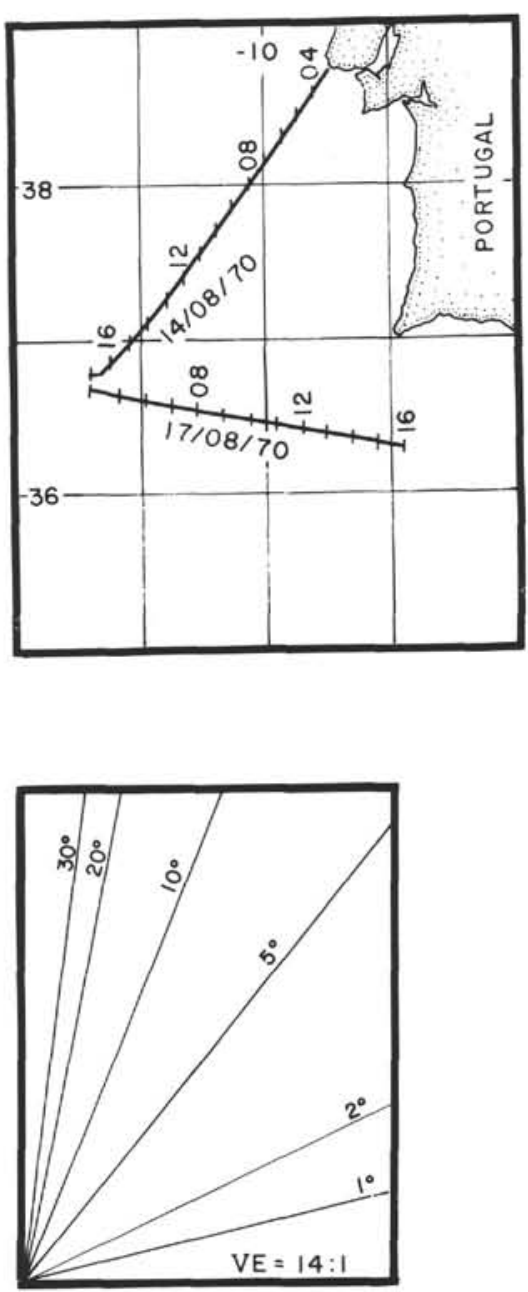